\title{
EXPLICIT BIRATIONAL GEOMETRY OF 3-FOLDS AND 4-FOLDS OF GENERAL TYPE, III
}

\author{
JUNGKAI A. CHEN AND MENG CHEN
}

\begin{abstract}
Nonsingular projective 3-folds $V$ of general type can be naturally classified into 18 families according to the pluricanonical section index $\delta(V):=\min \left\{m \mid P_{m} \geq 2\right\}$ since $1 \leq \delta(V) \leq 18$ due to our previous series (I, II). Based on our further classification to 3 -folds with $\delta(V) \geq 13$ and an intensive geometrical investigation to those with $\delta(V) \leq 12$, we prove that $\operatorname{Vol}(V) \geq \frac{1}{1680}$ and that the pluricanonical map $\Phi_{m}$ is birational for all $m \geq 61$, which greatly improves known results. An optimal birationality of $\Phi_{m}$ for the case $\delta(V)=2$ is obtained. As an effective application, we study projective 4 -folds of general type with $p_{g} \geq 2$ in the last section.
\end{abstract}

\section{Introduction}

One of the fundamental aspects of birational geometry is to understand the behavior of the natural pluricanonical map $\Phi_{m}$ of any variety for any $m \in \mathbb{Z}_{>0}$. The induced fibrations possibly reduce the studies to lower dimensional situations. Varieties of general type, which are those with birational pluricanonical maps $\Phi_{m}$ for sufficiently large $m$, are therefore considered as the basic building blocks of varieties.

For varieties of general type, a key problem is to find an effective integer $m>0$ so that $\Phi_{m}$ is birational. The remarkable theorem of Hacon and McKernan [17], Takayama [31, and Tsuji [32] says that there is a constant $c(n)$ so that $\Phi_{m}$ is birational for all $n$-dimensional varieties of general type and for all $m \geq c(n)$. However, these constants are explicitly known only when $n \leq 3$.

In fact, the problem is almost equivalent to find a practical lower bound of the canonical volume which computes the rate of growth of plurigenera, or equivalent to find $m_{0}$ such that plurigenus $P_{m_{0}}$ is sufficiently large. One may also refer to the nice survey article HaconMcKernan [18] for various boundedness results in birational geometry.

The motivation of this series is to study birational geometry of 3-folds and higher dimensional varieties of general type. The main purpose is to investigate the following:

The first author was partially supported by NCTS/TPE and National Science Council of Taiwan. The second author was supported by National Natural Science Foundation of China (\#11171068, \#11121101, \#11231003) and Doctoral Fund of Ministry of Education of China (\#20110071110003). 
Open problem 1.1. Find optimal constants $v_{3} \in \mathbb{Q}_{>0}$ and $b_{3} \in \mathbb{Z}_{>0}$ so that, for all nonsingular projective 3-folds $V$ of general type,

i. $\operatorname{Vol}(V) \geq v_{3}$ and

ii. $\Phi_{m}$ is birational for all $m \geq b_{3}$.

Recall that we have proved the following:

Theorem 1.2. (77, Theorems 1.1, 1.2]) Let $V$ be a nonsingular projective 3 -fold of general type. Then

1. $\operatorname{Vol}(V) \geq \frac{1}{2660}$;

2. there exists a positive integer $m_{0}(V) \leq 18$ so that $P_{m_{0}} \geq 2$;

3. the pluricanonical map $\Phi_{m}$ is birational onto its image for all $m \geq 73$.

For more results on explicit birational geometry of 3-folds of general type, one may refer to our previous papers [6, 7].

In order to formulate our main statements of this article, we need to recall some general results and introduce some definition. Given a projective variety $V$ of general type, there exists a minimal model $X$ birational to $V$ (cf. [2]). Thanks to the Riemann-Roch formula and Vanishing Theorem, $\operatorname{Vol}(V)=K_{X}^{\operatorname{dim} X}$. Notice that in dimension three or higher, a minimal model may have singularities. Hence $K_{X}^{\operatorname{dim} X}$ is just a positive rational number.

A minimal model has at worst terminal singularities. In dimension three, terminal singularities was classified by Mori. A three dimensional terminal singularity is one of the following: a terminal quotient singularity of type $\frac{1}{r}(1,-1, b)$ for some $b$ relatively prime to $r$ which we usually denote it as $(b, r)$ for short, an isolated cDV point, a quotient of an isolated cDV point. It is well-known to experts that a three dimensional terminal point can be deformed into a collection of terminal quotient singularities, which is called basket of singularities. An important feature of three dimensional birational geometry is the Singular Riemann-Roch formula due to Reid [29]

$$
\chi\left(X, m K_{X}\right)=\frac{m(m-1)(2 m-1) K_{X}^{3}}{12}+(1-2 m) \chi\left(X, \mathcal{O}_{X}\right)+l_{m},
$$

where $l_{m}$ denotes the contribution of singularities which can be computed by baskets. It follows that all plurigenera and hence canonical volume of a minimal 3 -fold $X$ are completely determined by $P_{2}(X)$, $\chi\left(X, \mathcal{O}_{X}\right)$ and baskets of singularities $B_{X}$, of which we called such a triple the weighted basket of $X$. For the basic properties of weighted baskets, one may refer to [6, Section 3]. Since our problems are birational in nature, the studies of nonsingular threefold $V$ is equivalent to the studies of its minimal model $X$. In particular, we may and do 
consider the weighted basket of $V$ as the weighted basket of its minimal model $X$. 1

Next, we would like to define the pluricanonical section index (or, in short, the ps-index)

$$
\delta(V):=\min \left\{m \mid m \in \mathbb{Z}_{>0}, P_{m}(V) \geq 2\right\},
$$

which is clearly a birational invariant. By Theorem 1.2, we have $\delta(V) \leq$ 18 for any 3 -fold $V$ of general type. Note that 3-folds $V$ with $\delta(V)=1$ (i.e., $p_{g}(V) \geq 2$ ) have been intensively studied in [10, 11] where optimal results are realized. Threefolds of general type with $\delta(V) \geq 2$ are far from being clear. Sometimes we use the symbol $\delta(X)$ directly since $X$ is birationally equivalent to $V$.

Example 1.3. The "worst" known minimal 3 -fold is the weighted hyper-surface $X:=X_{46} \subset \mathbb{P}(4,5,6,7,23)$ (cf. [15]) which has the invariants: $\delta(X)=10$ and $\operatorname{Vol}(X)=K_{X}^{3}=\frac{1}{420}$. Also $\Phi_{26}$ is not birational.

In this paper, we mainly investigate projective 3 -folds of general type with $\delta(V) \geq 2$. Our main results are as follows.

Theorem 1.4. (=Theorem 5.1) Let $V$ be a nonsingular projective 3fold of general type with $\delta(V) \geq 13$. Then its weighted basket $\mathbb{B}=$ $\left\{B_{V}, P_{2}(V), \chi\left(\mathcal{O}_{V}\right)\right\}$ belongs to one of the types in Tables $\mathrm{F}-0, \mathrm{~F}-1$, $\mathrm{F}-2$ in Appendix and the following is true:

(1) $\delta(V)=18$ if and only if $\mathbb{B}(V)=\left\{B_{2 a}, 0,2\right\}$;

(2) $\delta(V) \neq 16,17$

(3) $\delta(V)=15$ if and only if $\mathbb{B}(V)$ belongs to one of the types in Table $\mathrm{F}-1$;

(4) $\delta(V)=14$ if and only if $\mathbb{B}(V)$ belongs to one of the types in Table $\mathrm{F}-2$;

(5) $\delta(V)=13$ if and only if $\mathbb{B}(V)=\left\{B_{41}, 0,2\right\}$,

where $B_{2 a}$ and $B_{41}$ can be found in Table $\mathrm{F}-0$

Some other results for 3-folds with large $\delta(V)$ are given in Section 4. For example, one has

Corollary 1.5. (=Corollary 4.8) Let $V$ be a nonsingular projective 3-fold of general type with $\operatorname{Vol}(V)<\frac{1}{336}$. Then $\delta(V) \geq 8$.

We also prove the following:

Theorem 1.6. Let $V$ be a nonsingular projective 3-fold of general type. Then

(1) $\Phi_{m}$ is birational for all $m \geq 61$;

\footnotetext{
${ }^{1}$ Even though minimal models are not necessarily unique, it is known that two birational minimal models are connected by flops (cf. [21]). Together with the fact that a 3-dimensional flop preserves singularity types (cf. 23]), it follows that baskets of $V$ is independent of choices of minimal models.
} 
(2) $\operatorname{Vol}(V) \geq \frac{1}{1680}$. Furthermore, $\operatorname{Vol}(V)=\frac{1}{1680}$ if and only if $\mathbb{B}(V)=\left\{B_{7 a}, 0,2\right\}$ or $\left\{B_{36 a}, 0,2\right\}$, where $B_{7 a}$ and $B_{36 a}$ can be found in Table $\mathrm{F}-2$

A direct by-product of our method is the following:

Corollary 1.7. Let $V$ be a nonsingular projective 3-fold of general type with $p_{g}(V)=1$. Then

(1) $\operatorname{Vol}(V) \geq \frac{1}{75}$;

(2) $\Phi_{m}$ is birational for all $m \geq 18$.

In the second part of this paper we prove some optimal results on 3 -folds with $\delta(V)=2$.

Theorem 1.8. Let $V$ be a nonsingular projective 3-fold of general type with $\delta(V) \leq 2$. Then

(1) $\Phi_{m}$ is birational for all $m \geq 11$;

(2) If $\Phi_{10}$ is not birational, then $0 \leq \chi\left(\mathcal{O}_{V}\right) \leq 3$ and $\left|2 K_{V}\right|$ is composed of a rational pencil of $(1,2)$ surfaces. Furthermore, $\#\{\mathbb{B}(V)\}<+\infty$ and the initial basket $B^{0}$ of $B_{V}$ belongs to one of the types in Tables II-1, II-2, II-3 in the Appendix.

The following examples show that our results in Theorem 1.8 are optimal.

Example 1.9. (Iano-Fletcher [15, P. 151, P. 153])

(1) General weighted complete intersections $X_{22} \subset \mathbb{P}(1,2,3,4,11)$ and $X_{6,18} \subset \mathbb{P}(2,2,3,3,4,9)$ both have ps-index $\delta=2$. Since both $X_{22}$ and $X_{6,18}$ have non-birational 10-canonical map, Theorem 1.8(1) is optimal.

(2) The 3 -fold $X_{22}$ corresponds to No. 1 in Table II- 1 with $\chi=0$ and $X_{6,18}$ belongs to No. 11 (with $t=1$ ) in Table II-1.

Remark 1.10. Theorem [1.8 is parallel to main results in [10]. We have similar statements to Theorem 1.8 for 3 -folds with $\delta(V) \geq 3$. We omit them since we are not sure whether they are optimal or not.

In the last part we study projective 4 -folds. The main result is the following:

Theorem 1.11. (=Theorem 8.2) Let $V$ be a nonsingular projective 4-fold of general type. Then,

(i) when $p_{g}(V) \geq 2, \Phi_{\left|m K_{V}\right|}$ is birational for all $m \geq 35$;

(ii) when $p_{g}(V) \geq 19, \Phi_{\left|m K_{V}\right|}$ is birational for all $m \geq 18$.

This paper is organized as follows. In Section 2, we start with general setting on rational maps on varieties of general type and review some known useful inequalities. Then we list several basic lemmas on 3folds. In Section 3, we improve our technique used in [7] to bound $K_{X}^{3}$ 
from below. Applying our basket analysis developed in [6], we obtain an effective function $v(x)$ in Section 4 so that $K_{X}^{3} \geq v(\delta(X))$ for any given minimal 3 -fold $X$. Section 5 is devoted to compiling the clean list for $\mathbb{B}(X)$ with $\delta(X) \geq 13$. Then, in Section 6 , we are able to study the birationality of $\Phi_{m}$. Section 7 is dedicated to classifying 3-folds with $\delta=2$. Finally we study nonsingular projective 4 -folds of general type with $p_{g} \geq 2$ in Section 8. All subsidiary tables are presented in the Appendix.

Throughout we work over any algebraically closed field $k$ of characteristic 0 . We are in favor of the following symbols:

○ " " denotes linear equivalence or $\mathbb{Q}$-linear equivalence;

○ "三” denotes numerical equivalence;

○ " $|A| \preceq|B|$ " means that $|B| \supseteq|A|+$ fixed effective divisors.

\section{Preliminaries}

We begin with the general setting on rational maps defined by some sub-linear system of the pluricanonical system $|m K|$ on varieties of general type. Let $V$ be any nonsingular projective variety of general type with dimension $n \geq 3$. According to the Minimal Model Program, $V$ has a minimal model (see e.g. [22, [24], 2] and [30]). From the point of view of birational geometry, we may always consider the rational map on minimal varieties of general type. A minimal model $X$ is a normal projective variety with a nef canonical divisor $K_{X}$ and with $\mathbb{Q}$-factorial terminal singularities.

2.1. The rational map $\Phi_{\Lambda}$ for $\Lambda \subset\left|m_{0} K\right|$. Let $X$ be a minimal projective variety of general type on which $P_{m_{0}}(X) \geq 2$ for a positive integer $m_{0}$. Let $\Lambda \subset\left|m_{0} K_{X}\right|$ be a positive dimensional linear system. Fix an effective Weil divisor $K_{m_{0}} \sim m_{0} K_{X}$ on $X$. Take successive blow-ups $\pi: X^{\prime} \rightarrow X$ along nonsingular centers, such that the following conditions are satisfied:

(i) $X^{\prime}$ is smooth;

(ii) the moving part of $\pi^{*}(\Lambda)$ is base point free and so that $g:=$ $\Phi_{\Lambda} \circ \pi$ is a non-constant morphism;

(iii) $\pi^{*}\left(K_{m_{0}}\right) \cup\{\pi$-exceptional divisors $\}$ has simple normal crossing supports.

Sometimes we will take further blow-ups so that $\pi$ satisfies some more conditions, which will be specified explicitly.

We have a morphism $g: X^{\prime} \longrightarrow \overline{\Phi_{\Lambda}(X)} \subseteq \mathbb{P}^{N}$. Let $X^{\prime} \stackrel{f}{\longrightarrow} \Gamma \stackrel{s}{\longrightarrow}$ $\overline{\Phi_{\Lambda}(X)}$ be the Stein factorization of $g$. We have the following commutative diagram: 


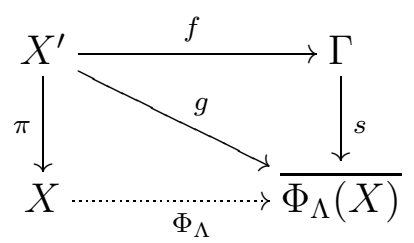

We may write $m_{0} K_{X^{\prime}}={ }_{\mathbb{Q}} \pi^{*}\left(m_{0} K_{X}\right)+E_{\pi, m_{0}}$ where $E_{\pi, m_{0}}$ is an effective $\pi$-exceptional $\mathbb{Q}$-divisor. Denote by $M_{m_{0}}$ (resp. $\left.M_{\Lambda}\right)$ the movable part of $\left|m_{0} K_{X^{\prime}}\right|$ (resp. $\pi^{*} \Lambda$ ). Set $d_{m_{0}}:=\operatorname{dim} \Phi_{m_{0}}(X)$ (resp. $\left.d_{\Lambda}:=\operatorname{dim} \Gamma\right)$. The Bertini Theorem implies that the general member of the moving part $M_{\Lambda}$ of $\pi^{*}(\Lambda)$ is irreducible whenever $d_{\Lambda} \geq 2$ and, otherwise, $M_{\Lambda} \equiv a_{\Lambda} F$, where $a_{\Lambda}:=\operatorname{deg} f_{*} \mathcal{O}_{X^{\prime}}\left(M_{\Lambda}\right)$ and $F$ is a general fiber of $f$. We set

$$
\theta_{\Lambda}:= \begin{cases}1, & \text { if } d_{\Lambda} \geq 2 \\ a_{\Lambda}, & \text { if } d_{\Lambda}=1\end{cases}
$$

Recall our definition in [7, Definition 2.4], the generic irreducible element $\Sigma$ of $\pi^{*}(\Lambda)$ is defined as follows:

$\Sigma_{\Lambda}:= \begin{cases}\text { the general member of the moving part of } \pi^{*}(\Lambda), & \text { if } d_{\Lambda} \geq 2 \\ F, & \text { if } d_{\Lambda}=1\end{cases}$

By the above setting, we always have

$$
m_{0} \pi^{*}\left(K_{X}\right) \sim_{\mathbb{Q}} \theta_{\Lambda} \Sigma_{\Lambda}+E_{\Lambda}^{\prime}
$$

for some effective $\mathbb{Q}$-divisor $E_{\Lambda}^{\prime}$ on $X^{\prime}$.

Convention. Whenever we are working on the complete linear system $\left|m_{0} K_{X}\right|$, we will use parallel notations such as $d_{m_{0}}, \theta_{m_{0}}, \cdots$ (or even just $d, \theta, \cdots$, for simplicity).

We discuss the special case with $d_{\Lambda}=1$. Clearly the general fiber $F$ is nonsingular projective of $\operatorname{dimension} \operatorname{dim}(X)-1$. Replace $X^{\prime}$ by its birational model, we may assume that there is a birational contraction morphism $\sigma: F \longrightarrow F_{0}$ onto a minimal model $F_{0}$. We have the following "canonical restriction inequality":

Lemma 2.1. Keep the above settings. Suppose that $d_{\Lambda}=1$. The following holds:

(i) if $b:=g(\Gamma)>0$, then $\left.\pi^{*}\left(K_{X}\right)\right|_{F} \sim \sigma^{*}\left(K_{F_{0}}\right)$;

(ii) if $b=0$, then

$$
\left.\pi^{*}\left(K_{X}\right)\right|_{F} \geq \frac{\theta_{\Lambda}}{m_{0}+\theta_{\Lambda}} \sigma^{*}\left(K_{F_{0}}\right) .
$$

Proof. Statement (i) follows from Chen [14, Lemma 2.5]. 
Assume $\Gamma \cong \mathbb{P}^{1}$. Choose a sufficiently large and divisible integer $m$ so that both $\left|m \pi^{*}\left(K_{X}\right)\right|$ and $\left|m K_{F_{0}}\right|$ are base point free. By Kawamata's extension theorem [20, Theorem A], we have the surjective map:

$$
H^{0}\left(X^{\prime}, m \theta_{\Lambda}\left(K_{X^{\prime}}+F\right)\right) \longrightarrow H^{0}\left(F, m \theta_{\Lambda} K_{F}\right) .
$$

Since $\left|m\left(\theta_{\Lambda}+m_{0}\right) K_{X^{\prime}}\right| \succeq\left|m \theta_{\Lambda}\left(K_{X^{\prime}}+F\right)\right|$, Mov $\left|m \theta_{\Lambda} K_{F}\right|=\left|m \theta_{\Lambda} \sigma^{*}\left(K_{F_{0}}\right)\right|$ and $\left|m\left(\theta_{\Lambda}+m_{0}\right) \pi^{*}\left(K_{X}\right)\right|=\left|M_{m\left(\theta_{\Lambda}+m_{0}\right)}\right|$, we obtain the following inequality:

$$
\left.m\left(\theta_{\Lambda}+m_{0}\right) \pi^{*}\left(K_{X}\right)\right|_{F}=\left.M_{m\left(\theta_{\Lambda}+m_{0}\right)}\right|_{F} \geq m \theta_{\Lambda} \sigma^{*}\left(K_{F_{0}}\right),
$$

which implies (ii).

2.2. Key inequalities on 3-folds. Let $X$ be minimal 3 -fold of general type. Assume that $\Lambda \subset\left|m_{0} K_{X}\right|$ is a linear system of positive dimension. As in 2.1, we obtain an induced fibration $f: X^{\prime} \longrightarrow \Gamma$. Pick a generic irreducible element $S$ of $\left|m_{0} K_{X^{\prime}}\right|$. Let $|G|$ be a given base point free linear system on $S$. Pick a generic irreducible element $C$ of $|G|$. Since $\left.\pi^{*}\left(K_{X}\right)\right|_{S}$ is nef and big, Kodaira's lemma implies that $\left.\pi^{*}\left(K_{X}\right)\right|_{S} \geq \beta C$ for some rational number $\beta>0$. Then, by [7, Inequality $(2.1)]$, one has

$$
K_{X}^{3} \geq \frac{\theta \beta}{m_{0}} \xi
$$

where $\xi:=\left(\pi^{*}\left(K_{X}\right) \cdot C\right)_{X^{\prime}}$. Besides, by [7, Remark 2.12], one has

$$
\xi \geq \frac{\operatorname{deg}\left(K_{C}\right)}{1+\frac{m_{0}}{\theta}+\frac{1}{\beta}} .
$$

For any positive integer $m$ so that $\alpha_{m}:=\left(m-1-\frac{m_{0}}{\theta}-\frac{1}{\beta}\right) \xi>1$, by Chen-Zuo [13, Theorem 3.1], one has

$$
\xi \geq \frac{\operatorname{deg}\left(K_{C}\right)+\left\lceil\alpha_{m}\right\rceil}{m}
$$

We have a stronger form of Inequality (2.3) when $C$ is "even":

Lemma 2.2. Under the above situation, if $C$ is an even divisor on $S$ (i.e. $\left.\frac{1}{2} C \in \operatorname{Pic}(S)\right)$, then, for any $m>0$ so that $\alpha_{m}>0$, one has

$$
\xi \geq \frac{\operatorname{deg}\left(K_{C}\right)+2\left\lceil\frac{1}{2} \alpha_{m}\right\rceil}{m} .
$$

Proof. We refer to the proof for Chen-Zuo [13, Theorem 3.1]. The key point is to estimate $\operatorname{deg}(D)$ where $D=\left.\lceil Q\rceil\right|_{C}$ and $Q$ is a $\mathbb{Q}$-divisor on $S$ with $(Q \cdot C)=\alpha_{m}$. Since $\operatorname{deg}(D) \geq \alpha_{m}>0$ and $\operatorname{deg}(D)$ is even, we naturally have

$$
\operatorname{deg}(D)=2\left(\lceil Q\rceil \cdot \frac{1}{2} C\right) \geq 2\left\lceil\frac{1}{2} \alpha_{m}\right\rceil
$$

where we note that $\left(\lceil Q\rceil \cdot \frac{1}{2} C\right)$ is a positive integer. Clearly the rest of the proof of Chen-Zuo [13, Theorem 3.1] implies Inequality (2.4). 
When $d_{\Lambda}=1$, Lemma 2.1(ii) implies the following:

$$
\xi=\left(\pi^{*}\left(K_{X}\right) \cdot C\right)_{X^{\prime}} \geq \frac{\theta}{m_{0}+\theta}\left(\sigma^{*}\left(K_{F_{0}}\right) \cdot C\right)_{F} .
$$

\subsection{Other useful Lemmas.}

Lemma 2.3. (see Maşek [25, Proposition 4] or [12, Lemma 2.6]) Let $S$ be a nonsingular projective surface. Let $L$ be a nef and big $\mathbb{Q}$-divisor on $S$ satisfying the following conditions:

(1) $L^{2}>8$

(2) $\left(L \cdot C_{x}\right) \geq 4$ for all irreducible curves $C_{x}$ passing through any very general point $x \in S$.

Then the linear system $\left|K_{S}+\lceil L\rceil\right|$ separates two distinct points in very general positions. Consequently, $\left|K_{S}+\lceil L\rceil\right|$ gives a birational map.

Lemma 2.4. Let $\sigma: S \longrightarrow S_{0}$ be a birational contraction from a nonsingular projective surface $S$ of general type onto the minimal model $S_{0}$. Assume that $\left(K_{S_{0}}^{2}, p_{g}\left(S_{0}\right)\right) \neq(1,2)$ and that $C$ is a moving curve on $S$. Then $\left(\sigma^{*}\left(K_{S_{0}}\right) \cdot C\right) \geq 2$.

Proof. When $K_{S_{0}}^{2} \geq 2$, this is due to Hodge index theorem. When $\left(K_{S_{0}}^{2}, p_{g}\left(S_{0}\right)\right)=(1,0)$, this is due to Miyaoka [26, Lemma 5]. When $\left(K_{S_{0}}^{2}, p_{g}\left(S_{0}\right)\right)=(1,1),\left(\sigma^{*}\left(K_{S_{0}}\right) \cdot C\right)=1$ implies $K_{S_{0}} \equiv \sigma_{*} C$ by Hodge index theorem. According to Bombieri [3], we know that $S_{0}$ is simply connected. Thus $K_{S_{0}} \sim \sigma_{*} C$, which is impossible since $\left|K_{S_{0}}\right|$ is not movable.

Lemma 2.5. Let $\sigma: S \longrightarrow S_{0}$ be the birational contraction onto the minimal model $S_{0}$ from a nonsingular projective surface $S$ of general type. Assume that $\left(K_{S_{0}}^{2}, p_{g}\left(S_{0}\right)\right) \neq(1,2)$ and that $\tilde{C}$ is a curve on $S$ passing through very general points. Then $\left(\sigma^{*}\left(K_{S_{0}}\right) \cdot \tilde{C}\right) \geq 2$.

Proof. In fact, by the projection formula, this is equivalent to see $\left(K_{S_{0}}\right.$. $\left.C_{0}\right) \geq 2$ for any curve $C_{0} \subset S_{0}$ passing through very general points of $S_{0}$.

On the contrary, let us assume $\left(K_{S_{0}} \cdot C_{0}\right) \leq 1$. Then $g\left(C_{0}\right) \geq 2$ implies $C_{0}^{2} \geq 1$. The Hodge index theorem says $K_{S_{0}}^{2}=1$ and $K_{S_{0}} \equiv C_{0}$. Recall that $S_{0}$ is not a $(1,2)$ surface. So $S_{0}$ must be either a $(1,0)$ surface or a $(1,1)$ surface.

If $\left(K_{S_{0}}^{2}, p_{g}\left(S_{0}\right)\right)=(1,0)$, then $q\left(S_{0}\right)=0$ and the torsion element $\theta:=$ $K_{S_{0}}-C_{0}$ is of order $\leq 5$ (see Reid [27]) and $h^{0}\left(S_{0}, C_{0}\right)=1$. Thus there are at most finite number of such curves on $S_{0}$ since \#Tor $\left(S_{0}\right) \leq 5$, which is absurd by the choice of $C_{0}$.

If $\left(K_{S_{0}}^{2}, p_{g}\left(S_{0}\right)\right)=(1,1)$, then $q\left(S_{0}\right)=0$ and $K_{S_{0}} \sim C_{0}$ since $\operatorname{Tor}\left(S_{0}\right)=$ 0 by Bombieri [3, Theorem 15] and thus $C_{0}$ is the unique canonical curve of $S_{0}$, which is absurd as well. 


\subsection{The birationality principle.}

Definition 2.6. Pick two different generic irreducible elements $S^{\prime}, S^{\prime \prime}$ (resp. $C^{\prime}, C^{\prime \prime}$ ) in $\left|M_{m_{0}}\right|$ (resp. in $|G|$ ).

(1) We say that $\left|m K_{X^{\prime}}\right|$ distinguishes $S^{\prime}$ and $S^{\prime \prime}$ if $\Phi_{\left|m K_{X^{\prime}}\right|}\left(S^{\prime}\right) \neq$ $\Phi_{\left|m K_{X^{\prime}}\right|}\left(S^{\prime \prime}\right)$.

(2) We say that $\left|m K_{X^{\prime}}\right|$ distinguishes $C^{\prime}$ and $C^{\prime \prime}$ if $\Phi_{\left|m K_{X^{\prime}}\right|}\left(C^{\prime}\right) \neq$ $\Phi_{\left|m K_{X^{\prime}}\right|}\left(C^{\prime \prime}\right)$.

We will apply the useful, but technical theorem in Chen-Zuo [13] for the birationality of $\Phi_{m}$.

Theorem 2.7. (see Chen-Zuo [13, Theorem 3.1] or [7, Theorem 2.11, Part 2]) Keep the same notations as above. Assume that, for some $m>0,\left|m K_{X^{\prime}}\right|$ distinguishes $S^{\prime}$ and $S^{\prime \prime}, C^{\prime}$ and $C^{\prime \prime}$ for generic $S^{\prime} \neq S^{\prime \prime}$, $C^{\prime} \neq C^{\prime \prime}$. Then $\Phi_{m}$ is birational under one of the following conditions:

(i) $\alpha_{m}>2$;

(ii) $\alpha_{m}>1$ and $C$ is not hyper-elliptic.

\section{The lower bound of $K^{3}$ in terms of $m_{0}$}

In the study of 3-dimensional explicit birational geometry, a challenging problem is to determine whether a given weighted basket $\mathbb{B}$ is geometric, i.e. equal to $\mathbb{B}_{X}$ for some 3 -fold $X$ or not. By exploiting geometric properties, one might be able to have a better estimation of the lower bound of $K_{X}^{3}$, and hence exclude some non-geometric formal baskets. In fact, in [7, 2.19 2.31], we already proved some effective inequalities for $K_{X}^{3}$. We shall go further along this direction in this section

Let $X$ be a minimal 3 -fold of general type. Assume $P_{m_{0}}(X) \geq 2$. Mostly we will take $\Lambda=\left|m_{0} K_{X}\right|$. Keep the settings in 2.1 and 2.2.

3.1. The case $d_{m_{0}}=3$.

If we take $|G|$ to be $|S|_{S} \mid$, then $\beta=\frac{1}{m_{0}}$. It is known, from [7, 2.19], that $\operatorname{deg}\left(K_{C}\right) \geq 6, \xi \geq \frac{10}{3 m_{0}+2}$ and $K_{X}^{3} \geq \frac{\xi}{m_{0}^{2}}$. Take $m=5 m_{0}+4, \cdots,(2 t+$ 1) $m_{0}+2 t$, successively. Then, by (2.3), one has $\xi \geq \frac{17}{5 m_{0}+4}, \frac{24}{7 m_{0}+6}$, $\cdots, \frac{7 t+3}{(2 t+1) m_{0}+2 t}$ respectively. Taking the limit, we obtain $\xi \geq \frac{7}{2 m_{0}+2}$. Therefore

$$
K_{X}^{3} \geq \frac{7}{2 m_{0}^{2}\left(m_{0}+1\right)} .
$$

In fact, for each small $m_{0}$, the explicit lower bound of $K^{3}$ can be slightly improved by the same trick and here is the result: 


\begin{tabular}{|c|c|c|c|c|c|c|c|}
\hline$m_{0}=$ & 2 & 3 & 4 & 5 & 6 & 7 & 8 \\
\hline$\xi \geq$ & $4 / 3$ & 1 & $3 / 4$ & $5 / 8$ & $1 / 2$ & $6 / 13$ & $2 / 5$ \\
\hline$K^{3} \geq$ & $1 / 3$ & $1 / 9$ & $3 / 64$ & $1 / 40$ & $1 / 72$ & $6 / 637$ & $1 / 160$ \\
\hline \hline$m_{0}=$ & 9 & 10 & 11 & 12 & 13 & 14 & 15 \\
\hline$\xi \geq$ & $4 / 11$ & $1 / 3$ & $3 / 10$ & $5 / 18$ & $1 / 4$ & $6 / 25$ & $2 / 9$ \\
\hline$K^{3} \geq$ & $4 / 891$ & $1 / 300$ & $3 / 1210$ & $5 / 2592$ & $1 / 696$ & $3 / 2450$ & $2 / 2025$ \\
\hline
\end{tabular}

3.2. The case $d_{m_{0}}=2$.

If we take $|G|=|S|_{S} \mid$, then $\beta \geq \frac{P_{m_{0}}-2}{m_{0}}$. By Inequality (2.3), one has $\xi \geq \frac{2}{2 m_{0}+1}$. Take $m=3 m_{0}+2,5 m_{0}+4, \cdots,(2 t+1) m_{0}+2 t$ successively. One gets from Inequality (2.3) that $\xi \geq \frac{4}{3 m_{0}+2}, \frac{7}{5 m_{0}+4}, \cdots, \frac{3 t+1}{(2 t+1) m_{0}+2 t}$. Taking the limit, we have $\xi \geq \frac{3}{2 m_{0}+2}$. By Inequality (2.1), we have

$$
K_{X}^{3} \geq \frac{3\left(P_{m_{0}}-2\right)}{2 m_{0}^{2}\left(m_{0}+1\right)} \geq \frac{3}{2 m_{0}^{2}\left(m_{0}+1\right)} .
$$

In fact, we have the following estimation for each small $m_{0}$, which slightly improves [7, Table A]:

Table A2

\begin{tabular}{|c|c|c|c|c|c|c|c|}
\hline$m_{0}=$ & 2 & 3 & 4 & 5 & 6 & 7 & 8 \\
\hline$\xi \geq$ & $1 / 2$ & $2 / 5$ & $1 / 3$ & $1 / 4$ & $2 / 9$ & $1 / 5$ & $1 / 6$ \\
\hline$K^{3} \geq$ & $1 / 8$ & $2 / 45$ & $1 / 48$ & $1 / 100$ & $1 / 162$ & $1 / 245$ & $1 / 384$ \\
\hline \hline$m_{0}=$ & 9 & 10 & 11 & 12 & 13 & 14 & 15 \\
\hline$\xi \geq$ & $2 / 13$ & $1 / 7$ & $1 / 8$ & $2 / 17$ & $1 / 9$ & $1 / 10$ & $2 / 21$ \\
\hline$K^{3} \geq$ & $2 / 1053$ & $1 / 700$ & $1 / 968$ & $1 / 1224$ & $1 / 1521$ & $1 / 1960$ & $2 / 4725$ \\
\hline
\end{tabular}

Under the same situation, if there exists a number $m_{1}>0$ such that $d_{m_{1}}=3$, then, since $\left(\left.m_{1} \pi^{*}\left(K_{X}\right)\right|_{F} \cdot C\right) \geq 2$, we have $\xi \geq \frac{2}{m_{1}}$. Thus Inequality (2.1) reads:

$$
K_{X}^{3} \geq \frac{2\left(P_{m_{0}}-2\right)}{m_{0}^{2} m_{1}} \geq \frac{2}{m_{0}^{2} m_{1}} .
$$

3.3. The case $d_{m_{0}}=1, b=g(\Gamma)>0$.

We have $S=F$ by definition. Pick a very large number $l>0$. Take $|G|:=\left|l \sigma^{*}\left(K_{F_{0}}\right)\right|$ which is base point free by the surface theory. By definition, we have $\theta \geq P_{m_{0}} \geq 2$. Since $\left.\pi^{*}\left(K_{X}\right)\right|_{F} \sim \sigma^{*}\left(K_{F_{0}}\right)$ by Lemma 2.1(i), we see $\beta=\frac{1}{l}$ and thus Inequality (2.1) implies

$$
K_{X}^{3} \geq \frac{P_{m_{0}}}{m_{0}} \cdot \frac{1}{l} \cdot l K_{F_{0}}^{2} \geq \frac{P_{m_{0}}}{m_{0}} .
$$

3.4. The case $d_{m_{0}}=1, b=0$.

By Lemma 2.1(ii), we have

$$
K_{X}^{3} \geq\left.\frac{\theta}{m_{0}} \pi^{*}\left(K_{X}\right)\right|_{F} ^{2} \geq \frac{\theta^{3}}{m_{0}\left(m_{0}+\theta\right)^{2}} \cdot K_{F_{0}}^{2} .
$$


We will choose suitable linear system $|G|$ on $F$ depending on the numerical type of $F$. From the surface theory, we know that either $K_{F_{0}}^{2} \geq 2$ or $\left(K_{F_{0}}^{2}, p_{g}(F)\right)=(1,2),(1,1),(1,0)$.

Subcase 3.4.1. $K_{F_{0}}^{2} \geq 2$.

Inequality (3.5) implies

$$
K_{X}^{3} \geq \frac{2 \theta^{3}}{m_{0}\left(m_{0}+\theta\right)^{2}} .
$$

Subase 3.4.2. $\left(K_{F_{0}}^{2}, p_{g}\left(F_{0}\right)\right)=(1,2)$.

Take $|G|:=\operatorname{Mov}\left|K_{F}\right|$. Then $C$, as a generic irreducible element of $|G|$, is a smooth curve of genus 2 (see [1]). By Lemma 2.1(ii), we have $\beta=\frac{\theta}{m_{0}+\theta} \geq \frac{1}{m_{0}+1}$.

Inequality (2.2) implies $\xi \geq \frac{\theta}{m_{0}+\theta}$. Take $m=\left\lfloor\frac{3 m_{0}+3 \theta}{\theta}\right\rfloor+1>\frac{3 m_{0}+3 \theta}{\theta}$. Then, since $\alpha_{m} \geq\left(m-1-\frac{m_{0}}{\theta}-\frac{1}{\beta}\right) \xi>1$, Inequality (2.3) gives $\xi \geq$ $\frac{4}{\left\lfloor\frac{3 m_{0}+3 \theta}{\theta}\right\rfloor+1} \geq \frac{4 \theta}{3 m_{0}+4 \theta}$. Inductively, take $m=\left\lfloor\frac{\left(1+\frac{2}{3}\left(4^{t}-1\right)\right) m_{0}+3 \cdot 4^{t-1} \theta}{4^{t-1} \theta}\right\rfloor+1$, one gets $\xi \geq \frac{4^{t} \theta}{\left(1+\frac{2}{3}\left(4^{t}-1\right)\right) m_{0}+4^{t} \theta}$ and hence $\xi \geq \frac{3 \theta}{2 m_{0}+3 \theta}$ by taking the limit. Thus we have

$$
K_{X}^{3} \geq \frac{3 \theta^{3}}{m_{0}\left(m_{0}+\theta\right)\left(2 m_{0}+3 \theta\right)} \geq \frac{3}{m_{0}\left(m_{0}+1\right)\left(2 m_{0}+3\right)} .
$$

A similar calculation leads to the following better estimation for smaller $m_{0}$ :

Table A3

\begin{tabular}{|c|c|c|c|c|c|c|c|}
\hline$m_{0}=$ & 2 & 3 & 4 & 5 & 6 & 7 & 8 \\
\hline$\xi \geq$ & $1 / 2$ & $1 / 3$ & $2 / 7$ & $1 / 4$ & $1 / 5$ & $2 / 11$ & $1 / 6$ \\
\hline$K^{3} \geq$ & $1 / 12$ & $1 / 36$ & $1 / 70$ & $1 / 120$ & $1 / 210$ & $1 / 308$ & $1 / 432$ \\
\hline \hline$m_{0}=$ & 9 & 10 & 11 & 12 & 13 & 14 & 15 \\
\hline$\xi \geq$ & $1 / 7$ & $2 / 15$ & $1 / 8$ & $1 / 9$ & $2 / 19$ & $1 / 10$ & $1 / 11$ \\
\hline$K^{3} \geq$ & $1 / 630$ & $1 / 825$ & $1 / 1056$ & $1 / 1404$ & $1 / 1729$ & $1 / 2100$ & $1 / 2640$ \\
\hline
\end{tabular}

Subcase 3.4.3. $\left(K_{F_{0}}^{2}, p_{g}\left(F_{0}\right)\right)=(1,1)$.

Since $\left|\sigma^{*}\left(K_{F_{0}}\right)\right|$ is not moving, we have to take $|G|:=\left|2 \sigma^{*}\left(K_{F_{0}}\right)\right|$ which is base point free by the surface theory. Naturally the generic irreducible element $C$ of $|G|$ is even and $\operatorname{deg}\left(K_{C}\right)=6$.

By Lemma 2.1(ii), we have $\beta=\frac{\theta}{2 m_{0}+2 \theta}$. Take $m=\left\lfloor\frac{3 m_{0}+3 \theta}{\theta}\right\rfloor+1$. Since $\xi>0$, we have $\alpha_{m}>0$. Thus Lemma 2.2 implies $\xi \geq \frac{8 \theta}{3 m_{0}+4 \theta}$. Thus Inequality (2.1) reads

$$
K_{X}^{3} \geq \frac{4 \theta^{3}}{m_{0}\left(m_{0}+\theta\right)\left(3 m_{0}+4 \theta\right)} .
$$

For each small $m_{0}$, we have the following better estimation:

Table A4 


\begin{tabular}{|c|c|c|c|c|c|c|c|}
\hline$m_{0}=$ & 2 & 3 & 4 & 5 & 6 & 7 & 8 \\
\hline$\xi \geq$ & $6 / 7$ & $2 / 3$ & $1 / 2$ & $4 / 9$ & $3 / 8$ & $1 / 3$ & $2 / 7$ \\
\hline$K^{3} \geq$ & $1 / 14$ & $1 / 36$ & $1 / 80$ & $1 / 135$ & $1 / 224$ & $1 / 336$ & $1 / 504$ \\
\hline \hline$m_{0}=$ & 9 & 10 & 11 & 12 & 13 & 14 & 15 \\
\hline$\xi \geq$ & $4 / 15$ & $6 / 25$ & $2 / 9$ & $1 / 5$ & $4 / 21$ & $14 / 79$ & $1 / 6$ \\
\hline$K^{3} \geq$ & $1 / 675$ & $3 / 2750$ & $1 / 1188$ & $1 / 1560$ & $1 / 1911$ & $1 / 2370$ & $1 / 2880$ \\
\hline
\end{tabular}

Subcase 3.4.4. $\left(K_{F_{0}}^{2}, p_{g}\left(F_{0}\right)\right)=(1,0)$.

Modulo further birational modification, we may assume that Mov $\left|2 K_{F}\right|$ is base point free. Take $|G|=\operatorname{Mov}\left|2 K_{F}\right|$. By Catanese-Pignatelli [4], the generic irreducible element $C$ of $|G|$ is a smooth curve of genus $\geq 3$. By Lemma 2.1(ii), we have $\beta=\frac{\theta}{2 m_{0}+2 \theta} \geq \frac{1}{2 m_{0}+2}$. Lemma 2.4 implies $\xi \geq \frac{\theta}{m_{0}+\theta} \cdot\left(\sigma^{*}\left(K_{F_{0}}\right) \cdot C\right) \geq \frac{2 \theta}{m_{0}+\theta}$. Thus we have

$$
K_{X}^{3} \geq \frac{\theta^{3}}{m_{0}\left(m_{0}+\theta\right)^{2}} .
$$

Of course, for each small $m_{0}$, one might get slightly better estimation for $\xi$ and $K_{X}^{3}$.

Variant 3.4.5. If there exists a positive integer $m_{1}$ such that $P_{m_{1}} \geq 2$ and that $\left|m_{0} K_{X^{\prime}}\right|$ and $\left|m_{1} K_{X^{\prime}}\right|$ are not composed with the same pencil. We may take $|G|=\left|M_{m_{1}}\right|_{F} \mid$ and then we have $\beta=\frac{1}{m_{1}}$. Thus Inequality (2.1) and Lemma 2.4 imply

$$
K_{X}^{3} \geq \frac{2 \theta_{m_{0}}^{2}}{m_{0} m_{1}\left(m_{0}+\theta_{m_{0}}\right)},
$$

provided that $\left(K_{F_{0}}^{2}, p_{g}\left(F_{0}\right)\right) \neq(1,2)$.

\subsection{Some other inequalities.}

Corollary 3.1. Let $X$ be a minimal 3-fold of general type. Assume $P_{m_{0}}=2$. Keep the same notation as above. Suppose that the general fiber $F$ of the induced fibration from $\Phi_{m_{0}}$ is not a $(1,2)$ surface, and that $P_{m_{1}} \geq 2$ for some integer $m_{1}>0$. Then

$$
K_{X}^{3} \geq \min \left\{\frac{\left(P_{m_{1}}-1\right)^{3}}{m_{1}\left(m_{1}+P_{m_{1}}-1\right)^{2}}, \frac{2}{m_{0} m_{1}\left(m_{0}+1\right)}\right\} .
$$

Proof. If $\left|m_{0} K_{X^{\prime}}\right|,\left|m_{1} K_{X^{\prime}}\right|$ are composed with the same pencil, then both $\left|m_{0} K_{X^{\prime}}\right|$ and $\left|m_{1} K_{X^{\prime}}\right|$ induce the same fibration $f: X^{\prime} \longrightarrow \Gamma$. Consider $\tilde{\Lambda}=\left|m_{1} K_{X^{\prime}}\right|$. Then, $\theta_{m_{1}} \geq P_{m_{1}}-1$. Since $F$ is not a $(1,2)$ surface and by comparing Inequality $3.4,3.6,3.8$ and 3.9, we have

$$
K_{X}^{3} \geq \frac{\left(P_{m_{1}}-1\right)^{3}}{m_{1}\left(m_{1}+P_{m_{1}}-1\right)^{2}} .
$$

Suppose that $\left|m_{0} K_{X^{\prime}}\right|,\left|m_{1} K_{X^{\prime}}\right|$ are not composed with the same pencil. We have $\beta=\frac{1}{m_{1}}$. Then we have Inequality (3.10) as in Variant 3.4.5. 
Now we are able to study the more restricted case:

Proposition 3.2. Let $X$ be a minimal 3-fold of general type. Assume that $P_{m_{0}}(X) \geq 4$ and $d_{m_{0}}=2$, then

$$
K_{X}^{3} \geq \min \left\{\frac{8}{m_{0}\left(m_{0}+2\right)^{2}}, \frac{6}{m_{0}^{2}\left(m_{0}+2\right)}\right\} .
$$

Proof. We need to study the image surface $W^{\prime}$ of $X^{\prime}$ through the morphism $\Phi_{\left|m_{0} K_{X^{\prime}}\right|}$. In fact, we have the Stein factorization

$$
\Phi_{m_{0}}:=\Phi_{\left|m_{0} K_{X^{\prime}}\right|}: X^{\prime} \stackrel{f}{\longrightarrow} \Gamma \stackrel{s}{\longrightarrow} W^{\prime} \subset \mathbb{P}^{P_{m_{0}}-1} .
$$

Denote by $H^{\prime}$ a very ample divisor on $W^{\prime}$ such that $M_{m_{0}} \sim \Phi_{m_{0}}^{*}\left(H^{\prime}\right)$. Furthermore one has $\left.M_{m_{0}}\right|_{S} \equiv \tilde{a}_{m_{0}} C$ for a general member $S \in\left|M_{m_{0}}\right|$ and the integer $\tilde{a}_{m_{0}} \geq \operatorname{deg}(s) \operatorname{deg}\left(W^{\prime}\right) \geq \operatorname{deg}\left(W^{\prime}\right) \geq P_{m_{0}}-2$, where $C$ is a general fiber of $f$. Set $|G|:=\left|M_{m_{0}}\right| S \mid$.

Case 1. $\tilde{a}_{m_{0}} \geq 3$.

We have $\beta \geq \frac{3}{m_{0}}$. Inequality (2.2) implies $\xi \geq \frac{6}{4 m_{0}+3}$. Take $m=$ $2 m_{0}+2$. Then Inequality (2.3) gives $\xi \geq \frac{2}{m_{0}+1}$. Take $m=\left\lfloor\frac{11 m_{0}+9}{6}\right\rfloor+1$. Since $\alpha_{m}>\left(\frac{11 m_{0}+9}{6}-1-m_{0}-\frac{1}{\beta}\right) \xi \geq 1$, Inequality (2.3) implies $\xi \geq \frac{24}{11 m_{0}+15}$. Thus, we have

$$
K_{X}^{3} \geq \frac{72}{m_{0}^{2}\left(11 m_{0}+15\right)} .
$$

Case 2. $\tilde{a}_{m_{0}}=2$.

Automatically we have $P_{m_{0}}=4$, which also implies that $\operatorname{deg}\left(W^{\prime}\right)=2$ and $\operatorname{deg}(s)=1$. Recall that an irreducible surface (in $\mathbb{P}^{3}$ ) of degree 2 is one of the following surfaces (see, for instance, Reid [28, p. 30, Ex. 19]):

(a) $W^{\prime}$ is the cone $\overline{\mathbb{F}}_{2}$ obtained by blowing down the unique section with the self-intersection $(-2)$ on the Hirzebruch ruled surface $\mathbb{F}_{2}$;

(b) $W^{\prime} \cong \mathbb{P}^{1} \times \mathbb{P}^{1}$.

Case 2.a. $W^{\prime}=\overline{\mathbb{F}}_{2}$.

Replacing by its birational model, we may assume that $\Phi_{m_{0}}$ factors through the minimal resolution $\mathbb{F}_{2}$ of $W^{\prime}$. So we have the factorization of $\Phi_{m_{0}}: X^{\prime} \stackrel{h}{\longrightarrow} \mathbb{F}_{2} \stackrel{\nu}{\longrightarrow} W^{\prime}$ where $h$ is a fibration and $\nu$ is the minimal resolution of $W^{\prime}$. Set $\hat{H}=\nu^{*}\left(H^{\prime}\right)$. We know that $H^{\prime 2}=2$ and hence $\hat{H}^{2}=2$. Noting that $\hat{H}$ is nef and big on $\mathbb{F}_{2}$, we can write

$$
\hat{H} \sim \mu G_{0}+n T
$$

where $\mu$ and $n$ are integers, $G_{0}$ denotes the unique section with $G_{0}^{2}=$ -2 , and $T$ is the general fiber of the ruling on $\mathbb{F}_{2}$. The property of $\hat{H}$ being nef and big implies that $\mu>0$ and $n \geq 2 \mu \geq 2$. Now let 
$p r: \mathbb{F}_{2} \longrightarrow \mathbb{P}^{1}$ be the ruling. Set $\tilde{f}:=p r \circ h: X^{\prime} \longrightarrow \mathbb{P}^{1}$, which is a fibration with connected fibers. Denote by $F$ a general fiber of $\tilde{f}$. We have

$$
M_{m_{0}} \sim \Phi_{m_{0}}^{*}\left(H^{\prime}\right)=h^{*}(\hat{H}) \geq 2 F .
$$

Let $\Lambda=|2 F| \preceq\left|m_{0} K_{X^{\prime}}\right|$. Clearly we have $\theta_{\Lambda}=2, d_{\Lambda}=1$ and $b=0$. By Inequalities (3.6), (3.7), (3.8) and (3.9), we have

$$
K_{X}^{3} \geq \frac{8}{m_{0}\left(m_{0}+2\right)^{2}} .
$$

Case 2.b. $W^{\prime}=\mathbb{P}^{1} \times \mathbb{P}^{1}$.

We have an induced fibration $f: X^{\prime} \longrightarrow W^{\prime}=\mathbb{P}^{1} \times \mathbb{P}^{1}$. Since a very ample divisor $H^{\prime}$ on $W^{\prime}$ with $H^{\prime 2}=2$ is linearly equivalent to $L_{1}+L_{2}=q_{1}^{*}$ (point) $+q_{2}^{*}$ (point) where $q_{1}, q_{2}$ are projections from $\mathbb{P}^{1} \times \mathbb{P}^{1}$ to $\mathbb{P}^{1}$ respectively. Set $\tilde{f}_{i}:=q_{i} \circ f: X^{\prime} \longrightarrow \mathbb{P}^{1}, i=1,2$. Then $\tilde{f}_{1}$ and $\tilde{f}_{2}$ are two fibrations onto $\mathbb{P}^{1}$. Let $F_{1}$ and $F_{2}$ be general fibers of $\tilde{f}_{1}$ and $\tilde{f}_{2}$, respectively. Then $F_{1} \cap F_{2}$ is simply a general fiber $C$ of $f$. We will estimate $\xi$ in an alternative way. In fact, the following argument is similar to the proof of [13, Theorem 3.1].

Since $\tilde{a}_{m_{0}}=2$, we have $\left.S\right|_{S} \sim 2 C$. On the other hand, we have $S \geq F_{1}+F_{2}$. Modulo further birational modifications, we may write $m_{0} \pi^{*}\left(K_{X}\right) \equiv F_{1}+F_{2}+H_{m_{0}}^{\prime}$ where $H_{m_{0}}^{\prime}$ is an effective $\mathbb{Q}$-divisor with simple normal crossing supports. For any integer $m>m_{0}+1$, we consider the linear system

$$
\left|K_{X^{\prime}}+\left\lceil\left(m-m_{0}-1\right) \pi^{*}\left(K_{X}\right)\right\rceil+F_{1}+F_{2}\right| \preceq\left|m K_{X^{\prime}}\right| .
$$

Since $\left(m-m_{0}-1\right) \pi^{*}\left(K_{X}\right)+F_{2}$ is nef and big, Kawamata-Viehweg vanishing $([19,33])$ gives the surjective map:

$$
\begin{aligned}
& H^{0}\left(K_{X^{\prime}}+\left\lceil\left(m-m_{0}-1\right) \pi^{*}\left(K_{X}\right)\right\rceil+F_{2}+F_{1}\right) \\
\longrightarrow & H^{0}\left(F_{1}, K_{F_{1}}+\left.\left\lceil\left(m-m_{0}-1\right) \pi^{*}\left(K_{X}\right)\right\rceil\right|_{F_{1}}+C\right) .
\end{aligned}
$$

Using the vanishing theorem again, one gets the surjective map:

$H^{0}\left(F_{1}, K_{F_{1}}+\left\lceil\left.\left(m-m_{0}-1\right) \pi^{*}\left(K_{X}\right)\right|_{F_{1}}\right\rceil+C\right) \longrightarrow H^{0}\left(C, K_{C}+\hat{D}_{m}\right)$

where $\hat{D}_{m}:=\left.\left\lceil\left.\left(m-m_{0}-1\right) \pi^{*}\left(K_{X}\right)\right|_{F_{1}}\right\rceil\right|_{C}$ with

$$
\operatorname{deg}\left(\hat{D}_{m}\right) \geq\left(m-m_{0}-1\right) \xi
$$

When $m$ is large enough so that $\operatorname{deg}\left(\hat{D}_{m}\right) \geq 2$, the above two surjective maps directly implies

$$
m \xi \geq \operatorname{deg}\left(K_{C}\right)+\operatorname{deg}\left(\hat{D}_{m}\right) \geq 2+\left\lceil\left(m-m_{0}-1\right) \xi\right\rceil .
$$

In particular, we have $\xi \geq \frac{2}{m_{0}+1}$.

Take $m=2 m_{0}+3$. Then $\left(m-m_{0}-1\right) \xi>2$ and Inequality (3.13) gives $\xi \geq \frac{5}{2 m_{0}+3}$.

Assume $m_{0}>1$ and take $m=2 m_{0}+2$. One gets $\xi \geq \frac{5}{2 m_{0}+2}$. Take $m=\left\lfloor\frac{7 m_{0}+12}{5}\right\rfloor=\left\lfloor\frac{7 m_{0}+7}{5}\right\rfloor+1>\frac{7 m_{0}+7}{5}$, one has $\xi \geq \frac{4}{m} \geq \frac{20}{7 m_{0}+12}$. 
Inductively, take $m=\left\lfloor\frac{\left(2+\frac{5}{3}\left(4^{t}-1\right)\right) m_{0}+2+\frac{10}{3}\left(4^{t}-1\right)}{5 \cdot 4^{t-1}}\right\rfloor$ for $t \geq 1$, one has $\xi \geq \frac{5 \cdot 4^{t}}{\left(2+\frac{5}{3}\left(4^{t}-1\right)\right) m_{0}+2+\frac{10}{3}\left(4^{t}-1\right)}$. We have $\xi \geq \frac{3}{m_{0}+2}$ by taking the limit and hence

$$
K_{X}^{3} \geq \frac{1}{m_{0}} \cdot\left(\left.\pi^{*}\left(K_{X}\right)\right|_{S}\right)^{2} \geq \frac{2}{m_{0}^{2}} \cdot \xi \geq \frac{6}{m_{0}^{2}\left(m_{0}+2\right)} .
$$

We conclude the statement by comparing 3.11 , 3.12 and 3.14 .

Corollary 3.3. Let $X$ be a minimal 3-fold of general type. The following holds:

$$
K_{X}^{3} \geq \begin{cases}\min \left\{\frac{8}{m_{0}\left(m_{0}+2\right)^{2}}, \frac{7}{2 m_{0}^{2}\left(m_{0}+1\right)}\right\}, & \text { when } P_{m_{0}} \geq 4 \\ \frac{3}{2 m_{0}^{2}\left(m_{0}+1\right)}, & \text { when } P_{m_{0}}=3\end{cases}
$$

Proof. When $P_{m_{0}} \geq 4, d_{m_{0}}=3,2,1$ and the inequality follows from comparing Inequality (3.1), Proposition 3.2, Inequalities (3.4, 3.6, 3.7, 3.8, 3.9) (with $\theta_{m_{0}}=3$ ), respectively.

When $P_{m_{0}}=3, d_{m_{0}}=2,1$ and the inequality follows immediately by comparing Inequality (3.2) with Inequalities (3.4, 3.6, 3.7, 3.8, 3.9) (with $\theta_{m_{0}}=2$ ).

\section{Threefolds with $\delta(V) \leq 12$}

The purpose of this section is to prove the following sharper bounds:

Theorem 4.1. Let $X$ be a minimal projective 3-fold of general type with $2 \leq \delta(X) \leq 12$. Then $K_{X}^{3} \geq v(\delta(X))$, where the function $v(x)$ is defined as follows:

\begin{tabular}{|c|c|c|c|c|c|c|}
\hline$x$ & 2 & 3 & 4 & 5 & 6 & 7 \\
\hline$v(x)$ & $1 / 14$ & $1 / 36$ & $1 / 90$ & $1 / 135$ & $1 / 224$ & $1 / 336$ \\
\hline \hline$x$ & 8 & 9 & 10 & 11 & 12 & -- \\
\hline$v(x)$ & $1 / 504$ & $1 / 675$ & $3 / 2750$ & $1 / 1188$ & $1 / 1560$ & -- \\
\hline
\end{tabular}

We are going to estimate the lower bound of the volume, case by case, for a given $\delta$. The discussion here relies on those formulae in [6, (3.6)-(3.12)]

Proposition 4.2. If $P_{2}(X) \geq 2$, then $K_{X}^{3} \geq \frac{1}{14}$.

Proof. Set $m_{0}=2$. By Table A1, Table A2, Inequalities (3.4) and (3.6), Table A3, Table A4 and Corollary 3.3, we have $K_{X}^{3} \geq \frac{1}{14}$ unless $P_{2}=2, d_{2}=1, b=0$ and $F$ is of type $(1,0)$.

In the remaining case, we have that $\chi\left(\mathcal{O}_{X}\right)=1$ by [7, Lemma 2.32]. By [7, Lemma 3.2], one has $P_{4} \geq 2 P_{2} \geq 4$. If $d_{4} \geq 2$, then $K_{X}^{3} \geq \frac{1}{12}$ by Inequality (3.10) (with $m_{0}=2, m_{1}=4, \theta_{2}=1$ ). If $d_{4}=1$, then $\left|2 K_{X^{\prime}}\right|$ and $\left|4 K_{X^{\prime}}\right|$ are composed with the same pencil. Thus we have $K_{X}^{3} \geq \frac{27}{196}>\frac{1}{8}$ by Inequality (3.9) (with $m_{0}=4, \theta_{4}=3$ ). 
Proposition 4.3. If $P_{3}(X) \geq 2$, then $K_{X}^{3} \geq \frac{1}{36}$.

Proof. Take $m_{0}=3$ and $\Lambda=\left|3 K_{X^{\prime}}\right|$. One has $K_{X}^{3} \geq \frac{1}{36}$ by Table A1, Table A2, Inequalities (3.4), (3.6), Table A3, Table A4 and Corollary $3.3\left(m_{0}=3\right)$ unless we are in Subcase 3.4.4 with $P_{3}=2$. That is, $P_{3}=2, d_{3}=1, b=0$ and $F$ is of type $(1,0)$. Again, $\chi\left(\mathcal{O}_{X}\right)=1$. Thus, for any $m \geq 2$, 7, Lemma 3.2] implies $P_{m+2} \geq P_{m}+P_{2}$.

By Corollary [3.1, if $P_{4} \geq 3$ (resp. $P_{5} \geq 3$ ), then $K_{X}^{3} \geq \frac{1}{24}$ (resp. $\frac{1}{30}$ ). Suppose that both $P_{4} \leq 2$ and $P_{5} \leq 2$, then $P_{5}=2$ and $P_{2}=0$. By [6, (3.6)], $n_{1,2}^{0}=5-8+P_{4}<0$, which is a contradiction. Hence either $P_{4}$ or $P_{5} \geq 3$ in this case and we are done.

Proposition 4.4. If $P_{4}(X) \geq 2$, then $K_{X}^{3} \geq \frac{1}{90}$.

Proof. Similarly, we have $K_{X}^{3} \geq \frac{1}{80}$ unless $P_{4}=2, b=0$ and $F$ is of $(1,0)$ type. In fact, in this situation, we have at least $K_{X}^{3} \geq \frac{1}{100}$ by Inequality (3.9). We will go a little bit further to investigate this situation.

0. We may and do assume that $P_{2} \leq 1$ and $P_{3} \leq 1$.

1. If $P_{7} \geq 3$ (resp. $P_{6} \geq 3, P_{5} \geq 3$ ), then $K^{3} \geq \frac{8}{567}>\frac{1}{80}$ (resp. $\frac{1}{60}, \frac{1}{50}$ ) by Corollary 3.1 (with $m_{0}=4$, and $m_{1}=7,6,5$ respectively). So we may assume $P_{5}, P_{6}, P_{7} \leq 2$. Since $P_{6} \geq P_{4}+P_{2}$, we see that $P_{2}=0$ and $P_{6}=P_{4}=2$.

2. If $P_{3}=0$, then $n_{1,3}^{0}=P_{5}-2 \geq 0$ implies $P_{5}=2$. Now $n_{1,4}^{5}=3-\sigma_{5} \geq$ 0 gives $\sigma_{5} \leq 3$. However $n_{1,3}^{5} \geq 0$ implies $\sigma_{5} \geq 4$, a contradiction. We thus assume that $P_{3}=1$ from now on.

3. We thus can make the following complete table for $B^{(5)}$ depending on $P_{5}, \sigma_{5}$ :

\begin{tabular}{|c|c|c||c|c|c|}
\hline No. & $P_{5}$ & $\sigma_{5}$ & $B^{(5)}$ & $K^{3}$ & $\epsilon+P_{7}$ \\
\hline 1 & 1 & 0 & $\{2 \times(1,2),(2,5), 5 \times(1,4)\}$ & $1 / 20$ & 4 \\
\hline 2 & 1 & 1 & $\{3 \times(1,2),(1,3), 4 \times(1,4),(1, r)\}$ & $1 / r-1 / 6$ & 4 \\
\hline 3 & 2 & 1 & $\{(1,2), 2 \times(2,5), 3 \times(1,4),(1, r)\}$ & $1 / r-3 / 20$ & 5 \\
\hline 4 & 2 & 2 & $\left\{2 \times(1,2),(2,5),(1,3), 2 \times(1,4),\left(1, r_{1}\right),\left(1, r_{2}\right)\right\}$ & $1 / r_{1}+1 / r_{2}-11 / 30$ & 5 \\
\hline 5 & 2 & 3 & $\left\{3 \times(1,2), 2 \times(1,3),(1,4),\left(1, r_{1}\right),\left(1, r_{2}\right),\left(1, r_{3}\right)\right\}$ & $1 / r_{1}+r_{2}+r_{3}-7 / 12$ & 5 \\
\hline
\end{tabular}

4. By definition, one has $\sigma_{5} \leq \epsilon \leq 2 \sigma_{5}$. Note that No. 1 is impossible because $\epsilon=0$ but $P_{7} \leq 2$ implies that $\epsilon \geq 2$, a contradiction. In No. $3, P_{5}=2$ implies $P_{7}=2$ and hence $\epsilon=3>2 \sigma_{5}$, a contradiction.

In No. 2 , one must have $P_{7}=2$ and $\epsilon=2=2 \sigma_{5}$. Hence $r \geq 6$. Then it follows that $K^{3} \leq K^{3}\left(B^{(5)}\right) \leq 0$, a contradiction. Similarly, in No. $4, K^{3}\left(B^{(5)}\right)>0$ only when $r_{1}=r_{2}=5$. But then $\epsilon=2$, a contradiction.

5. It remains to consider No. 5. Note that $K^{3}\left(B^{(5)}\right)>0$ only when $r_{1}=r_{2}=r_{3}=5$ and $K^{3}\left(B^{(5)}\right)=\frac{1}{60}$. There are only finitely many possible packings. Among them, we search for baskets with $K^{3} \geq \frac{1}{100}$. It turns out there is only one new baskets

$$
B_{90}=\{3 \times(1,2), 2 \times(1,3),(2,9), 2 \times(1,5)\}
$$


with $K^{3}\left(B_{90}\right)=\frac{1}{90}$.

Proposition 4.5. If $P_{5} \geq 2$, then $K_{X}^{3} \geq \frac{1}{135}$.

Proof. Similarly, we have $K_{X}^{3} \geq \frac{1}{135}$ unless $P_{5}=2, b=0$ and $F$ a $(1,0)$ surface, for which we have $K_{X}^{3} \geq \frac{1}{180}$. Furthermore, we may assume that $P_{m} \leq 2$ for $m=6,7,8$ by Corollary [3.1. It suffices to consider: $\chi\left(\mathcal{O}_{X}\right)=1, P_{2}=0, P_{3}=0,1, P_{4}=0,1, P_{5}=P_{7}=2$ and $P_{4} \leq P_{6} \leq P_{8} \leq 2$.

We look at $B^{(5)}$ with $K^{3}>0$ according to $\left(P_{3}, P_{4}, P_{6}\right)$ and $\sigma_{5}$. It turns out that there is only one,

$$
B^{(5)}=\{2 \times(2,5), 3 \times(1,3),(1,4),(1,6)\}
$$

with $K^{3}\left(B^{(5)}\right)=\frac{1}{60}$, given by $\left(P_{3}, P_{4}, P_{6}\right)=(1,1,2)$ and $\sigma_{5}=2$. Now $P_{8}=2$ and hence

$$
B^{(7)}=\{2 \times(2,5), 2 \times(1,3),(2,7),(1,6)\} .
$$

However $K^{3}\left(B^{(7)}\right)=\frac{1}{210}<\frac{1}{180}$, which is impossible.

Proposition 4.6. If $P_{6} \geq 2$, then $K_{X}^{3} \geq \frac{1}{224}$.

Proof. Similarly, we have $K_{X}^{3} \geq \frac{1}{224}$ unless $P_{6}=2, b=0$ and $F$ a $(1,0)$ surface, for which we have $K_{X}^{3} \geq \frac{1}{294}$. Again, we may assume that $P_{m} \leq 2$ for $m=7,8,9,10$. Therefore, it remains to consider such a situation that $\chi\left(\mathcal{O}_{X}\right)=1, P_{2}=0, P_{4} \leq 1, P_{3} \leq P_{5} \leq 1, P_{7} \leq P_{9} \leq 2$ and $P_{8}=P_{10}=2$. According to the value of $\left(P_{3}, P_{4}, P_{5}\right)$ and $\sigma_{5}$, we have the following table.

\begin{tabular}{|c|c|c||c|c|c|}
\hline No. & $\left(P_{3}, P_{4}, P_{5}\right)$ & $\sigma_{5}$ & $B^{(5)}$ & $K^{3}$ & $\epsilon+P_{7}$ \\
\hline 1 & $(0,0,0)$ & 0 & $\{5 \times(1,2), 4 \times(1,3),(1,4)\}$ & $1 / 12$ & 2 \\
\hline 2 & $(0,0,1)$ & 0 & $\{3 \times(1,2), 2 *(2,5), 3 *(1,3)\}$ & $1 / 10$ & 3 \\
\hline 3 & $(0,1,0)$ & 0 & $\{6 *(1,2),(1,3), 3 *(1,4)\}$ & $1 / 12$ & 3 \\
\hline 4 & $(0,1,1)$ & 0 & $\{4 *(1,2), 2 *(2,5), 2 *(1,4)\}$ & $1 / 10$ & 4 \\
\hline 5 & $(0,1,1)$ & 1 & $\{5 *(1,2), 1 *(2,5),(1,3),(1,4),(1, r)\}$ & $1 / r-7 / 60$ & 4 \\
\hline 6 & $(0,1,1)$ & 2 & $\left\{6 *(1,2), 2 *(1,3),\left(1, r_{1}\right),\left(1, r_{2}\right)\right\}$ & $1 / r_{1}+1 / r_{2}-1 / 3$ & 4 \\
\hline 7 & $(1,0,1)$ & 0 & $\{(2,5), 6 *(1,3),(1,4)\}$ & $1 / 20$ & 2 \\
\hline 8 & $(1,0,1)$ & 1 & $\{(1,2), 7 *(1,3),(1, r)\}$ & $1 / r-1 / 6$ & 2 \\
\hline 9 & $(1,1,1)$ & 0 & $\{(1,2),(2,5), 3 *(1,3), 3 *(1,4)\}$ & $1 / 20$ & 3 \\
\hline 10 & $(1,1,1)$ & 1 & $\{2 *(1,2), 4 *(1,3), 2 *(1,4),(1, r)\}$ & $1 / r-1 / 6$ & 3 \\
\hline
\end{tabular}

1. It is clear that No. 2, 3, 4, 9 are not allowed for $\epsilon=0$ and hence $P_{7} \geq 3$.

2. In No. 1,7 , the baskets allow at most one packing at level 7 , i,e, $\epsilon_{7} \leq 1$. However, $P_{7}=2$ and $P_{8}=2$ yield $\epsilon_{7} \geq 2$, a contradiction.

3. Consider No. 10. Since $K^{3}=\frac{1}{r}-\frac{1}{6}>0$, it follows that $r=5$. So $\epsilon=1$ and $P_{7}=2$. Then $\epsilon_{7}=2$ and

$$
B^{(7)}=\{2 \times(1,2), 2 \times(1,3), 2 \times(2,7),(1,5)\} .
$$

This already implies $\epsilon_{8}=0$ and so we get $P_{9}=3$, a contradiction.

4. Consider No. 8. Since $K^{3}>0$, thus we get

$$
B^{(5)}=\{(1,2), 7 \times(1,3),(1,5)\} .
$$


Since $B^{(5)}$ allows no further packing, hence $K_{X}^{3}=\frac{1}{30}$ in this case.

5. Consider No. 5. Since $K^{3}>0, r=6,7,8$. It is easy to see that the basket with the smallest volume and dominated by $B^{(5)}$ is

$$
B_{210}=\{(7,15),(2,7),(1,6)\}
$$

with $K^{3}=\frac{1}{210}$. Thus $K_{X}^{3} \geq \frac{1}{210}$.

6. Finally Consider No. 6. Since $K^{3}>0,\left(r_{1}, r_{2}\right)=(5,5),(5,6),(5,7)$. It is easy to see that the basket with the smallest volume and dominated by $B^{(5)}$ is

$$
B_{105}=\{6 \times(1,2), 2 \times(1,3),(1,5),(1,7)\}
$$

with $K^{3}=\frac{1}{105}$. Thus $K_{X}^{3} \geq \frac{1}{105}$.

Note that, when $\delta(X) \geq 7$, we can utilize our explicit classification in [7, Section 3]. We shall omit some details to avoid unnecessary redundancy.

Proposition 4.7. If $P_{7} \geq 2$, then $K_{X}^{3} \geq \frac{1}{336}$.

Proof. Similarly, we have $K_{X}^{3} \geq \frac{1}{336}$ unless $P_{7}=2, b=0, F$ a $(1,0)$ surface and $\chi\left(\mathcal{O}_{X}\right)=1$. Again, we may assume that $P_{m} \leq 2$ for $m=8,9$. Hence $P_{9}=2$ and $P_{2}=0$.

By $\epsilon_{6}=0$, we have $P_{4}+P_{5}+P_{6}=P_{3}+2+\epsilon$. Hence $\left(P_{3}, P_{4}, P_{5}, P_{6}\right)=$ $(0,0,1,1),(0,1,0,1),(0,1,1,1)$ or $(1,1,1,1)$ which corresponds to Cases IV, V, VI, and VIII in [7, Section 3] respectively. The classification implies that, if $K_{X}^{3}<\frac{1}{336}$, then $B_{X} \succeq B_{\min }$, where $B_{\min }$ is a minimal positive basket and belongs to one of the following:

(b1) $B_{6,4}=\{(1,2),(6,13),(1,3), 2 \times(1,5)\}$ with $K^{3}\left(B_{6,4}\right)=\frac{1}{390}$ and $P_{9}\left(B_{6,4}\right)=3$

(b2) $B_{6,6}=\{3 \times(1,2),(3,7),(2,5),(1,4),(1,6)\}$ with $K^{3}\left(B_{6,6}\right)=\frac{1}{420}$ and $P_{9}\left(B_{6,4}\right)=3$;

(b3) $B_{8,3}=\{2 \times(2,5),(1,3),(3,11),(1,4)\}$ with $K^{3}\left(B_{8,3}\right)=\frac{1}{660}$.

Clearly, Case b1 can not happen because $P_{9}\left(B_{X}\right) \geq P_{9}\left(B_{\min }\right)=3$.

In the Case b2, for the similar reason, $B_{X} \neq B_{6,6}$. Thus $B_{X} \succeq B_{60}:=$ $\{4 \times(1,2), 2 \times(2,5),(1,4),(1,6)\}$ and so $K_{X}^{3} \geq K^{3}\left(B_{60}\right)=\frac{1}{60}$.

Finally, in Case b3, the proof of [7, Theorem 3.11] implies that $B_{X} \neq$ $B_{8,3}$ and $B_{X} \succeq B_{210}=\{2 \times(2,5),(1,3),(2,7), 2 \times(1,4)\}$ with $K_{X}^{3} \geq$ $K^{3}\left(B_{210}\right)=\frac{1}{210}$. We have proved the statement.

It is now immediately to see the following consequences:

Corollary 4.8. (=Corollary 1.5) Let $X$ be a minimal projective 3-fold of general type with $K_{X}^{3}<\frac{1}{336}$. Then $\delta(X) \geq 8$.

Proposition 4.9. Let $X$ be a minimal projective 3-fold of general type.

(1) If $P_{8} \geq 2$, then $K_{X}^{3} \geq \frac{1}{504}$.

(2) If $P_{9} \geq 2$, then $K_{X}^{3} \geq \frac{1}{675}$.

(3) If $P_{10} \geq 2$, then $K_{X}^{3} \geq \frac{3}{2750}$. 
(4) If $P_{11} \geq 2$, then $K_{X}^{3} \geq \frac{1}{1188}$.

(5) If $P_{12} \geq 2$, then $K_{X}^{3} \geq \frac{1}{1560}$

Proof. We only prove (1). Other statements can be proved similarly.

When $P_{8} \geq 2$, Table A1, Table A2, Inequalities (3.4). (3.6), Table A3 and Table A4 imply $K_{X}^{3} \geq \frac{1}{504}$ unless we are in Subcase 3.4.4, for which one has $K_{X}^{3} \geq \frac{1}{420}$ by [7, Theorem 1.2(2)] since $\chi\left(\mathcal{O}_{X}\right)=1$.

Propositions 4.2, 4.3, 4.4, 4.5, 4.6, 4.7 and 4.9 imply Theorem 4.1.

An interesting by-product is the following:

Corollary 4.10. (=Corollary 1.7(1)) Let $X$ be a minimal projective 3-fold of general type with $p_{g}(X)=1$. Then $K_{X}^{3} \geq \frac{1}{75}$.

Proof. We distinguish the following cases.

Case 1. $P_{4} \geq 3$.

By Corollary [3.3, $K_{X}^{3} \geq \frac{3}{160}$.

Case 2. $P_{4}=2$.

We have $K_{X}^{3} \geq \frac{1}{70}$ by Inequalities (3.4), (3.6) and Table A3 unless $b=0$ and $F$ is either a $(1,1)$ or a $(1,0)$ surface, for which we necessarily have $h^{2}\left(\mathcal{O}_{X}\right)=0$ and thus $\chi\left(\mathcal{O}_{X}\right)=0$. Reid's Riemann-Roch formula implies $P_{5}>P_{4}=2$. Now Corollary 3.1)(with $m_{0}=4, m_{1}=5$ ) yields $K_{X}^{3} \geq \frac{1}{50}$.

Case 3. $P_{4}=1$.

Since $p_{g}(X)=1$, one has $P_{m}>0$ for all $m>1$. By [6, (3.10)], we have

$$
P_{4}+P_{5}+P_{6}=3 P_{2}+P_{3}+P_{7}+\epsilon \geq 3 P_{2}+P_{3}+P_{7} .
$$

If $P_{4}=1$ (which implies $P_{3}=P_{2}=1$ ), then we have

$$
P_{5} \geq\left(P_{7}-P_{6}\right)+3 \geq 3 .
$$

Then, from [6, (3.6)], $n_{1,4}^{0} \geq 0$ implies $\chi\left(\mathcal{O}_{X}\right) \geq 3$. Due to our previous result [5, Corollary 1.2] for irregular 3-folds, we may assume $q(X)=0$. Thus we have $h^{2}\left(\mathcal{O}_{X}\right)=\chi\left(\mathcal{O}_{X}\right) \geq 3$. Take a sub-pencil $\Lambda$ of $\left|5 K_{X}\right|$. Then $\Lambda$ induces a fibration $f: X^{\prime} \longrightarrow \Gamma$ after Stein factorization. Let $F$ be the general fiber and $F_{0}$ be the minimal model of $F$.

Claim. $K_{F_{0}}^{2} \geq 2$.

Proof. Clearly we may write

$$
f_{*} \omega_{X^{\prime}}=\mathcal{O}_{\Gamma} \oplus \mathcal{O}_{\Gamma}\left(e_{2}\right) \oplus \cdots \oplus \mathcal{O}_{\Gamma}\left(e_{p_{g}(F)-1}\right)
$$

with $-2 \leq e_{j} \leq-1$ for all $j$, since $p_{g}\left(X^{\prime}\right)=1$. Note that we have

$$
\begin{aligned}
h^{2}\left(\mathcal{O}_{X}\right) & =h^{1}\left(f_{*} \omega_{X^{\prime}}\right)+h^{0}\left(R^{1} f_{*} \omega_{X^{\prime}}\right) \\
& \leq\left(p_{g}(F)-1\right)+h^{0}\left(R^{1} f_{*} \omega_{X^{\prime}}\right) .
\end{aligned}
$$

If $q(F)>0$, we have $K_{F_{0}}^{2} \geq 2$ by the surface theory. If $q(F)=0$, we have $R^{1} f_{*} \omega_{X^{\prime}}=0$ and thus $p_{g}(F) \geq h^{2}\left(\mathcal{O}_{X}\right)+1 \geq 4$. Hence we have $K_{F_{0}}^{2} \geq 4$ by the Noether inequality. 
If $d_{5} \geq 2$, then we may set $m_{1}=5$ and apply Inequality (3.10), which gives $K_{X}^{3} \geq \frac{1}{75}$.

If $d_{5}=1$, then $\left|5 K_{X^{\prime}}\right|$ and $\Lambda$ are composed with the same pencil. Thus we have $\theta_{5} \geq 2$ and Inequality (3.6) gives $K_{X}^{3} \geq \frac{16}{245}$.

\section{Threefolds with $\delta(V) \geq 13$}

Let $X$ be a minimal projective 3 -fold of general type with $\delta(X) \geq$ 13 . Now we are in the natural position to classify baskets $\mathbb{B}(X)$ with $\delta(X) \geq 13$. In fact, we have $\mathbb{B}^{12} \succeq \mathbb{B}(X) \succeq \mathbb{B}_{\text {min }}$ for certain minimal positive basket $\mathbb{B}_{\text {min }}$ listed in $[7$, Table $C]$, where $\mathbb{B}^{12}$ is also listed there. However, as pointed out in [7, Proposition 4.5], our earlier classification in [7, Table $\mathrm{C}$ ] is not clean since some minimal baskets in Table $\mathrm{C}$ are actually known to be "non-geometric".

Recall that, by definition, a geometric weighted basket is a basket of a projective threefold of general type. Hence the following properties hold:

A. $P_{m} P_{n} \leq P_{m+n}$ if $P_{m}=1$ and $n>0$.

B. $P_{m} \geq 0$ for all $m>0$.

C. $K^{3} \geq f\left(m_{0}\right)$ for some explicit function $f(x)$ given in Sections 3 and 4 provided that $P_{m_{0}} \geq 2$.

Indeed, if $\mathbb{B}^{12}$ violates one of $A, B, C$, then so does $\mathbb{B}(X)$. Therefore $\mathbb{B}(X)$ is non-geometric. If $\mathbb{B}_{\min }$ is non-geometric (e.g. cases No. $3 \mathrm{a}, 5 \mathrm{~b}$, $10 \mathrm{a}, \cdots$, etc.), then we need to check all baskets between $\mathbb{B}^{12}$ and $\mathbb{B}_{\min }$. The following Table $\mathrm{H}$ consists of non-geometric baskets with $\delta \geq 13$. We keep the same notation as in Table C.

\section{Table H}

\begin{tabular}{|l|c|c|c|l|}
\hline No. & $\left(P_{12}, \cdots, P_{24}\right)$ & $\left(n_{1,2}, n_{4,9}, \cdots, n_{1,5}\right)$ or $B_{\min }$ & $K^{3}$ & Offending \\
\hline $3 a$ & $(1,0,0,1,0,0,2,0,3,1,1,1,3)$ & $\{(2,5),(3,8), *\} \succ\{(5,13), *\}$ & $\frac{17}{30030}$ & $P_{8} P_{8}>P_{16}$ \\
$5 b$ & $(1,0,1,2,0,0,3,0,2,1,2,2,3)$ & $\{(5,13),(4,15), *\}$ & $\frac{1}{1170}$ & $P_{8} P_{8}>P_{16}$ \\
8 & $(1,0,2,1,0,1,3,1,4,3,2,2,5)$ & $(7,1,0,1,0,2,0,0,6,0,2,0,0,0,1)$ & $\frac{7^{7}}{77^{0}}$ & $P_{6} P_{10}>P_{16}$ \\
9 & $(1,0,2,-1,1,0,2,0,1,2,1,0,2)$ & $(9,0,0,2,0,0,1,1,4,0,1,0,0,1,0)$ & $\frac{1}{5544}$ & $P_{15}=-1$
\end{tabular}




\begin{tabular}{|c|c|c|c|c|}
\hline $10 a$ & $(1,0,2,1,2,-1,2,0,2,2,1,2,4)$ & $\{(4,9),(3,7), *\} \succ\{(7,16), *\}$ & $\frac{1}{1680}$ & $P_{17}=-1$ \\
\hline $11 a$ & $(1,0,2,0,2,0,2,2,2,1,1,1,3)$ & $\{(3,8),(4,11), *\} \succ\{(7,19), *\}$ & $\frac{1}{2660}$ & $P_{8} P_{14}>P_{22}$ \\
\hline 13 & $(1,0,3,-1,1,1,3,1,3,3,3,1,4)$ & $(12,0,0,2,0,2,0,2,4,0,2,0,0,1,0)$ & $\frac{4}{3465}$ & $P_{15}=-1$ \\
\hline $15 a$ & $(1,0,3,0,1,0,2,0,3,1,1,1,4)$ & $\{(4,11),(1,3), *\} \succ\{(5,14), *\}$ & $\frac{1}{2520}$ & $P_{8} P_{14}>P_{22}$ \\
\hline $15 b$ & $(1,0,2,0,1,0,3,0,3,2,1,1,4)$ & $\{(2,5),(3,8), *\} \succ\{(5,13), *\}$ & $\frac{23}{36,036}$ & $P_{8} P_{14}>P_{22}$ \\
\hline $15 c$ & $(1,0,3,1,2,0,3,1,3,2,2,2,5)$ & $\{(7,16),(7,19), *\}$ & $\frac{31}{31920}$ & $P_{8} P_{14}>P_{22}$ \\
\hline $16 c$ & $(1,0,2,1,1,-1,3,-1,2,2,1,1,3)$ & $\{\{(5,13),(7,16) *\}$ & $\frac{3}{16016}$ & $P_{17}=-1$ \\
\hline $18 a$ & $(1,0,3,0,1,0,2,1,2,2,2,1,3)$ & $\{(4,11),(1,3), *\} \succ\{(5,14), *\}$ & & $P_{6} P_{11}>P_{17}$ \\
\hline 19 & $(1,0,2,0,1,1,3,0,2,2,2,1,3)$ & $(8,0,1,1,0,1,0,1,5,0,1,0,0,1,0)$ & & $P_{9} P_{14}>P_{23}$ \\
\hline $20 a$ & $(1,0,1,1,1,0,3,-1,2,1,0,1,3)$ & $\{(2,5),(3,8), *\} \succ\{(5,13), *\}$ & & $P_{19}=-1$ \\
\hline $21 a$ & $(1,1,1,1,2,0,2,1,2,1,2,2,3)$ & $\{(1,3),(3,10), *\} \succ\{(4,13), *\}$ & & $P_{8} P_{9}>P_{17}$ \\
\hline 22 & $(1,0,1,1,1,0,2,1,3,1,1,1,3)$ & $(7,1,0,1,0,1,1,0,5,1,0,0,1,0,1)$ & $\frac{1}{9240}$ & $P_{8} P_{9}>P_{17}$ \\
\hline $23 a$ & $(1,0,2,1,2,0,2,1,3,1,2,2,3)$ & $\{(4,9),(3,7), *\} \succ\{(7,16), *\}$ & $\frac{1}{2640}$ & $P_{8} P_{9}>P_{17}$ \\
\hline 24 & $(1,0,2,0,0,1,3,0,3,2,2,0,3)$ & $(10,1,0,1,0,3,0,1,6,0,2,0,0,1,0)$ & $\frac{1}{3465}$ & $P_{8} P_{8}>P_{16}$ \\
\hline $26 a$ & $(1,0,3,1,1,1,3,0,4,1,2,2,5)$ & $\{(4,11),(1,3), *\} \succ\{(5,14), *\}$ & & $P_{9} P_{10}>P_{19}$ \\
\hline 27.1 & $(1,0,2,2,1,1,5,0,4,3,3,3,6)$ & $\{(2,5),(3,8), *\} \succ\{(5,13), *\}$ & & $P_{9} P_{10}>P_{19}$ \\
\hline 27.2 & $(1,0,2,2,1,1,5,-1,3,2,2,2,4)$ & $\{(2,5),(5,13), *\} \succ\{(7,18), *\}$ & $\frac{1}{1386}$ & $P_{19}=-1$ \\
\hline $27 a$ & $(1,0,2,2,1,1,5,-1,3,2,2,2,3)$ & $\{(2,5),(7,18), *\} \succ\{(9,23), *\}$ & $\frac{1}{1386}$ & $P_{19}=-1$ \\
\hline $27 b$ & $(1,0,2,2,1,1,5,-1,3,2,2,2,5)$ & $\{(5,13),(5,18), *\}$ & & $P_{19}=-1$ \\
\hline $29 a$ & $(1,1,3,1,2,2,2,1,3,1,2,2,3)$ & $\{(5,14),(1,3), *\} \succ\{(6,17), *\}$ & $\frac{1}{5335}$ & $P_{9} P_{14}>P_{23}$ \\
\hline $32 b$ & $(1,0,3,1,1,1,3,1,3,2,3,2,4)$ & $\{(4,11),(1,3), *\} \succ\{(5,14), *\}$ & $\frac{1}{1386}$ & $P_{9} P_{14}>P_{23}$ \\
\hline $33 a$ & $(1,1,2,0,2,1,1,1,2,2,1,2,3)$ & $\{(3,10),(2,7), *\} \succ\{(5,17), *\}$ & $\frac{1}{2856}$ & $P_{6} P_{16}>P_{22}$ \\
\hline $34 b$ & $(1,1,2,0,1,1,3,0,3,3,1,2,4)$ & $\{(2,5),(3,8), *\} \succ\{(5,13), *\}$ & $\frac{1}{1170}$ & $P_{6} P_{13}>P_{19}$ \\
\hline $39 a$ & $(1,1,2,1,3,0,2,1,3,2,2,3,4)$ & $\{(4,9),(3,7), *\} \succ\{(7,16), *\}$ & $\frac{1}{1680}$ & $P_{6} P_{16}>P_{22}$ \\
\hline $39 b$ & $(1,1,2,1,3,1,2,1,3,2,2,3,5)$ & $\{(3,10),(2,7), *\} \succ\{(5,17), *\}$ & & $P_{6} P_{16}>P_{22}$ \\
\hline 40.1 & $(1,1,2,1,2,1,4,0,4,3,2,3,6)$ & $\{(2,5),(3,8), *\} \succ\{(5,13), *\}$ & & $P_{6} P_{13}>P_{19}$ \\
\hline $40 a$ & $(1,1,2,1,2,1,4,-1,3,2,1,2,4)$ & $\{(4,10),(3,8), *\} \succ\{(7,18), *\}$ & $\frac{1}{2520}$ & $P_{6} P_{13}>P_{19}$ \\
\hline $40 b$ & $(1,1,2,1,2,1,4,0,4,3,1,2,5)$ & $\{(2,5),(6,16), *\} \succ\{(8,21), *\}$ & & $P_{6} P_{13}>P_{19}$ \\
\hline $43 a$ & $(1,1,3,0,2,1,2,1,3,2,2,2,4)$ & $\{(4,11),(1,3), *\} \succ\{(5,14), *\}$ & & $P_{7} P_{8}>P_{15}$ \\
\hline $43 b$ & $(1,1,2,0,2,1,3,1,3,3,2,2,4)$ & $\{(2,5),(3,8), *\} \succ\{(5,13), *\}$ & $\frac{23}{36036}$ & $P_{7} P_{8}>P_{15}$ \\
\hline $44 a$ & $(1,1,2,1,2,1,4,1,3,4,2,2,4)$ & $\{(2,5),(6,16), *\} \succ\{(8,21), *\}$ & & $P_{7} P_{18}>P_{25}=3$ \\
\hline $44 b$ & $(1,1,2,1,2,0,3,0,2,3,2,2,3)$ & $\{(7,16),(5,13), *\}$ & & $P_{7} P_{10}>P_{17}$ \\
\hline $46 a$ & $(1,1,1,1,2,1,3,0,3,1,1,2,3)$ & $\{(2,5),(3,8), *\} \succ\{(5,13), *\}$ & & $P_{9} P_{10}>P_{19}$ \\
\hline $50 a$ & $(1,1,3,1,2,2,3,1,4,2,3,3,5)$ & $\{(4,11),(1,3), *\} \succ\{(5,14), *\}$ & $\frac{1}{1260}$ & $P_{7} P_{14}>P_{21}$ \\
\hline $51 a$ & $(1,1,2,2,2,2,5,0,3,3,3,3,4)$ & $\{(4,10),(3,8), *\} \succ\{(7,18), *\}$ & & $P_{6} P_{13}>P_{19}$ \\
\hline $51 b$ & $(1,1,2,2,2,2,5,0,3,3,3,3,5)$ & $\{(5,13),(5,18), *\}$ & $\frac{1}{1170}$ & $P_{6} P_{13}>P_{19}$ \\
\hline $52 a$ & $(1,1,2,1,1,0,2,1,2,2,1,2,3)$ & $\{(2,5),(3,8), *\} \succ\{(5,13), *\}$ & $\frac{1}{2184}$ & $P_{5} P_{12}>P_{17}$ \\
\hline $56 a$ & $(1,1,2,2,1,1,2,1,3,2,2,3,3)$ & $\{(4,9),(3,7), *\} \succ\{(7,16), *\}$ & $\frac{1}{1680}$ & $P_{5} P_{14}>P_{19}$ \\
\hline 57 & $(1,0,2,2,0,1,3,1,3,2,2,2,3)$ & $(3,0,1,2,0,5,0,0,4,0,0,1,0,0,0)$ & $\frac{1}{1386}$ & $P_{7} P_{9}>P_{16}$ \\
\hline $58 a$ & $(1,1,2,2,2,0,2,1,3,2,2,3,4)$ & $\{(4,9),(3,7), *\} \succ\{(7,16), *\}$ & $\frac{1}{1680}$ & $P_{5} P_{12}>P_{17}$ \\
\hline $59 a$ & $(1,1,2,1,2,1,2,3,2,2,2,2,3)$ & $\{(3,8),(4,11), *\} \succ\{(7,19), *\}$ & $\frac{1}{2660}$ & Item C \\
\hline $60 a$ & $(1,1,1,2,1,1,3,0,3,1,1,2,3)$ & $\{(2,5),(3,8), *\} \succ\{(5,13), *\}$ & $\frac{1}{16380}$ & $P_{9} P_{10}>P_{19}$ \\
\hline 61 & $(1,1,1,2,1,1,2,2,3,2,2,2,3)$ & $(0,1,0,1,0,3,1,0,2,0,0,0,1,0,0)$ & $\frac{1}{9240}$ & Item $\mathrm{C}$ \\
\hline $62 a$ & $(1,1,2,2,2,1,2,2,3,2,3,3,3)$ & $\{(4,9),(3,7), *\} \succ\{(7,16), *\}$ & $\frac{1}{2640}$ & Item $\mathrm{C}$ \\
\hline 63 & $(1,1,3,1,2,1,3,2,3,3,2,2,4)$ & $(5,0,1,2,0,1,1,1,3,0,1,0,0,0,1)$ & $\frac{1}{5544}$ & Item $\mathrm{C}$ \\
\hline
\end{tabular}

By eliminating non-geometric baskets, we obtain a shorter list of baskets, listed in Table F-0, F-1, F-2 in the Appendix. We summarize some observations from the Tables.

Theorem 5.1. (=Theorem 1.4) Let $X$ be a minimal projective 3-fold of general type with the weighted basket $\mathbb{B}(X):=\left\{B_{X}, P_{2}, \chi\left(\mathcal{O}_{X}\right)\right\}$. If $\delta(X) \geq 13$, then $P_{2}=0$ and $\mathbb{B}(X)$ belongs to one of the types listed in Tables F-0 F-2 in Appendix. Furthermore, the following holds:

(1) $\delta(X)=18$ if and only if $\mathbb{B}(X)=\left\{B_{2 a}, 0,2\right\}$ (see Table F-0 for $\left.B_{2 a}\right)$ with $K_{X}^{3}=\frac{1}{1170}$.

(2) $\delta(X) \neq 16,17$. 
(3) $\delta(X)=15$ if and only if $\mathbb{B}(X)$ is among one of the cases in Table $F-1$. One has $K_{X}^{3} \geq \frac{1}{1386}$.

(4) $\delta(X)=14$ if and only if $\mathbb{B}(X)$ is among one of the cases in Table F-2. One has $K_{X}^{3} \geq \frac{1}{1680}$.

(5) $\delta(X)=13$ if and only if $\mathbb{B}(X)=\left\{B_{41}, 0,2\right\}$ (see Table $F-0$ for $\left.B_{41}\right)$ with $K_{X}^{3}=\frac{1}{252}$.

Theorem 4.1, Theorem 5.1 and [11, Theorem 1.4] imply the following:

Corollary 5.2. (=Theorem 1.6(2)) Let $X$ be a minimal projective 3fold of general type. Then $K_{X}^{3} \geq \frac{1}{1680}$, and equality holds if and only if $\chi\left(\mathcal{O}_{X}\right)=2, P_{2}=0$ and $B_{X}=B_{7 a}$ or $B_{X}=B_{36 a}$ (cf. Table F-2).

Theorem 5.1, together with the explicit calculation, also implies the following:

Corollary 5.3. Let $X$ be a minimal projective 3-fold of general type. Then,

(1) if $\delta(X)=13, P_{m}>0$ for all $m \geq 10$;

(2) if $\delta(X)=14,15,18, P_{m}>0$ for all $m \geq 20$.

\section{Birationality}

Theorem 6.1. Let $X$ be a minimal projective 3-fold of general type. If $\delta(X)=18$, then $\Phi_{m}$ is birational for all $m \geq 61$.

Proof. Set $m_{0}=18$. By Theorem 5.1, we know that $B_{X}=B_{2 a}, P_{2}=0$, $\chi\left(\mathcal{O}_{X}\right)=2, P_{19}=0, P_{24}=3$ and $K_{X}^{3}=\frac{1}{1170}$. By [5, Corollary 1.2], we see $q(X)=0$. Thus $\left|18 K_{X}\right|$ induces a fibration $f: X^{\prime} \longrightarrow \Gamma \cong \mathbb{P}^{1}$. We have $h^{2}\left(\mathcal{O}_{X^{\prime}}\right)=h^{2}\left(\mathcal{O}_{X}\right)=1$. Pick a general fiber $F$. Since $P_{19}(X)=$ $P_{19}\left(\mathbb{B}_{2 a}\right)=0$, we have $H^{0}\left(X^{\prime}, K_{X^{\prime}}+F\right)=0$.

Claim 6.1.1. $p_{g}(F)=1$.

Proof. Since $\chi\left(\mathcal{O}_{X^{\prime}}\right)>1$, we have $p_{g}(F)>0$ by [7, Lemma 2.32]. On the other hand, we have the long exact sequence:

$H^{0}\left(X^{\prime}, K_{X^{\prime}}+F\right) \longrightarrow H^{0}\left(F, K_{F}\right) \longrightarrow H^{1}\left(X^{\prime}, K_{X^{\prime}}\right) \longrightarrow H^{1}\left(X^{\prime}, K_{X^{\prime}}+F\right)$ which implies $h^{0}\left(K_{F}\right) \leq h^{1}\left(X^{\prime}, K_{X^{\prime}}\right)=h^{2}\left(\mathcal{O}_{X^{\prime}}\right)=1$. Thus we get $p_{g}(F)=1$.

We have $P_{m}>0$ for all $m \geq 20$ by Corollary 5.3 (2). Consider the linear systems

$$
\left|K_{X^{\prime}}+\left\lceil n \pi^{*}\left(K_{X}\right)\right\rceil+F\right| \preceq\left|(n+19) K_{X^{\prime}}\right| .
$$

Clearly $\left|(n+19) K_{X^{\prime}}\right|$ distinguish different general fibers $F$ as long as $n \geq 19$. By Kawamata-Viehweg vanishing,

$$
\begin{aligned}
\mid K_{X^{\prime}}+\left\lceil n \pi^{*}\left(K_{X}\right)\right\rceil+F\|\|_{F} & =\left|K_{F}+\left\lceil n \pi^{*}\left(K_{X}\right)\right\rceil\right|_{F} \mid \\
& \succeq\left|K_{F}+\left\lceil L_{n}\right\rceil\right|
\end{aligned}
$$

where we set $L_{n}:=\left.n \pi^{*}\left(K_{X}\right)\right|_{F}$. 
Claim 6.1,2. $L_{n}^{2}>8$ whenever $n \geq 42$.

Proof. Since $p_{g}(F)=1$, we are in Subcase 3.4.1 or Subcase 3.4.3.

Let us consider Subcase 3.4.1 (i.e. $K_{F_{0}}^{2} \geq 2$ ) first. We have

$$
\left(\left.\pi^{*}\left(K_{X}\right)\right|_{F}\right)^{2} \geq \frac{1}{19^{2}} K_{F_{0}}^{2} \geq \frac{2}{19^{2}}
$$

by Lemma 2.1(ii). Thus $L_{n}^{2}>8$ whenever $n>38$.

If $K_{F_{0}}^{2}=1$, we shall estimate $L_{n}^{2}$ in an alternative way. Suppose that $\left|24 K_{X^{\prime}}\right|$ and $\left|18 K_{X^{\prime}}\right|$ are not composed with the same pencil. Take $|G|:=\left|M_{24}\right|_{F} \mid$. Pick a generic irreducible element $C$ of $|G|$. Then we have $\xi=\left(\left.\pi^{*}\left(K_{X}\right)\right|_{F} \cdot C\right) \geq \frac{2}{19}$ by Lemma 2.4. Thus $\left(\left.\pi^{*}\left(K_{X}\right)\right|_{F}\right)^{2} \geq$ $\frac{1}{24} \xi \geq \frac{1}{12 \cdot 19}$. Since $r(X)=2340$ and $r(X)\left(\left.\pi^{*}\left(K_{X}\right)\right|_{F}\right)^{2}$ is an integer, we see $\left(\left.\pi^{*}\left(K_{X}\right)\right|_{F}\right)^{2} \geq \frac{11}{2340}$. So we have $L_{n}^{2}>8$ whenever $n \geq 42$.

Assume that $\left|24 K_{X^{\prime}}\right|$ and $\left|18 K_{X^{\prime}}\right|$ are composed with the same pencil. Since $P_{24}=3$, we may set $m_{0}=24$ and $\Lambda=\left|24 K_{X^{\prime}}\right|$. We have $\theta=2$. The argument in Subcase 3.4.3 implies that

$$
\left(\left.\pi^{*}\left(K_{X}\right)\right|_{F}\right)^{2} \geq \frac{4 \theta^{2}}{\left(\tilde{m}_{0}+\theta\right)\left(3 m_{0}+4 \theta\right)}=\frac{1}{130} .
$$

We have $L_{n}^{2}>8$ whenever $n \geq 33$.

For very general curves $\tilde{C}$ on $F$, one has

$$
\left(L_{n} \cdot \tilde{C}\right) \geq \frac{n}{19}\left(\sigma^{*}\left(K_{F_{0}}\right) \cdot \tilde{C}\right) \geq \frac{2 n}{19}
$$

by Lemma 2.5. Therefore, $\left(L_{n} \cdot \tilde{C}\right) \geq 4$ for $n \geq 38$. Lemma 2.3 implies that $\left|K_{F}+\left\lceil L_{n}\right\rceil\right|$ gives a birational map for $n \geq 42$. Thus $\Phi_{m}$ is birational for all $m \geq 61$.

Theorem 6.2. Let $X$ be a minimal projective 3-fold of general type. If $\delta(X) \leq 15$, then $\Phi_{m}$ is birational for all $m \geq 56$.

Proof. Set $m_{0}=\delta(X)$. By considering a sub-pencil $\Lambda$ of $\left|m_{0} K_{X}\right|$, we may always assume that we have an induced fibration $f: X^{\prime} \longrightarrow \Gamma$ onto a curve $\Gamma$. By Chen-Hacon [9], we may assume $q(X)=0$. Thus $\Gamma \cong \mathbb{P}^{1}$. By [7, Corollary 3.13] and [7, Lemma 2.32], we know that $\delta(X) \leq 10$ as long as $F$ is a $(1,0)$ surface. Therefore it suffices to consider the following 3 cases:

1. $\delta(X) \leq 15$ and $F$ is a $(1,2)$ surface.

2. $\delta(X) \leq 15$ and $F$ is neither a $(1,2)$ surface nor a $(1,0)$ surface.

3. $\delta(X) \leq 10$ and $F$ is a $(1,0)$ surface.

Case 1. Without losing of generality, let us assume $\delta(X)=15$. Take $|G|$ to be the moving part of $\left|K_{F}\right|$. Then, by Table A3, we have $\xi \geq \frac{1}{11}$. We have $m_{0}=15$ and $\beta \mapsto \frac{1}{16}$. So $\alpha_{m}>2$ whenever $m \geq 55$. By Corollary 5.3, $\left|m K_{X^{\prime}}\right|$ separates different general fibers $F$ as long as 
$m \geq 35$. On the other hand, Kawamata-Viehweg vanishing and Lemma 2.1 imply the following, whenever $m \geq 49$,

$$
\begin{aligned}
\mid m K_{X^{\prime}} \|_{F} & \succeq \mid K_{X^{\prime}}+\left\lceil(m-16) \pi^{*}\left(K_{X}\right)\right\rceil+F \|_{F} \\
& \succeq \mid K_{F}+\left\lceil\left.(m-16) \pi^{*}\left(K_{X}\right)\right|_{F}\right\rceil \\
& \succeq\left|\left(K_{F}+\left\lceil Q_{m}\right\rceil+C\right)+C\right|
\end{aligned}
$$

where $Q_{m}$ is a nef and big $\mathbb{Q}$-divisor. Thus, by [7, Lemma 2.17], $\Phi_{m}$ distinguishes different generic curves $C$ for $m \geq 49$. Finally Theorem 2.7 implies that $\Phi_{m}$ is birational for all $m \geq 55$.

Case 2. Still assume $\delta(X)=15$. Parallel to the respective argument in the proof of Theorem 6.1, one knows that $\left|m K_{X^{\prime}}\right|$ distingishes different general fibers $F$ for $m \geq 35$. By the surface theory, we see that $F$ is either a surface with $K_{F_{0}}^{2} \geq 2$ or a $(1,1)$ surface. We want to study the linear system $\left|K_{F}+\left\lceil L_{n}\right\rceil\right|$. In fact, by the estimation in Subcase 3.4. 1 and Table A4, we have $L_{n}^{2} \geq \frac{n^{2}}{32 \cdot 6}>8$ whenever $n \geq 40$. Similarly we have $\left(L_{n} \cdot \tilde{C}\right) \geq 4$ for all $n \geq 32$ and for all curves $\tilde{C}$ on $F$ passing through very general points. By Lemma 2.3, we see that $\left|K_{F}+\left\lceil L_{n}\right\rceil\right|$ gives a birational map for all $n \geq 40$. Similar to what discussed in the proof of Theorem 6.1, we have proved that $\Phi_{m}$ is birational for all $m \geq n+16 \geq 56$.

Case 3. When $\delta(X) \leq 10$, we have much better birationality result even though $F$ is a $(1,0)$ surface. In fact, parallel argument shows that $\Phi_{m}$ is birational for all $m \geq 39$. The proof is more or less similar to above ones. We leave it as an exercise to interested readers.

Theorems 5.1, 6.1, and 6.2 imply Theorem 1.6 (2).

\section{Threefolds with $\delta(V)=2$}

This section is devoted to classifying minimal projective threefolds of general type with $\delta(X)=2$, that is, $p_{g}(X) \leq 1$ and $P_{2}(X) \geq 2$.

Assume that $P_{2} \geq 2$. We first recall the following known results:

(a) If $d_{2}=3$, then $\Phi_{m}$ is birational for all $m \geq 7$ by [7, Theorem 2.20].

(b) If $d_{2}=2, \Phi_{m}$ is birational for all $m \geq 10$ by [7, Theorem 2.22].

(c) If $q(X)>0$, then $\Phi_{m}$ is birational for all $m \geq 7$ by Chen-Hacon [9] and for $m=6$ by Chen-Chen-Jiang [8].

The purpose of this section is to prove that $\Phi_{m}$ is birational for $m \geq$ 11 and classify threefolds such that $\Phi_{10}$ is not birational. Therefore, we may and do assume that $q(X)=0, d_{2}=1$ and $b=g(\Gamma)=0$. Let $F$ be the general fiber of the induced fibration $f: X^{\prime} \rightarrow \mathbb{P}^{1}$ from $\Phi_{2}$. 


\subsection{Birationality of $\Phi_{m}$ for $m \geq 11$.}

Lemma 7.1. $\left|m K_{X^{\prime}}\right|$ distinguishes different general fibers of $f$ for all $m \geq 9$.

Proof. When $p_{g}(F)>0$, by [7, Proposition 2.15 (i)], one has $P_{k}>0$ for $k \geq 7$. Thus, for all $m \geq 9, m K_{X^{\prime}} \geq F$, hence $\left|m K_{X^{\prime}}\right|$ distinguishes different general fibers of $f$.

When $p_{g}(F)=0$, one has $\chi\left(\mathcal{O}_{X}\right) \leq 1$ (cf. [7, Lemma 2.32]). By [7. Lemma 3.2], one has $P_{5} \geq P_{2}>0$. Then clearly $P_{k}>0$ for all $k \geq 5$. Thus, for all $m \geq 7, m K_{X^{\prime}} \geq F$ and hence $\left|m K_{X^{\prime}}\right|$ distinguishes different general fibers of $f$.

Proposition 7.2. Assume $P_{2}(X) \geq 2, q(X)=0, d_{2}=1$ and $F$ is not a $(1,2)$ surface. Then $\Phi_{m}$ is birational for all $m \geq 10$.

Proof. Set $L_{n}:=\left.n \pi^{*}\left(K_{X}\right)\right|_{F}$ which is a nef and big $\mathbb{Q}$-divisor on $F$. Kawamata-Viehweg vanishing gives the following surjective map:

$$
H^{0}\left(X^{\prime}, K_{X^{\prime}}+\left\lceil n \pi^{*}\left(K_{X}\right)\right\rceil+F\right) \longrightarrow H^{0}\left(F, K_{F}+\left.\left\lceil n \pi^{*}\left(K_{X}\right)\right\rceil\right|_{F}\right) .
$$

Together with Lemma 7.1, it is sufficient to prove that $\left|K_{F}+\left\lceil L_{n}\right\rceil\right|$ gives a birational map for $n \geq 7$ because

$$
\left|(n+3) K_{X^{\prime}}\right| \succeq\left|K_{X^{\prime}}+\left\lceil n \pi^{*}\left(K_{X}\right)\right\rceil+F\right| .
$$

Claim 7.2 .1. If $K_{F_{0}}^{2} \geq 2$ or $F_{0}$ is of type $(1,0)$, then $\left|K_{F}+\left\lceil L_{n}\right\rceil\right|$ is birational for $n \geq 7$.

First of all, for any curve $\tilde{C} \subset F$ passing through very general points of $F$, we estimate $\left(L_{n} \cdot \tilde{C}\right)$ for $n \geq 7$. Clearly we have $g(\tilde{C}) \geq 2$. Set $m_{0}=2$ and $\Lambda=\left|2 K_{X^{\prime}}\right|$. By Lemma 2.1 and Lemma 2.5, we have

$$
\left(L_{n} \cdot \tilde{C}\right) \geq 7\left(\left.\pi^{*}\left(K_{X}\right)\right|_{F} \cdot \tilde{C}\right) \geq \frac{7}{3}\left(\sigma^{*}\left(K_{F_{0}}\right) \cdot \tilde{C}\right)>4 .
$$

If $K_{F_{0}}^{2} \geq 2$, then we have

$$
L_{n}^{2} \geq 49\left(\left.\pi^{*}\left(K_{X}\right)\right|_{F}\right)^{2} \geq 49\left(\frac{1}{3} \sigma^{*}\left(K_{F_{0}}\right)\right)^{2} \geq \frac{98}{9}>8 .
$$

If $F_{0}$ is a $(1,0)$ surface, we have $P_{4} \geq 2 P_{2} \geq 4$ since $\chi\left(\mathcal{O}_{X}\right) \leq 1$. When $d_{4} \geq 2$, we set $m_{0}=2, \Lambda=\left|2 K_{X^{\prime}}\right|$ and $|G|=\left|M_{4}\right|_{F} \mid$. Then $\beta=\frac{1}{4}, \xi \geq \frac{1}{3}\left(\sigma^{*}\left(K_{F_{0}}\right) \cdot C\right) \geq \frac{2}{3}$ and so $L_{n}^{2} \geq \frac{49}{6}>8$.

When $d_{4}=1$, we set $m_{0}=4$ and $\Lambda=\left|4 K_{X^{\prime}}\right|$. Clearly $\left|2 K_{X^{\prime}}\right|$ and $\left|4 K_{X^{\prime}}\right|$ induce the same fibration $f$. Take $|G|=\left|2 \sigma^{*}\left(K_{F_{0}}\right)\right|$. Since $\theta \geq$ 3 , we have $\beta \geq \frac{3}{14}$ by Lemma 2.1. Thus $\xi \geq \frac{6}{7}$ and so $L_{n}^{2} \geq 49 \cdot \frac{3}{14} \cdot \frac{6}{7}>8$. By Lemma 2.3, the Claim follows.

Claim $\left\lceil .2\right.$.2. If $F_{0}$ is a $(1,1)$ surface, then $\left|K_{F}+\left\lceil L_{n}\right\rceil\right|$ is birational for $n \geq 7$.

Following the similar argument as above, it is easy to see that $L_{n}^{2} \geq$ $\frac{64}{7}>8$ and $\left(L_{n} \cdot \tilde{C}\right) \geq 4$ for all $n \geq 8$. We consider the linear system $\left|K_{F}+\left\lceil\left. 7 \pi^{*}\left(K_{X}\right)\right|_{F}\right\rceil\right|$ in an alternative way. Note that $\left.\mid 2 \sigma^{*}\left(K_{F_{0}}\right)\right) \mid$ is 
base point free. Pick a generic irreducible element $\left.C \in \mid 2 \sigma^{*}\left(K_{F_{0}}\right)\right) \mid$. Since $\mathcal{O}_{\Gamma}(1) \hookrightarrow f_{*} \omega_{X^{\prime}}$, we have $f_{*} \omega_{X^{\prime} / \Gamma}^{2} \hookrightarrow f_{*} \omega_{X^{\prime}}^{10}$. The semi-positivity implies that $f_{*} \omega_{X^{\prime} / \Gamma}^{2}$ is generated by global sections, which directly implies $\left.10 K_{X^{\prime}}\right|_{F} \geq C$. Thus $\Phi_{10}$ distinguishes different $C$. By Lemma 2.1, we have $\left.6 \pi^{*}\left(K_{X}\right)\right|_{F} \equiv C+H_{6}$ for an effective $\mathbb{Q}$-divisor $H_{6}$ on $F$. Thus the vanishing theorem implies

$$
\left.\left|K_{F}+\left\lceil\left. 7 \pi^{*}\left(K_{X}\right)\right|_{F}-H_{6}\right\rceil\right|\right|_{C}=\left|K_{C}+D\right|
$$

with $\operatorname{deg}(D) \geq 2\left(\left\lceil\left. 7 \pi^{*}\left(K_{X}\right)\right|_{F}-C-H_{6}\right\rceil \cdot \sigma^{*}\left(K_{F_{0}}\right)\right) \geq 2$. Since $C$ is non-hyperelliptic, $\left|K_{C}+D\right|$ gives a birational map. Thus $\mid K_{F}+$ $\left\lceil\left. 7 \pi^{*}\left(K_{X}\right)\right|_{F}\right\rceil \mid$ is birational.

Proposition 7.3. Assume $P_{2}(X) \geq 2, q(X)=0, d_{2}=1$ and $F a$ $(1,2)$ surface. Then $\Phi_{m}$ is birational for all $m \geq 11$.

Proof. Take $|G|$ to be the moving part of $\left|\sigma^{*}\left(K_{F_{0}}\right)\right|$. Modulo birational modifications, we may assume that $|G|$ is base point free. Pick a generic irreducible element $C$ of $|G|$. It is also known that $g=2$.

Claim 7.3.1 The linear system $\left|m K_{X^{\prime}}\right|$ distinguishes different general members of $|G|$ for $m \geq 9$.

Proof. Clearly $|G|$ is composed with a rational pencil since $q(F)=0$. We shall prove $\left|m K_{X^{\prime}}\right|_{\mid F} \succeq|G|$ and thus the statement follows. In fact, by Lemma 2.1, we have

$$
3 \pi^{*}\left(K_{X}\right) \equiv \sigma^{*}\left(K_{F_{0}}\right)+H_{3}
$$

for an effective $\mathbb{Q}$-divisor $H_{3}$ on $F$. Thus, for $m \geq 10$,

$$
Q_{m}:=(m-3) \pi^{*}\left(K_{X}\right)_{\mid F}-2 H_{3}-\left.2 \sigma^{*}\left(K_{F_{0}}\right) \equiv(m-9) \pi^{*}\left(K_{X}\right)\right|_{F}
$$

is nef and big. It follows that $K_{F}+\left\lceil Q_{m}\right\rceil+\sigma^{*}\left(K_{F_{0}}\right)>0$ by [7, Lemma 2.14]. We thus have the following:

$$
\begin{aligned}
\left|m K_{X^{\prime}}\right|_{\mid F} & \succeq\left|K_{X^{\prime}}+F+\left\lceil(m-3) \pi^{*}\left(K_{X}\right)\right\rceil\right|_{\mid F} \\
& =\left|K_{F}+\left\lceil(m-3) \pi^{*}\left(K_{X}\right)\right\rceil_{\mid F}\right| \\
& \succeq\left|K_{F}+\left\lceil(m-3) \pi^{*}\left(K_{X}\right)_{\mid F}-2 H_{3}\right\rceil\right| \\
& =\left|\left(K_{F}+\left\lceil Q_{m}\right\rceil+\sigma^{*}\left(K_{F_{0}}\right)\right)+\sigma^{*}\left(K_{F_{0}}\right)\right| \\
& \succeq\left|\sigma^{*}\left(K_{F_{0}}\right)\right| \succeq|G|
\end{aligned}
$$

where the first equality follows from the Kawamata-Viehweg vanishing ([19, 33]). Therefore, $\left|m K_{X^{\prime}}\right|$ distinguishes general members of $|G|$ for $m \geq 10$. Moreover, for $m=9$,

$$
\begin{aligned}
\left|9 K_{X^{\prime}}\right|_{\mid F} & \succeq\left|5 K_{X^{\prime}}\right|_{\mid F} \succeq\left|K_{X^{\prime}}+\left\lceil 2 \pi^{*}\left(K_{X}\right)\right\rceil+F\right|_{\mid F} \\
& =\left|K_{F}+\left\lceil 2 \pi^{*}\left(K_{X}\right)\right\rceil\right|_{F}|\succeq| G \mid
\end{aligned}
$$

where the equality is again due to Kawamata-Viehweg vanishing. Hence $\left|9 K_{X^{\prime}}\right|$ distinguishes general members of $|G|$ as well, which asserts the claim. 
From Table A3, one has $\xi \geq \frac{1}{2}$. Take $m \geq 11$, then $\alpha_{m} \geq \frac{5}{2}>2$. This means that $\left|m K_{X^{\prime}}\right|_{\mid C}$ distinguishes points on $C$. Thus, by Theorem 2.7 and Claim 7.3.1, $\Phi_{m}$ is birational for all $m \geq 11$.

Now Theorem 1.8. 1 follows from Proposition 7.2 and Proposition 7.3. That is, if $P_{2} \geq 2$, then $\Phi_{m}$ is birational for $m \geq 11$.

If either $\xi>\frac{1}{2}$ or $\beta>\frac{1}{3}$ then $\alpha_{10}>2$. Hence the following consequence is immediate.

Corollary 7.4. Let $X$ be a minimal projective 3-fold of general type. Assume $P_{2}(X) \geq 2, q(X)=0, d_{2}=1$ and $F_{0}$ a $(1,2)$ surface. If either $\xi>\frac{1}{2}$ or $\beta>\frac{1}{3}$ or $P_{2}>2$, then $\Phi_{10}$ is birational.

Proposition [7.2, Proposition 7.3 and Corollary 7.4 also imply the following:

Corollary 7.5. Let $X$ be a minimal projective 3-fold of general type. Assume $P_{2} \geq 2$ and $\Phi_{10}$ is not birational. Then $P_{2}=2, q(X)=0$ and $\left|2 K_{X^{\prime}}\right|$ is composed with a rational pencil of $(1,2)$ surfaces.

7.2. Classification. In the rest of this section, we classify minimal 3-folds $X$ of general type which satisfy the following assumptions:

$(\sharp) P_{2}(X)=2$ and $\Phi_{10}$ is not birational.

Note that Corollary 7.5 implies that $\left|2 K_{X}\right|$ induces a fibration $f$ : $X^{\prime} \longrightarrow \mathbb{P}^{1}$ with the general fiber $F$ a $(1,2)$ surface.

Lemma 7.6. If $X$ satisfies $(\sharp)$, then $0 \leq \chi\left(\mathcal{O}_{X}\right) \leq 3$.

Proof. Note that the general fiber $F$ of $f$ is a $(1,2)$ surface. Since $q(F)=0$, we have $q(X)=0, h^{2}\left(\mathcal{O}_{X}\right)=h^{1}\left(\mathbb{P}^{1}, f_{*} \omega_{X^{\prime}}\right)$ and $p_{g}(X)=$ $h^{0}\left(f_{*} \omega_{X^{\prime}}\right)$. Since $P_{2}(X)=2$ implies $p_{g}(X) \leq 1$, we see $\chi\left(\mathcal{O}_{X}\right) \geq 0$. By Fujita's semi-positivity $([16])$, we have $\chi\left(\mathcal{O}_{X}\right) \leq 3$.

Theorem 7.7. Let $X$ be a minimal projective 3-fold of general type. Assume $P_{2}=2, q(X)=0$ and $F$ a $(1,2)$ surface. Then $\Phi_{10}$ is birational under one of the following conditions:

(1) $P_{3} \geq 4$

(2) $P_{4} \geq 6$

(3) $P_{5} \geq 8$;

(4) $P_{6} \geq 14$

Proof. We set $m_{0}=2$. Pick a general fiber $F$ of $f: X^{\prime} \longrightarrow \Gamma$ and a generic irreducible element $C$ of $|G|:=\operatorname{Mov}\left|\sigma^{*}\left(K_{F_{0}}\right)\right|$ on $F$. For $m_{1}=$ $3,4,5$ and 6 , we have $P_{m_{1}} \geq 4$. Modulo further birational modifications to $\pi$, we may assume that the moving part $\left|M_{m_{1}}\right|$ of $\left|m_{1} K_{X^{\prime}}\right|$ is base point free. We consider the following natural maps:

$$
H^{0}\left(X^{\prime}, S_{m_{1}}\right) \stackrel{\mu_{m_{1}}}{\longrightarrow} H^{0}\left(F,\left.S_{m_{1}}\right|_{F}\right) \stackrel{\nu_{m_{1}}}{\longrightarrow} H^{0}\left(C,\left.S_{m_{1}}\right|_{C}\right)
$$

where $S_{m_{1}} \in\left|M_{m_{1}}\right|$ denotes the general member. 
Let Mov $\left|S_{m_{1}}\right|_{F} \mid$ be the moving part of $\left|S_{m_{1}}\right|_{F} \mid$ and let $T_{m_{1}}$ be a general element in Mov $\left|S_{m_{1}}\right|_{F} \mid$ when $h^{0}\left(F,\left.S_{m_{1}}\right|_{F}\right)>1$. Clearly

$$
\left(S_{m_{1}} \cdot C\right)_{X^{\prime}} \geq\left(T_{m_{1}} \cdot C\right)_{F} \geq 0 .
$$

Since $F$ and $C$ are general, both $\mu_{m_{1}}$ and $\nu_{m_{1}}$ are non-zero maps. In particular, $h^{0}\left(F,\left.S_{m_{1}}\right|_{F}\right)>0$ and $h^{0}\left(C,\left.S_{m_{1}}\right|_{C}\right)>0$.

Let $F_{(r)}$ be a general element in Mov $\left|S_{m_{1}}-r F\right|$ if $h^{0}\left(S_{m_{1}}-r F\right) \geq 2$. Let $C_{(r)}$ be a general element in Mov $\left|T_{m_{1}}-r C\right|$ if $h^{0}\left(T_{m_{1}}-r C\right) \geq 2$. Replace $X^{\prime}$ by its birational modification, we may and do assume that $\operatorname{Mov}\left|S_{m_{1}}-r F\right|$ is free.

Clearly, for $0<r \leq \frac{h^{0}\left(X^{\prime}, S_{m_{1}}\right)}{h^{0}\left(F, S_{m_{1}} \mid F\right)}$, we have

$$
h^{0}\left(X^{\prime}, S_{m_{1}}-r F\right) \geq h^{0}\left(X^{\prime}, S_{m_{1}}\right)-r \cdot h^{0}\left(F,\left.S_{m_{1}}\right|_{F}\right) .
$$

Claim 7.7.1. If $\left(T_{m_{1}} \cdot C\right) \leq 1$, then $\left(T_{m_{1}} \cdot C\right)=0$.

Proof. In fact, if $\left|T_{m_{1}}\right| \neq \emptyset$ and $\left|T_{m_{1}}\right|$ is not composed of the same pencil as that of $|C|$, then $\Phi_{\left|T_{m_{1}}\right|}(C)$ is a curve and so $h^{0}\left(C,\left.T_{m_{1}}\right|_{C}\right) \geq 2$. Note that $g(C)=2$. The Riemann-Roch theorem and the Clifford theorem imply that $\left(T_{m_{1}} \cdot C\right)=\operatorname{deg}\left(\left.T_{m_{1}}\right|_{C}\right) \geq 2$, a contradiction. Hence either $\left|T_{m_{1}}\right|$ is composed of the same pencil as that of $|C|$ on $F$ or $\left|T_{m_{1}}\right|=\emptyset$. Claim 7.7,1 now follows.

Claim 7.7.2. Keep the same notation as above. Then $\Phi_{10}$ is birational under one of the following conditions:

(1) $\left(T_{m_{1}} \cdot C\right)>\frac{m_{1}}{2}$;

(2) $T_{m_{1}} \cdot C=0$ and $h^{0}\left(F, T_{m_{1}}\right)>1+\frac{m_{1}}{3}$;

(3) $T_{m_{1}} \geq t C$ for some rational number $t>\frac{m_{1}}{3}$;

(4) either $\left|T_{m_{1}}\right|=\emptyset$ and $P_{m_{1}}>1+\frac{m_{1}}{2}$ or $\left|T_{m_{1}}\right| \neq \emptyset$ and $\left\lfloor\frac{P_{m_{1}}-1}{h^{0}\left(F, T_{m_{1}}\right)}\right\rfloor>$ 5) $\frac{m_{1}}{2}$.

(5) $F_{(r)}\left(\right.$ resp. $\left.C_{(r)}\right)$ is algebraically equivalent to $F$ (resp. C) and $\frac{r+1}{m_{1}}>\frac{1}{2}$ (resp. $\left.\frac{r+1}{m_{1}}>\frac{1}{3}\right)$.

Proof. If $\left(T_{m_{1}} \cdot C\right)>\frac{m_{1}}{2}$, then $\xi \geq \frac{1}{m_{1}}\left(S_{m_{1}} \cdot C\right) \geq \frac{1}{m_{1}}\left(T_{m_{1}} \cdot C\right)>\frac{1}{2}$. Then Corollary 7.4 implies that $\Phi_{10}$ is birational, which proves (1).

Now we prove (4). We claim that we have

$$
m_{1} \pi^{*}\left(K_{X}\right) \geq S_{m_{1}} \geq r F
$$

for an integer $r>\frac{m_{1}}{2}$. In fact, when $\left|T_{m_{1}}\right|=\emptyset,\left|S_{m_{1}}\right|$ is composed of the same pencil as that of $|F|$ and we may take $r:=P_{m_{1}}-1$. When $\left|T_{m_{1}}\right| \neq \emptyset$, we may take $r=\left\lfloor\frac{P_{m_{1}}-1}{h^{0}\left(F, T_{m_{1}}\right)}\right\rfloor$ and then $S_{m_{1}} \geq r F$ since $\operatorname{dim} \operatorname{im}\left(\mu_{m_{1}}\right) \leq h^{0}\left(F, T_{m_{1}}\right)$. Then Lemma 2.1 implies $\beta \geq \frac{r}{m_{1}+r}>\frac{1}{3}$. So $\Phi_{10}$ is birational by Corollary [7.4, which asserts (4).

Since $\left.m_{1} \pi^{*}\left(K_{X}\right)\right|_{F} \geq T_{m_{1}} \geq t C$, we have $\beta>\frac{1}{3}$ and $\Phi_{10}$ is birational by Corollary 7.4 , which proves (3). 
If $\left(T_{m_{1}} \cdot C\right)=0$ and $h^{0}\left(F, T_{m_{1}}\right)>1+\frac{m_{1}}{3}$, then $\left|T_{m_{1}}\right|$ is composed of the same pencil as that of $|C|$ and $T_{m_{1}} \geq t C$ where $t \geq h^{0}\left(T_{m_{1}}\right)-1$. Hence $\Phi_{10}$ is birational by (3), which proves (2).

Finally, if $F_{(r)}$ is algebraically equivalent to $F$, then $S_{m_{1}} \geq F_{(r)}+F \sim$ $(r+1) F$. Hence $\beta \geq \frac{r+1}{m_{1}+r+1}>\frac{1}{3}$. Thus $\Phi_{10}$ is birational by Corollary 7.4. If $C_{(r)}$ is algebraically equivalent to $C$, then we have $\beta \geq \frac{r+1}{m_{1}}>\frac{1}{3}$ as well. Hence $\Phi_{10}$ is birational, which verifies (5).

Return to the proof of Theorem 7.7 .

Part I. $P_{3} \geq 4$. Set $m_{1}=3$. By Claim 7.7.2 (1), (2) and Claim 7.7,1, we may assume $\left(T_{3} \cdot C\right)=0$ and $h^{0}\left(F, T_{3}\right) \leq 2$. Also by Claim [7.7,2 (4), we may assume $\left|T_{3}\right| \neq \emptyset$ and $h^{0}\left(F, T_{3}\right)=2$.

By Inequality (17.1), one gets $h^{0}\left(S_{3}-F\right) \geq 2$. Clearly we have that $S_{3} \geq F+F_{(1)}$ and that, by assumption, $F_{(1)}$ is nef. Since $r=1$ and $\frac{r+1}{m_{1}}=\frac{2}{3}>\frac{1}{2}$, we may assume that $F_{(1)}$ is not algebraically equivalent to $F$ by Claim 7.7.2 (5).

Now clearly we have $h^{0}\left(F,\left.F_{(1)}\right|_{F}\right) \geq 2$. Note that we have

$$
\left|10 K_{X^{\prime}}\right| \succeq\left|K_{X^{\prime}}+\left\lceil 6 \pi^{*}\left(K_{X}\right)\right\rceil+F_{(1)}+F\right| .
$$

Kawamata-Viehweg vanishing gives the surjective map:

$$
\begin{aligned}
& H^{0}\left(X^{\prime}, K_{X^{\prime}}+\left\lceil 6 \pi^{*}\left(K_{X}\right)\right\rceil+F_{(1)}+F\right) \\
\longrightarrow & H^{0}\left(F, K_{F}+\left.\left\lceil 6 \pi^{*}\left(K_{X}\right)\right\rceil\right|_{F}+\left.F_{(1)}\right|_{F}\right) .
\end{aligned}
$$

It is sufficient to verify the birationality of the rational map defined by $\left|K_{F}+\left\lceil\left. 6 \pi^{*}\left(K_{X}\right)\right|_{F}\right\rceil+\Gamma_{(1)}\right|$ where $\Gamma_{(1)}$ is a generic irreducible element in $\mathrm{Mov}\left|F_{(1)}\right| F \mid$.

We claim that $\left(\pi^{*}\left(K_{X}\right) \cdot \Gamma_{(1)}\right) \geq \frac{1}{2}$. In fact, if $\Gamma_{(1)}$ is algebraically equivalent to $C$, then $\left(\pi^{*}\left(K_{X}\right) \cdot \Gamma_{(1)}\right)=\xi \geq \frac{1}{2}$ by Table A3. On the other hand, if $\Gamma_{(1)}$ is not algebraically equivalent to $C$, then we should have $\left(\Gamma_{(1)} \cdot C\right) \geq 2$. By Lemma 2.1, $\left(\left.\pi^{*}\left(K_{X}\right)\right|_{F} \cdot \Gamma_{(1)}\right) \geq \frac{1}{3}\left(C \cdot \Gamma_{(1)}\right) \geq \frac{2}{3}$.

Clearly $\left|K_{F}+\left\lceil\left. 6 \pi^{*}\left(K_{X}\right)\right|_{F}\right\rceil+\Gamma_{(1)}\right|$ distinguishes different generic $\Gamma_{(1)}$ 's since $K_{F}+\left\lceil\left. 6 \pi^{*}\left(K_{X}\right)\right|_{F}\right\rceil>0$. Now by the vanishing theorem again we have the following surjective map:

$$
H^{0}\left(F, K_{F}+\left\lceil\left. 6 \pi^{*}\left(K_{X}\right)\right|_{F}\right\rceil+\Gamma_{(1)}\right) \longrightarrow H^{0}\left(\Gamma_{(1)}, K_{\Gamma_{(1)}}+D\right)
$$

where $D:=\left.\left\lceil\left. 6 \pi^{*}\left(K_{X}\right)\right|_{F}\right\rceil\right|_{\Gamma_{(1)}}$ with $\operatorname{deg}(D) \geq 6\left(\pi^{*}\left(K_{X}\right) \cdot \Gamma_{(1)}\right)>2$. So $\Phi_{10}$ is birational by the ordinary birationality principle.

Part II. $P_{4} \geq 6$. We set $m_{1}=4$. By Claim 7.7,2 (1) and (4), we may assume $\left(T_{4} \cdot C\right) \leq 2$ and $h^{0}\left(F, T_{4}\right) \geq 2$. Claim 7.7.1 implies either $\left(T_{4} \cdot C\right)=0$ or $\left(T_{4} \cdot C\right)=2$.

(II-1). If $h^{0}\left(F, T_{4}\right)=2$, we have $h^{0}\left(X^{\prime}, S_{4}-2 F\right) \geq 2$ by Inequality (7.1). We consider $F_{(2)}$ and may assume that $F_{(2)}$ is not algebraically equivalent to $F$ by Claim $7.7,2(5)$. Now $h^{0}\left(F, F_{(2)} \mid F\right) \geq 2$ and pick a 
generic irreducible element $\Gamma_{(2)}$ of Mov $\left|F_{(2)}\right|_{F} \mid$. By Kawamata-Viehweg vanishing, we have

$$
\begin{aligned}
\mid 10 K_{X^{\prime}} \|_{F} & \succeq\left|K_{X^{\prime}}+\left\lceil 5 \pi^{*}\left(K_{X}\right)\right\rceil+F_{(2)}+2 F\right| \|_{F} \\
& =\left|K_{F}+\left\lceil 5 \pi^{*}\left(K_{X}\right)\right\rceil\right|_{F}+\left.F_{(2)}\right|_{F} \mid \\
& \succeq\left|K_{F}+\left\lceil\left. 5 \pi^{*}\left(K_{X}\right)\right|_{F}\right\rceil+\Gamma_{(2)}\right| .
\end{aligned}
$$

When $C$ is algebraically equivalent to $\Gamma_{(2)}$ (in particular, $C \sim \Gamma_{(2)}$ due to the fact that $q(F)=0$ ), since

$$
\operatorname{deg}\left(\left.5 \pi^{*}\left(K_{X}\right)\right|_{C}\right)=5 \xi \geq \frac{5}{2}
$$

and

$$
\left.\left|K_{F}+\left\lceil\left. 5 \pi^{*}\left(K_{X}\right)\right|_{F}\right\rceil+\Gamma_{(2)}\right|\right|_{C}=\left|K_{C}+\left\lceil\left. 5 \pi^{*}\left(K_{X}\right)\right|_{F}\right\rceil\right|_{C} \mid
$$

with $\operatorname{deg}\left(\left.\left\lceil\left. 5 \pi^{*}\left(K_{X}\right)\right|_{F}\right\rceil\right|_{C}\right)>2$, we see that $\left.\Phi_{10}\right|_{C}$ is birational by Lemma 7.1 and Claim 7.3.1.

When $C$ is not algebraically equivalent to $\Gamma_{(2)}$, we have $\left(\Gamma_{(2)} \cdot C\right) \geq 2$ and

$$
K_{F}+\left\lceil\left. 5 \pi^{*}\left(K_{X}\right)\right|_{F}\right\rceil+\Gamma_{(2)} \geq K_{F}+\left\lceil Q_{1}+C\right\rceil+\Gamma_{(2)}
$$

for certain nef and big $\mathbb{Q}$-divisor $Q_{1}$ on $F$ by Lemma 2.1. The vanishing theorem also shows that

$$
\left|K_{F}+\left\lceil Q_{1}\right\rceil+\Gamma_{(2)}+C\right|_{C}=\left|K_{C}+\left(Q_{1}+\Gamma_{(2)}\right)\right|_{C} \mid
$$

gives a birational map since $\operatorname{deg}\left(\left.\left(Q_{1}+\Gamma_{(2)}\right)\right|_{C}\right)>2$. Thus we have shown that $\Phi_{10}$ is birational by Lemma 7.1 and Claim 7.3.1.

(II-2). If $\left(T_{4} \cdot C\right)=0$ and $h^{0}\left(F, T_{4}\right) \geq 3, \Phi_{10}$ is birational by Claim 7.7.2 (2).

(II-3). If $\left(T_{4} \cdot C\right)=2$ and $h^{0}\left(F, T_{4}\right) \geq 3$, then $\left|T_{4}\right|$ is not composed of the same pencil as that of $|C|$ and $h^{0}\left(C,\left.T_{4}\right|_{C}\right) \geq 2$. By the RiemannRoch and the Clifford theorem, we see $\operatorname{deg}\left(\left.T_{4}\right|_{C}\right)=h^{0}\left(C,\left.T_{4}\right|_{C}\right)=2$. Thus $\operatorname{dimim}\left(\nu_{4}\right)=2$.

(II-3-1). If $h^{0}\left(F, T_{4}\right) \geq 4$, we have $h^{0}\left(F, T_{4}-C\right) \geq 2$. Denote by $C_{(1)}$ a generic irreducible element of $\mathrm{Mov}\left|T_{4}-C\right|$. Then we have $T_{4} \geq C+C_{(1)}$ and we may assume that $C$ is not algebraically equivalent to $C_{(1)}$ by Claim [7.7,2 (5), which implies $\left(C_{(1)} \cdot C\right) \geq 2$. By the KawamataViehweg vanishing and properties of the roundup operator, we have

$$
\begin{aligned}
\mid 10 K_{X^{\prime}} \|_{F} & \left.\succeq\left|K_{X^{\prime}}+\left\lceil 3 \pi^{*}\left(K_{X}\right)\right\rceil+S_{4}+F\right|\right|_{F} \\
& =\left|K_{F}+\left\lceil 3 \pi^{*}\left(K_{X}\right)\right\rceil\right|_{F}+\left.S_{4}\right|_{F} \mid \\
& \succeq\left|K_{F}+\left\lceil\left. 3 \pi^{*}\left(K_{X}\right)\right|_{F}\right\rceil+C_{(1)}+C\right|
\end{aligned}
$$

and

$$
\left|K_{F}+\left\lceil\left. 3 \pi^{*}\left(K_{X}\right)\right|_{F}\right\rceil+C_{(1)}+C\right|_{C}=\left|K_{C}+D\right|,
$$

where $D:=\left.\left(\left\lceil\left. 3 \pi^{*}\left(K_{X}\right)\right|_{F}\right\rceil+C_{(1)}\right)\right|_{C}$ with $\operatorname{deg}(D)>\left(C_{(1)} \cdot C\right) \geq 2$. Thus $\Phi_{10}$ is birational by Lemma 7.1 and Claim [7.3.1. 
(II-3-2). If $h^{0}\left(F, T_{4}\right)=3$, we have $h^{0}\left(S_{4}-F\right) \geq 3$. Again, we pick a general member $F_{(1)} \in \operatorname{Mov}\left|S_{4}-F\right|$. Consider the natural map:

$$
H^{0}\left(X^{\prime}, F_{(1)}\right) \stackrel{\mu_{4}^{\prime}}{\longrightarrow} H^{0}\left(F,\left.F_{(1)}\right|_{F}\right) \subset H^{0}\left(F,\left.S_{4}\right|_{F}\right) .
$$

When $\operatorname{dim} \operatorname{im}\left(\mu_{4}^{\prime}\right)=3$, we see $\operatorname{dim} \nu_{4}\left(\operatorname{im}\left(\mu_{4}^{\prime}\right)\right)=\operatorname{dim} \nu_{4}\left(\operatorname{im}\left(\mu_{4}\right)\right)=2$; when $\operatorname{dim} \operatorname{im}\left(\mu_{4}^{\prime}\right)=2$, we consider the situation $\operatorname{dim} \nu_{4}\left(\operatorname{im}\left(\mu_{4}^{\prime}\right)\right) \leq 1$ at first. In both cases, $h^{0}\left(F,\left.F_{(1)}\right|_{F}-C\right)>0$ and thus $\left.F_{(1)}\right|_{F}-C \geq 0$. By the vanishing theorem once more, we have

$$
\begin{aligned}
\mid 10 K_{X^{\prime}} \|_{F} & \succeq \mid K_{X^{\prime}}+\left\lceil 5 \pi^{*}\left(K_{X}\right)\right\rceil+F_{(1)}+F \|_{F} \\
& =\left|K_{F}+\left\lceil 5 \pi^{*}\left(K_{X}\right)\right\rceil\right|_{F}+\left.F_{(1)}\right|_{F} \mid \\
& \succeq\left|K_{F}+\left\lceil\left. 5 \pi^{*}\left(K_{X}\right)\right|_{F}\right\rceil+C\right| .
\end{aligned}
$$

Applying the vanishing theorem again, we see

$$
\left|K_{F}+\left\lceil\left. 5 \pi^{*}\left(K_{X}\right)\right|_{F}\right\rceil+C \|_{C}=\right| K_{C}+D \mid,
$$

where $D:=\left.\left(\left\lceil\left. 5 \pi^{*}\left(K_{X}\right)\right|_{F}\right\rceil\right)\right|_{C}$ with $\operatorname{deg}(D) \geq 5 \xi>2$. Thus $\Phi_{10}$ is birational by Lemma 7.1 and Claim [7.3.1.

When $\operatorname{dim} \operatorname{im}\left(\mu_{4}^{\prime}\right)=\operatorname{dim} \nu_{4}\left(\operatorname{im}\left(\mu_{4}^{\prime}\right)\right)=2$, then $\left|F_{(1)}\right|_{F} \mid$ is not composed with the same pencil as that of $|C|$. In particular, $\left(F_{(1)} \cdot C\right) \geq 2$. By Lemma 2.1, we have

$$
K_{F}+\left\lceil\left. 5 \pi^{*}\left(K_{X}\right)\right|_{F}\right\rceil+\left.F_{(1)}\right|_{F} \geq K_{F}+\left\lceil Q_{2}+C\right\rceil+\left.F_{(1)}\right|_{F}
$$

for certain nef and big $\mathbb{Q}$-divisor $Q_{2}$. Since the vanishing theorem gives

$$
\left|K_{F}+\left\lceil Q_{2}\right\rceil+F_{(1)}\right|_{F}+C \|_{C}=\left|K_{C}+D^{\prime}\right|
$$

with $\operatorname{deg}\left(D^{\prime}\right)>\left(F_{(1)} \cdot C\right) \geq 2$, we see $\Phi_{10}$ is birational too by Lemma 7.1 and Claim 7.3.1.

Consider the last case $\operatorname{dim} \operatorname{im}\left(\mu_{4}^{\prime}\right)=1$. We see that $\left|F_{(1)}\right|$ is composed of the same pencil as that of $|F|$ and $F_{(1)} \geq 2 F$. Thus $S_{4} \geq 3 F$ and, since $\frac{3}{m_{1}}>\frac{1}{2}, \Phi_{10}$ is birational by Claim $7.7,2(5)$.

Part III. $P_{5} \geq 8$. We set $m_{1}=5$. By Claim [7.7.1 and Claim 7.7.2 (1), (2) and (4), we may assume $\left(T_{5} \cdot C\right)=2$ and $h^{0}\left(F, T_{5}\right) \geq 3$. Clearly $\left|T_{5}\right|$ is not composed of the same pencil as that of $|C|$ and so that $h^{0}\left(C,\left.T_{5}\right|_{C}\right) \geq 2$. By the Riemann-Roch and the Clifford theorem, we see $\operatorname{deg}\left(\left.T_{5}\right|_{C}\right)=h^{0}\left(C,\left.T_{5}\right|_{C}\right)=2$. Thus $\operatorname{dim} \operatorname{im}\left(\nu_{5}\right)=2$.

(III-1). If $h^{0}\left(F, T_{5}\right) \geq 4$, we have $h^{0}\left(F, T_{5}-C\right) \geq 2$. Let $C_{(1)}$ be a generic irreducible element in $\operatorname{Mov}\left|T_{5}-C\right|$. Thus we have $T_{5} \geq$ $C+C_{(1)}$ and we may assume that $C_{(1)}$ is not algebraically equivalent to $C$ by Claim 7.7.2 (5). Hence $\left(C_{(1)} \cdot C\right) \geq 2$. By the KawamataViehweg vanishing and properties of the roundup operator, we have the following:

$$
\begin{aligned}
\mid 10 K_{X^{\prime}} \|_{F} & \succeq \mid K_{X^{\prime}}+\left\lceil 2 \pi^{*}\left(K_{X}\right)\right\rceil+S_{5}+F \|_{F} \\
& =\left|K_{F}+\left\lceil 2 \pi^{*}\left(K_{X}\right)\right\rceil\right|_{F}+\left.S_{5}\right|_{F} \mid \\
& \succeq\left|K_{F}+\left\lceil\left. 2 \pi^{*}\left(K_{X}\right)\right|_{F}\right\rceil+C_{(1)}+C\right|
\end{aligned}
$$


and $\left|K_{F}+\left\lceil\left. 2 \pi^{*}\left(K_{X}\right)\right|_{F}\right\rceil+C_{(1)}+C \|_{C}=\right| K_{C}+D \mid$, with

$$
\operatorname{deg}(D)>\left(C_{(1)} \cdot C\right) \geq 2 .
$$

Thus $\Phi_{10}$ is birational by Lemma 7.1 and Claim 7.3.1.

(III-2). If $h^{0}\left(F, T_{5}\right)=3$, we have $h^{0}\left(S_{5}-F\right) \geq 5$. Let $F_{(1)} \in \operatorname{Mov} \mid S_{5}-$ $F \mid$ be a general member. We consider the natural map:

$$
H^{0}\left(X^{\prime}, F_{(1)}\right) \stackrel{\mu_{5}^{\prime}}{\longrightarrow} H^{0}\left(F,\left.F_{(1)}\right|_{F}\right) \subset H^{0}\left(F,\left.S_{5}\right|_{F}\right) .
$$

Clearly we have $\operatorname{dim} \operatorname{im}\left(\mu_{5}^{\prime}\right) \leq h^{0}\left(F, T_{5}\right)=3$.

When $\operatorname{dim} \operatorname{im}\left(\mu_{5}^{\prime}\right)=3$, we see $\operatorname{dim} \nu_{5}\left(\operatorname{im}\left(\mu_{5}^{\prime}\right)\right)=\operatorname{dim} \nu_{5}\left(\operatorname{im}\left(\mu_{5}\right)\right)=2$. Thus $\left|F_{(1)}\right|_{F} \mid$ is not composed of the same pencil as that of $|C|$. Pick a generic irreducible element $\Gamma_{(1)}$ in the moving part of $\left|F_{(1)}\right|{ }_{F} \mid$. Then $\left(\Gamma_{(1)} \cdot C\right) \geq 2$. By the vanishing theorem, we have

$$
\begin{aligned}
\mid 10 K_{X^{\prime}} \|_{F} & \succeq \mid K_{X^{\prime}}+\left\lceil 4 \pi^{*}\left(K_{X}\right)\right\rceil+F_{(1)}+F \|_{F} \\
& =\left|K_{F}+\left\lceil 4 \pi^{*}\left(K_{X}\right)\right\rceil\right|_{F}+\left.F_{(1)}\right|_{F} \mid \\
& \succeq\left|K_{F}+\left\lceil\left. 4 \pi^{*}\left(K_{X}\right)\right|_{F}\right\rceil+\Gamma_{(1)}\right| .
\end{aligned}
$$

Applying Lemma 2.1, we have

$$
\left|K_{F}+\left\lceil\left. 4 \pi^{*}\left(K_{X}\right)\right|_{F}\right\rceil+\Gamma_{(1)}\right| \succeq\left|K_{F}+\left\lceil Q_{3}+C\right\rceil+\Gamma_{(1)}\right|
$$

where $Q_{3}$ is certain nef and big $\mathbb{Q}$-divisor on $F$. Applying the vanishing once more, we have

$$
\left|K_{F}+\left\lceil Q_{3}\right\rceil+\Gamma_{(1)}+C \|_{C}=\right| K_{C}+D \mid
$$

with $\operatorname{deg}(D)>\left(\Gamma_{(1)} \cdot C\right) \geq 2$. Thus $\Phi_{10}$ is birational by Lemma 7.1 and Claim [7.3.1.

When $\operatorname{dim} \operatorname{im}\left(\mu_{5}^{\prime}\right) \leq 2$, we have $h^{0}\left(X^{\prime}, F_{(1)}-2 F\right) \geq 1$ and hence $S_{5}-3 F \geq 0$. Therefore $\Phi_{10}$ is birational by Claim $7.7,2$ (5).

Part IV. $P_{6} \geq 14$. We set $m_{1}=6$. By Claim 7.7.1 and Claim 7.7.2 (1), (2) and (4), we may assume $2 \leq\left(T_{6} \cdot C\right) \leq 3$ and $h^{0}\left(F, T_{6}\right) \geq 4$. Clearly $\left|T_{6}\right|$ is not composed of the same pencil as that of $|C|$. Thus, by the Riemann-Roch theorem and the Clifford theorem, $\operatorname{dim} \operatorname{im}\left(\nu_{6}\right)=$ $h^{0}\left(C,\left.T_{6}\right|_{C}\right)=2$.

$(\mathbf{I V}-\mathbf{1})$. If $h^{0}\left(F, T_{6}\right) \geq 5$, then we see $h^{0}\left(F, T_{6}-C\right) \geq 3$. We pick a general member $C_{(1)}$ in Mov $\left|T_{6}-C\right|$. By Claim 7.7.2 (5), we may assume that $\left|C_{(1)}\right|$ is not composed of the same pencil as that of $|C|$. We shall analyze the natural map $\nu_{6}^{\prime}: H^{0}\left(F, C_{(1)}\right) \mapsto H^{0}\left(C,\left.C_{(1)}\right|_{C}\right)$. Clearly $2 \leq \operatorname{dimim}\left(\nu_{6}^{\prime}\right) \leq h^{0}\left(C,\left.T_{6}\right|_{C}\right)=2$.

Since $C_{(1)}$ is not algebraically equivalent to $C$, one has $\left(C_{(1)} \cdot C\right) \geq 2$. By the vanishing theorem, we have

$$
\begin{aligned}
\mid 10 K_{X^{\prime}} \|_{F} & \succeq \mid K_{X^{\prime}}+\left\lceil\pi^{*}\left(K_{X}\right)\right\rceil+S_{6}+F \|_{F} \\
& \succeq\left|K_{F}+\left\lceil\left.\pi^{*}\left(K_{X}\right)\right|_{F}\right\rceil+C_{(1)}+C\right|
\end{aligned}
$$

and $\left|K_{F}+\left\lceil\left.\pi^{*}\left(K_{X}\right)\right|_{F}\right\rceil+C_{(1)}+C \|_{C}=\right| K_{C}+D \mid$ with $\operatorname{deg}(D)>$ $\left(C_{(1)} \cdot C\right)=2$. Thus $\Phi_{10}$ is birational by Lemma 7.1 and Claim 7.3.1. 
(IV-2). If $h^{0}\left(F, T_{6}\right)=4$, we have $h^{0}\left(S_{6}-F\right) \geq 10$. We pick a general member $F_{(1)} \in \operatorname{Mov}\left|S_{6}-F\right|$ and consider the natural map:

$$
H^{0}\left(X^{\prime}, F_{(1)}\right) \stackrel{\mu_{6}^{\prime}}{\longrightarrow} H^{0}\left(F,\left.F_{(1)}\right|_{F}\right) \subset H^{0}\left(F,\left.S_{6}\right|_{F}\right) .
$$

Clearly we have $\operatorname{dimim}\left(\mu_{6}^{\prime}\right) \leq h^{0}\left(F, T_{6}\right)=4$.

When $\operatorname{dim} \operatorname{im}\left(\mu_{6}^{\prime}\right) \leq 3$, we have $F_{(1)}-3 F \geq 0$ and then $S_{6} \geq 4 F$. By Claim 7.7,2 (5), $\Phi_{10}$ is birational.

When $\operatorname{dim} \operatorname{im}\left(\mu_{6}^{\prime}\right)=4$, we see $\operatorname{dim} \nu_{6}\left(\operatorname{im}\left(\mu_{6}^{\prime}\right)\right)=\operatorname{dim} \nu_{6}\left(\operatorname{im}\left(\mu_{6}\right)\right)=2$. Thus $h^{0}\left(F,\left.F_{(1)}\right|_{F}-C\right)=2$. Furthermore $\left|F_{(1)}\right|_{F} \mid$ is not composed of the same pencil as that of $|C|$. Noting that a divisor of degree 1 can not move on $C$, we see $\left(F_{(1)} \cdot C\right) \geq 2$. Denote by $\Gamma_{(1)}$ a general irreducible element of $\mathrm{Mov}\left|F_{(1)}\right| F_{F}-C \mid$. Noting that $S_{6} \geq F_{(1)}+F$ and applying the vanishing theorem, we have

$$
\begin{aligned}
\left|10 K_{X^{\prime}}\right| & \succeq\left|K_{X^{\prime}}+\left\lceil 3 \pi^{*}\left(K_{X}\right)\right\rceil+F_{(1)}+F\right| \\
& \succeq\left|K_{F}+\left\lceil\left. 3 \pi^{*}\left(K_{X}\right)\right|_{F}\right\rceil+F_{(1)}\right|{ }_{F} \mid .
\end{aligned}
$$

If $\Gamma_{(1)}$ is not algebraically equivalent to $C$, we have $\left(\Gamma_{(1)} \cdot C\right) \geq 2$. The vanishing theorem gives

$$
\left|K_{F}+\left\lceil\left. 3 \pi^{*}\left(K_{X}\right)\right|_{F}\right\rceil+\Gamma_{(1)}+C \|_{C}=\right| K_{C}+D \mid
$$

with $\operatorname{deg}(D)>\left(\Gamma_{(1)} \cdot C\right) \geq 2$. Thus $\Phi_{10}$ is birational by Lemma 7.1 and Claim 7.3.1. If $\Gamma_{(1)}$ is algebraically equivalent to $C$, we have $\left.F_{(1)}\right|_{F} \geq 2 C$ and write

$$
\left.F_{(1)}\right|_{F}=2 C+H_{6}
$$

where $H_{6}$ is an effective divisor on $F$. Since $\left.3 \pi^{*}\left(K_{X}\right)\right|_{F}+\left.F_{(1)}\right|_{F}-C-$ $\frac{1}{2} H_{6}$ is nef and big, the Kawamata-Viehweg vanishing theorem implies the following surjective map

$$
H^{0}\left(F, K_{F}+\left\lceil\left. 3 \pi^{*}\left(K_{X}\right)\right|_{F}+\left.F_{(1)}\right|_{F}-\frac{1}{2} H_{6}\right\rceil\right) \longrightarrow H^{0}\left(C, D^{\prime}\right)
$$

where $D^{\prime}:=\left.\left\lceil\left. 3 \pi^{*}\left(K_{X}\right)\right|_{F}+\left.F_{(1)}\right|_{F}-\frac{1}{2} H_{6}-C\right\rceil\right|_{C}$ with $\operatorname{deg}\left(D^{\prime}\right) \geq 3 \xi+$ $\frac{1}{2}\left(F_{(1)} \cdot C\right)>2$. Thus we see that $\Phi_{10}$ is birational again by Lemma 7.1 and Claim 7.3.1. So we conclude the theorem.

Corollary 7.8. (=Theorem 1.8(2)) Let $X$ be a minimal projective 3fold of general type with $\delta(X)=2$. If $\Phi_{10}$ is not birational, then the weighted basket $\mathbb{B}(X)=\left(B_{X}, P_{2}, \chi\left(\mathcal{O}_{X}\right)\right)$ are dominated by an initial basket listed in Tables II-1, II-2, II-3 in Appendix.

Proof. By Lemma 7.6 and Theorem [7.7, we see $0 \leq \chi\left(\mathcal{O}_{X}\right) \leq 3$, $P_{2}(X)=2, P_{3}(X) \leq 3, P_{4}(X) \leq 5, P_{5}(X) \leq 7$ and $P_{6}(X) \leq 13$. According to [6, Section 3], the total number of numerical types of $\mathbb{B}(X)$ is finite. We give a list of $\mathbb{B}^{0}(X)$ in Tables II-1, II-2 and II-3. 


\section{Projective 4-folds of general type with positive geometric genus}

In order to study 4 -folds of general type, we need to prove a slightly general statement on 3 -folds.

Theorem 8.1. Let $\nu: \tilde{X} \longrightarrow X$ be a birational morphism from a nonsingular projective 3-fold $\tilde{X}$ of general type onto a minimal model $X$ with $p_{g}(X)>0$. Let $Q_{\lambda}$ be any $\mathbb{Q}$-divisor on $\tilde{X}$ satisfying $Q_{\lambda} \equiv$ $\lambda \nu^{*}\left(K_{X}\right)$ for some rational number $\lambda>16$. Then $\left|K_{\tilde{X}}+\left\lceil Q_{\lambda}\right\rceil\right|$ gives a birational map onto its image. In particular, $\Phi_{m}$ is birational for all $m \geq 18$.

Proof. From the proof of Corollary 4.10, we only need to consider the following two cases:

Case 1. $P_{4} \geq 2$;

Case 2. $P_{4}=1$ and $P_{5} \geq 3$.

Set $m_{0}=4$ (resp. 5) in Case 1 (resp. Case 2). Take a sub-pencil $\Lambda \subset\left|m_{0} K_{X}\right|$. We use the same set up as in 2.1. We may and do assume that $\pi$ factors through $\nu$, i.e. there is a birational morphism $\mu: X^{\prime} \longrightarrow \tilde{X}$ so that $\pi=\nu \circ \mu$ and that $\mu^{*}\left(\left\{Q_{\lambda}\right\}\right) \cup\{$ exc. divisors of $\mu\}$ has simple normal crossing supports.

Since

$$
\mu_{*} \mathcal{O}_{X^{\prime}}\left(K_{X^{\prime}}+\left\lceil\mu^{*}\left(Q_{\lambda}\right)\right\rceil\right) \subseteq \mu_{*} \mathcal{O}_{X^{\prime}}\left(K_{X^{\prime}}+\mu^{*}\left\lceil Q_{\lambda}\right\rceil\right) \subseteq \mathcal{O}_{\tilde{X}}\left(K_{\tilde{X}}+\left\lceil Q_{\lambda}\right\rceil\right),
$$

it is sufficient to prove the birationality of $\Phi_{\left|K_{X^{\prime}}+\left\lceil\mu^{*}\left(Q_{\lambda}\right)\right]\right|}$. We write $Q_{\lambda}^{\prime}:=\mu^{*}\left(Q_{\lambda}\right) \equiv \lambda \pi^{*}\left(K_{X}\right)$.

We have an induced fibration $f: X^{\prime} \longrightarrow \Gamma$ onto a smooth projective curve. Let $F$ be a general fiber of $f$. Recall that we have $m_{0} \pi^{*}\left(K_{X}\right) \sim_{\mathbb{Q}}$ $\theta F+E_{\Lambda}^{\prime}$ for a positive integer $\theta$ and an effective $\mathbb{Q}$-divisor $E_{\Lambda}^{\prime}$ on $X^{\prime}$.

Without lose of generality, we may assume $p_{g}(X)=1$ (the case with $p_{g}(X)>1$ is much easier). Clearly one has $p_{g}(F)>0$.

Claim 8.1.1. One has $h^{0}\left(X^{\prime}, K_{X^{\prime}}+\left\lceil Q_{\lambda}^{\prime}\right\rceil\right)>0$ for $\lambda>2 m_{0}+1$. By Lemma 2.1.

$$
\left.\pi^{*}\left(K_{X}\right)\right|_{F} \equiv \frac{1}{m_{0}+1} \sigma^{*}\left(K_{F_{0}}\right)+H_{m_{0}}
$$

for certain effective $\mathbb{Q}$-divisor $H_{m_{0}}$ on $F$. Since $Q_{\lambda}^{\prime}-F-\frac{1}{\theta} E_{\Lambda}^{\prime} \equiv$ $\left(\lambda-\frac{m_{0}}{\theta}\right) \pi^{*}\left(K_{X}\right)$ is nef and big, Kawamata-Viehweg vanishing implies the surjective map:

$$
H^{0}\left(X^{\prime}, K_{X^{\prime}}+\left\lceil Q_{\lambda}^{\prime}-\frac{1}{\theta} E_{\Lambda}^{\prime}\right\rceil\right) \longrightarrow H^{0}\left(F, K_{F}+\left.\left\lceil Q_{\lambda}^{\prime}-\frac{1}{\theta} E_{\Lambda}^{\prime}\right\rceil\right|_{F}\right) .
$$

Let

$$
\begin{aligned}
Q_{\lambda, F}: & =\left.\left(Q_{\lambda}^{\prime}-\frac{1}{\theta} E_{\Lambda}^{\prime}\right)\right|_{F}-\left(m_{0}+1\right) H_{m_{0}}-\sigma^{*}\left(K_{F_{0}}\right) \\
& \left.\equiv\left(\lambda-\frac{m_{0}}{\theta}-m_{0}-1\right) \pi^{*}\left(K_{X}\right)\right|_{F},
\end{aligned}
$$


which is nef and big. Since $p_{g}(F)>0$, we have

$$
\begin{aligned}
& h^{0}\left(F, K_{F}+\left.\left\lceil Q_{\lambda}^{\prime}-\frac{1}{\theta} E_{\Lambda}^{\prime}\right\rceil\right|_{F}\right) \\
\geq & h^{0}\left(F, K_{F}+\left\lceil\left.\left(Q_{\lambda}^{\prime}-\frac{1}{\theta} E_{\Lambda}^{\prime}\right)\right|_{F}-\left(m_{0}+1\right) H_{m_{0}}\right)\right\rceil \\
= & h^{0}\left(F, K_{F}+\left\lceil Q_{\lambda, F}\right\rceil+\sigma^{*}\left(K_{F_{0}}\right)\right) \geq 2
\end{aligned}
$$

by [7, Lemma 2.14]. This verifies the Claim.

Claim 8.1.2. The linear system $\left|K_{X^{\prime}}+\left\lceil Q_{\lambda}^{\prime}\right\rceil\right|$ distinguishes different general fibers of $f$ for any $\lambda>3 m_{0}+1$.

Proof. When $g(\Gamma)=0$, we consider $Q_{\zeta}^{\prime}:=Q_{\lambda}^{\prime}-F-\frac{1}{\theta} E_{\Lambda}^{\prime} \equiv \zeta \pi^{*}\left(K_{X}\right)$ with $\zeta=\lambda-\frac{m_{0}}{\theta}$. It is clear that $K_{X^{\prime}}+\left\lceil Q_{\lambda}^{\prime}\right\rceil \geq\left(K_{X^{\prime}}+\left\lceil Q_{\zeta}^{\prime}\right\rceil\right)+F$ and hence $\left|K_{X^{\prime}}+\left\lceil Q_{\lambda}^{\prime}\right\rceil\right|$ distinguishes different general fibers by Claim 8.1.1 since $\zeta>2 m_{0}+1$.

When $g(\Gamma)>0$, we have $\theta \geq 2$. Pick two different general fibers $F_{1}$ and $F_{2}$ of $f$. The vanishing theorem gives the surjective map:

$$
\begin{aligned}
& H^{0}\left(X^{\prime}, K_{X^{\prime}}+\left\lceil Q_{\lambda}^{\prime}-\frac{2}{\theta} E_{\Lambda}^{\prime}\right\rceil\right) \\
\longrightarrow & \oplus_{i=1}^{2} H^{0}\left(F_{i},\left.\left(K_{X^{\prime}}+\left\lceil Q_{\lambda}^{\prime}-F_{1}-F_{2}-\frac{2}{\theta} E_{\Lambda}^{\prime}\right\rceil+F_{1}+F_{2}\right)\right|_{F_{i}}\right)
\end{aligned}
$$

where we note that $\left.\left(K_{X^{\prime}}+\left\lceil Q_{\lambda}^{\prime}-F_{1}-F_{2}-\frac{2}{\theta} E_{\Lambda}^{\prime}\right\rceil\right)\right|_{F_{i}} \geq 0$ due to Claim 8.1.1 and the fact $\left.\left(F_{1}+F_{2}\right)\right|_{F_{i}}=0$. Hence $\left|K_{X^{\prime}}+\left\lceil Q_{\lambda}^{\prime}\right\rceil\right|$ distinguishes $F_{1}$ and $F_{2}$.

Now we discuss two cases independently.

Case 1. $P_{4} \geq 2$.

If $F$ is a $(1,2)$ surface, we take $|G|:=\operatorname{Mov}\left|\sigma^{*}\left(K_{F_{0}}\right)\right|$ and a general member $C \in|G|$. By the surjection map in (8.1) and Claim 8.1.2, it is sufficient to study the linear system $\left|K_{F}+\left\lceil\left.\left(Q_{\lambda}^{\prime}-\frac{1}{\theta} E_{\Lambda}^{\prime}\right)\right|_{F}\right\rceil\right|$. For any $t$, let

$$
L_{\lambda, t}:=\left.\left(Q_{\lambda}^{\prime}-\frac{1}{\theta} E_{\Lambda}^{\prime}\right)\right|_{F}-t \sigma^{*}\left(K_{F_{0}}\right)-\left.5 t H_{4} \equiv\left(\lambda-\frac{4}{\theta}-5 t\right) \pi^{*}\left(K_{X}\right)\right|_{F},
$$

which is nef and big as long as $\lambda-\frac{4}{\theta}-5 t>0$. Notice also that $\left.\left(Q_{\lambda}^{\prime}-\frac{1}{\theta} E_{\Lambda}^{\prime}\right)\right|_{F} \geq L_{\lambda, t}+t \sigma^{*}\left(K_{F_{0}}\right)$. For simplicity, $L_{\lambda, 0}$ is denoted by $L_{\lambda}$. In fact, for $\lambda>14$ and by [7, Lemma 2.14], one has

$$
K_{F}+\left.\left\lceil Q_{\lambda}^{\prime}-\frac{1}{\theta} E_{\Lambda}^{\prime}\right\rceil\right|_{F} \geq\left(K_{F}+\left\lceil L_{\lambda, 2}\right\rceil+\sigma^{*}\left(K_{F_{0}}\right)\right)+C \geq C .
$$

Thus $\left|K_{F}+\left\lceil\left.\left(Q_{\lambda}^{\prime}-\frac{1}{\theta} E_{\Lambda}^{\prime}\right)\right|_{F}\right\rceil\right|$ separates different general curves $C$ when $\lambda>14$. Restricting to the curve $C$, one sees by the vanishing theorem that

$$
\left|K_{F}+\left\lceil\left.\left(Q_{\lambda}^{\prime}-\frac{1}{\theta} E_{\Lambda}^{\prime}\right)\right|_{F}\right\rceil_{\mid C} \geq\right| K_{F}+\left\lceil L_{\lambda, 1}\right\rceil+\left.C\right|_{\mid C}=\left|K_{C}+\left\lceil L_{\lambda, 1}\right\rceil\right|_{C} \mid .
$$


Since $\operatorname{deg}\left(\left.\left\lceil L_{\lambda, 1}\right\rceil\right|_{C}\right) \geq\left(\lambda-\frac{4}{\theta}-5\right) \xi>2$ for $\xi \geq 2 / 7$ (cf. Table A3 with $\left.m_{0}=4\right)$. Thus $\Phi_{\left|K_{X^{\prime}}+\left\lceil Q_{\lambda}^{\prime}\right\rceil\right|}$ separates points on the general curve $C$ and hence is birational when $\lambda>16$.

Assume that $F$ is not a $(1,2)$ surface. We would like to study $\mid K_{F}+$ $\left\lceil L_{\lambda}\right\rceil \mid$ where $L_{\lambda}:=\left.\left(Q_{\lambda}^{\prime}-\frac{1}{\theta} E_{\Lambda}^{\prime}\right)\right|_{F}$, making use of the relation (8.1). If $K_{F_{0}}^{2} \geq 2$, Inequalities (3.4), (3.6) imply

$$
L_{\lambda}^{2} \geq \frac{2(\lambda-4)^{2}}{25}>8
$$

whenever $\lambda>14$. If $F$ is a $(1,1)$ surface, then we have $q(X)=g(\Gamma) \geq 0$ and $h^{2}\left(\mathcal{O}_{X}\right)=0$ as seen in the proof of Case 2 of Corollary 4.10. Hence we have $\chi\left(\mathcal{O}_{X}\right) \leq 0$ and Reid's Riemann-Roch formula gives $P_{5}>P_{4} \geq 2$. In particular, we have $P_{5} \geq 3$. We omit the discussion for the situation when $\left|5 K_{X^{\prime}}\right|$ and $\left|4 K_{X^{\prime}}\right|$ are composed with the same pencil since that is a comparatively much better case. So may assume that $\left.\left|5 K_{X^{\prime}}\right|\right|_{F}$ is moving on $F$. If we take $\left|G_{1}\right|:=\operatorname{Mov} \mid\left\lceil\left. 5 \pi^{*}\left(K_{X}\right)\right|_{F} \mid\right.$, we have $\beta_{G_{1}}=\frac{1}{5}$. Then, by Lemma 2.1 and Lemma 2.4, we have

$$
L_{\lambda}^{2} \geq \frac{(\lambda-4)^{2}}{25}\left(\sigma^{*}\left(K_{F_{0}}\right) \cdot G_{1}\right) \geq \frac{2(\lambda-4)^{2}}{25}>8
$$

whenever $\lambda>14$. Finally, for both cases, $\left(L_{\lambda} \cdot \tilde{C}\right) \geq \frac{2(\lambda-4)}{5} \geq 4$ for $\lambda \geq 14$ and for any very general curve $\tilde{C}$ on $F$. Therefore, by Lemma 2.3. $\left|K_{F}+\left\lceil L_{\lambda}\right\rceil\right|$ gives a birational map when $\lambda \geq 14$.

Hence, when $P_{4} \geq 2, \Phi_{\left|K_{X^{\prime}}+\left\lceil Q_{\lambda}^{\prime}\right\rceil\right|}$ is birational for $\lambda>16$.

Case 2. $P_{4}=1$ and $P_{5} \geq 3$.

We set $m_{0}=5$. If $d_{5}=1$, we set $\Lambda=\left|5 K_{X}\right|$. Then we are in much better situation than that of $P_{3}=2$ since we have $\theta \geq 2$ (and noting that $\frac{\theta}{m_{0}}=\frac{2}{5}>\frac{1}{3}$ ). We omit the details and leave this as an exercise to interested readers.

If $d_{5} \geq 2$, we take a sub-pencil $\Lambda \subset\left|5 K_{X}\right|$ and $\Lambda$ induces a fibration $f: X^{\prime} \longrightarrow \Gamma$ onto a smooth complete curve $\Gamma$. As we have seen in Case 3 of Corollary 4.10 , the general fiber $F$ satisfies $K_{F_{0}}^{2} \geq 2$. For the similar reason, we can take $m_{1}=5$ and $|G|:=\operatorname{Mov}\left|m_{1} K_{X^{\prime}}\right|_{F} \mid$. Pick a generic irreducible element $C$ in $|G|$. Lemma 2.1 implies $\xi=$ $\left(\pi^{*}\left(K_{X}\right) \cdot C\right) \geq \frac{1}{6}\left(\sigma^{*}\left(K_{F_{0}}\right) \cdot C\right) \geq \frac{1}{3}$. We may write $\left.5 \pi^{*}\left(K_{X}\right)\right|_{F} \equiv C+N_{5}$ for an effective $\mathbb{Q}$-divisor $N_{5}$ on $F$. For two different generic irreducible curves $C_{1}$ and $C_{2}$ in $|G|$, we set

$$
L_{\lambda, 2}:=\left.\left(Q_{\lambda}^{\prime}-\frac{1}{\theta} E_{\Lambda}^{\prime}\right)\right|_{F}-C_{1}-C_{2}-2 N_{5},
$$

and

$$
L_{\lambda, 1}:=\left.\left(Q_{\lambda}^{\prime}-\frac{1}{\theta} E_{\Lambda}^{\prime}\right)\right|_{F}-C-N_{5}
$$

respectively. It is clear that they are both nef and big whenever $\lambda>15$. 
Thanks to the vanishing theorem, we have the surjective map:

$$
\begin{aligned}
H^{0}\left(F, K_{F}+\left\lceil L_{\lambda}-2 N_{5}\right\rceil\right) \longrightarrow & H^{0}\left(C_{1}, K_{C_{1}}+\left.\left\lceil L_{\lambda, 2}\right\rceil\right|_{C_{1}}+\left.C_{2}\right|_{C_{1}}\right) \\
& \oplus H^{0}\left(C_{2}, K_{C_{2}}+\left.\left\lceil L_{\lambda, 2}\right\rceil\right|_{C_{2}}+\left.C_{1}\right|_{C_{2}}\right)
\end{aligned}
$$

if $\lambda>15$. It is clear that

$$
H^{0}\left(C_{i}, K_{C_{i}}+\left\lceil L_{\lambda, 2}\right\rceil_{\mid C_{i}}+\left.C_{2-i}\right|_{C_{i}}\right) \neq 0
$$

since $L_{\lambda, 2}$ is nef and big. Hence $\left|K_{F}+\left\lceil\left.\left(Q_{\lambda}^{\prime}-\frac{1}{\theta} E_{\Lambda}^{\prime}\right)\right|_{F}-2 N_{5}\right\rceil\right|=$ $\left|K_{F}+\left\lceil L_{\lambda}-2 N_{5}\right\rceil\right|$ separates different general curves $C$ in $|G|$. This also implies that $\left|K_{F}+\left\lceil\left(Q_{\lambda}^{\prime}-\frac{1}{\theta} E_{\Lambda}^{\prime}\right)\right\rceil\right|$ can distinguishes $C_{1}$ and $C_{2}$. Now applying the vanishing theorem once more, we get the surjective map:

$$
H^{0}\left(F, K_{F}+\left\lceil L_{\lambda}-N_{5}\right\rceil\right) \longrightarrow H^{0}\left(C, K_{C}+\left\lceil L_{\lambda, 1}\right\rceil_{\mid C}\right)
$$

with

$$
\operatorname{deg}\left(\left\lceil L_{\lambda, 1}\right\rceil_{\mid C}\right) \geq\left(\lambda-\frac{5}{\theta}-5\right) \xi>2
$$

whenever $\lambda>16$ for $\xi \geq 1 / 3$. Thus, by Theorem $2.7,\left|K_{X^{\prime}}+\left\lceil Q_{\lambda}^{\prime}\right\rceil\right|$ gives a birational map for $\lambda>16$. So we conclude the statement of the theorem.

Theorem 8.2. (=Theorem 1.11) Let $V$ be a nonsingular projective 4-fold of general type. Then,

(1) when $p_{g}(V) \geq 2, \Phi_{m, V}$ is birational for all $m \geq 35$;

(2) when $p_{g}(V) \geq 19, \Phi_{m, V}$ is birational for all $m \geq 18$.

Proof. Let $Z$ be the minimal model of $V$. We set $m_{0}=1, \Lambda=\left|K_{Z}\right|$ and use the set up in 2.1. Thus we have an induced fibration $f: Z^{\prime} \longrightarrow \Gamma$.

First we consider the case $\operatorname{dim} \Gamma=1$. Recall that we have $M_{\Lambda} \equiv \theta F$ for a general fiber $F$ of $f$, where $\theta \geq p_{g}(Z)-1$. It is clear that, when $m \geq 3,\left|m K_{Z^{\prime}}\right|$ distinguishes different general fibers of $f$. Pick a general fiber $F=X^{\prime}$, which is a nonsingular projective 3-fold of general type with $p_{g}\left(X^{\prime}\right)>0$. Replace by its birational model, we may assume that there is a birational morphism $\nu: X^{\prime} \longrightarrow X$ onto a minimal model. By Lemma 2.1, we have

$$
\left.\pi^{*}\left(K_{Z}\right)\right|_{X^{\prime}} \equiv \frac{\theta}{\theta+1} \nu^{*}\left(K_{X}\right)+J_{1}
$$

for an effective $\mathbb{Q}$-divisor $J_{1}$ on $X^{\prime}$. When $m$ is large, since $(m-$ 1) $\pi^{*}\left(K_{Z}\right)-X^{\prime}-\frac{1}{\theta} E_{\Lambda}^{\prime}$ is nef and big, Kawamata-Viehweg vanishing implies:

$$
\begin{aligned}
& \left.\left|K_{Z^{\prime}}+\left\lceil(m-1) \pi^{*}\left(K_{Z}\right)-\frac{1}{\theta} E_{\Lambda}^{\prime}\right\rceil\right|\right|_{X^{\prime}} \\
= & \left|K_{X^{\prime}}+\left\lceil(m-1) \pi^{*}\left(K_{Z}\right)-\frac{1}{\theta} E_{\Lambda}^{\prime}\right\rceil X_{X^{\prime}}\right| \\
\succeq & \left|K_{X^{\prime}}+\left\lceil R_{m}\right\rceil\right|
\end{aligned}
$$


where $R_{m}:=\left.\left((m-1) \pi^{*}\left(K_{Z}\right)-X^{\prime}-\frac{1}{\theta} E_{\Lambda}^{\prime}\right)\right|_{X^{\prime}}$. In fact, we have

$$
\begin{aligned}
R_{m} & \left.\equiv\left(m-1-\frac{1}{\theta}\right) \pi^{*}\left(K_{Z}\right)\right|_{X^{\prime}} \\
& \equiv\left(\frac{m \theta}{\theta+1}-1\right) \nu^{*}\left(K_{X}\right)+\left(m-1-\frac{1}{\theta}\right) J_{1}
\end{aligned}
$$

Since $\frac{m \theta}{\theta+1}-1>16$ whenever either $m \geq 18$ and $p_{g}(Z) \geq 19$ or $m \geq 35$ and $p_{g}(Z) \geq 2$, Theorem 8.1 implies that $\left|K_{X^{\prime}}+\left\lceil R_{m}-\left(m-1-\frac{1}{p}\right) J_{1}\right\rceil\right|$ gives a birational map. Thus statements of the theorem follow in this case.

Next we consider the case $\operatorname{dim} \Gamma \geq 2$. By definition, $\theta=1$. Clearly it is sufficient to consider $\left.\Phi_{\left|m K_{Z^{\prime}}\right|}\right|_{X^{\prime}}$ for a general member $X^{\prime} \in\left|M_{\Lambda}\right|$. We consider a general $X^{\prime}$ and, similarly, we may assume that there is a birational morphism $\nu: X^{\prime} \longrightarrow X$ onto a minimal model $X$. Then Kawamata's extension theorem [20, Theorem A] still implies

$$
\left.\pi^{*}\left(K_{Z}\right)\right|_{X^{\prime}} \geq \frac{1}{2} \nu^{*}\left(K_{X}\right) .
$$

We consider the linear system $\left|M_{\Lambda}\right|_{X^{\prime}} \mid$, which may be assumed to be base point free modulo further birational modifications. Pick a generic irreducible element $S$ of this linear system. We clearly have

$$
\left.\pi^{*}\left(K_{Z}\right)\right|_{X^{\prime}} \geq\left. M_{\Lambda}\right|_{X^{\prime}} \geq S
$$

Modulo $\mathbb{Q}$-linear equivalence, one has

$$
2 S \leq\left.\left(\pi^{*}\left(K_{Z}\right)+X^{\prime}\right)\right|_{X^{\prime}} \leq K_{X^{\prime}}
$$

Thus Kawamata's extension theorem gives

$$
\left.\nu^{*}\left(K_{X}\right)\right|_{S} \geq \frac{2}{3} \sigma^{*}\left(K_{S_{0}}\right)
$$

where $\sigma: S \longrightarrow S_{0}$ is the contraction onto the minimal model $S_{0}$ of $S$. Both (8.2) and (8.3) imply

$$
\left.\pi^{*}\left(K_{Z}\right)\right|_{S} \geq \frac{1}{3} \sigma^{*}\left(K_{S_{0}}\right) .
$$

Write $\left.\pi^{*}\left(K_{Z}\right)\right|_{X^{\prime}} \equiv S+H_{\Lambda}$ where $H_{\Lambda}$ is an effective $\mathbb{Q}$-divisor on $X^{\prime}$. Since $R_{m}-S-\left.H_{\Lambda} \equiv(m-3) \pi^{*}\left(K_{Z}\right)\right|_{X^{\prime}}$ is nef and big, the vanishing theorem implies

$$
\begin{aligned}
\left|K_{X^{\prime}}+\left\lceil R_{m}-H_{\Lambda}\right\rceil\right|_{\mid S} & =\left|K_{S}+\left\lceil R_{m}-S-H_{\Lambda}\right\rceil_{\mid S}\right| \\
& \succeq\left|K_{S}+\left\lceil R_{m, S}\right\rceil\right|
\end{aligned}
$$

where $R_{m, S}:=\left.\left(R_{m}-S-H_{\Lambda}\right)\right|_{S}$. Note that

$$
\begin{aligned}
R_{m, S} & \left.\equiv(m-3) \pi^{*}\left(K_{Z}\right)\right|_{S} \\
& \equiv \frac{m-3}{3} \sigma^{*}\left(K_{S_{0}}\right)+E_{m, S}
\end{aligned}
$$

where $E_{m, S}$ is an effective $\mathbb{Q}$-divisor on $S$. Now it is clear by Lemma 2.3 that $\left|K_{S}+\left\lceil R_{m, S}-E_{m, S}\right\rceil\right|$ gives a birational map whenever $m \geq 15$. 
Again Kawamata-Viehweg vanishing shows that $\left|K_{X^{\prime}}+\left\lceil R_{m}\right\rceil\right|$ distinguishes different elements $S$. Thus we have shown that $\Phi_{m, Z}$ is birational for all $m \geq 15$ in this case. We are done.

G. Brown and M. Reid kindly informed us the following interesting canonical 4-folds:

Example 8.3. The general hypersurfaces $W_{36} \subset \mathbb{P}(1,1,3,5,7,18)$ and $Y_{36} \subset \mathbb{P}(1,1,4,5,6,18)$ have canonical singularities, $p_{g}=2$. It is clear that the 17 -canonical maps of these two 4 -folds are not birational.

Problem 8.4. It is a very interesting problem to find more examples of 4 -folds of general type so that $\Phi_{m}$ is not birational for large $m$.

\section{Appendix. Tables}

Table F-0
\begin{tabular}{|l|l|l|l|l|}
\hline Types & $B_{X}$ & $\chi$ & $K_{X}^{3}$ & $\delta(X)$ \\
\hline $2 \mathrm{a}$ & $\{4 \times(1,2),(4,9),(2,5),(5,13), 3 \times(1,3), 2 \times(1,4)\}$ & 2 & $1 / 1170$ & 18 \\
\hline 41 & $\{5 \times(1,2),(4,9), 2 \times(3,8),(1,3), 2 \times(2,7)\}$ & 2 & $1 / 252$ & 13 \\
\hline
\end{tabular}

Table F-1

\begin{tabular}{|l|l|l|l|l|}
\hline Types & $B_{X}$ & $\chi$ & $K_{X}^{3}$ & $\delta(X)$ \\
\hline 2 & $\{4 \times(1,2),(4,9), 2 \times(2,5),(3,8), 3 \times(1,3), 2 \times(1,4)\}$ & 2 & $1 / 360$ & 15 \\
\hline 3 & $\{6 \times(1,2),(5,11), 4 \times(2,5),(3,8), 4 \times(1,3),(2,7), 2 \times(1,4)\}$ & 3 & $23 / 9240$ & 15 \\
\hline 5.1 & $\{7 \times(1,2),(4,9), 3 \times(2,5),(5,13), 4 \times(1,3),(3,11),(1,4)\}$ & 3 & $61 / 25740$ & 15 \\
\hline 5.2 & $\{7 \times(1,2),(4,9), 2 \times(2,5),(7,18), 4 \times(1,3),(3,11),(1,4)\}$ & 3 & $1 / 660$ & 15 \\
\hline 5.3 & $\{7 \times(1,2),(4,9),(2,5),(9,23), 4 \times(1,3),(3,11),(1,4)\}$ & 3 & $47 / 45540$ & 15 \\
\hline $5 \mathrm{a}$ & $\{7 \times(1,2),(4,9),(11,28), 4 \times(1,3),(3,11),(1,4)\}$ & 3 & $1 / 1386$ & 15 \\
\hline 5b & $\{7 \times(1,2),(4,9), 3 \times(2,5),(5,13), 4 \times(1,3),(4,15)\}$ & 3 & $1 / 1170$ & 15 \\
\hline $53 \mathrm{a}$ & $\{3 \times(1,2),(4,9), 2 \times(2,5),(5,13), 3 \times(1,3),(1,5)\}$ & 2 & $1 / 1170$ & 15 \\
\hline
\end{tabular}

\section{Table F-2}

\begin{tabular}{|l|l|l|l|l|}
\hline Type & $B_{X}$ & $\chi$ & $K_{X}^{3}$ & $\delta(X)$ \\
\hline 1 & $\{5 \times(1,2),(3,7), 3 \times(2,5), 3 \times(1,3),(3,11)\}$ & 2 & $3 / 770$ & 14 \\
\hline 4 & $\{7 \times(1,2),(4,9), 4 \times(2,5),(4,11), 3 \times(1,3),(2,7), 2 \times(1,4)\}$ & 3 & $13 / 3465$ & 14 \\
\hline 4.5 & $\{7 \times(1,2),(4,9), 4 \times(2,5),(5,14), 2 \times(1,3),(2,7), 2 \times(1,4)\}$ & 3 & $1 / 630$ & 14 \\
\hline 5 & $\{7 \times(1,2),(4,9), 4 \times(2,5),(3,8), 4 \times(1,3),(3,11),(1,4)\}$ & 3 & $17 / 3960$ & 14 \\
\hline 5.4 & $\{7 \times(1,2),(4,9), 4 \times(2,5),(3,8), 4 \times(1,3),(4,15)\}$ & 3 & $1 / 360$ & 14 \\
\hline 6 & $\{9 \times(1,2), 2 \times(3,7),(2,5),(4,11), 4 \times(1,3), 2 \times(2,7),(1,5)\}$ & 3 & $1 / 462$ & 14 \\
\hline 7 & $\{5 \times(1,2),(4,9),(3,7), 5 \times(1,3),(2,7),(1,5)\}$ & 2 & $1 / 630$ & 14 \\
\hline $7 \mathrm{a}$ & $\{5 \times(1,2),(7,16), 5 \times(1,3),(2,7),(1,5)\}$ & 2 & $1 / 1680$ & 14 \\
\hline 10 & $\{8 \times(1,2),(4,9),(3,7), 2 \times(3,8), 5 \times(1,3),(2,7),(1,4),(1,5)\}$ & 3 & $1 / 630$ & 14 \\
\hline 11 & $\{9 \times(1,2), 2 \times(3,7),(3,8),(4,11), 3 \times(1,3),(3,10),(1,4),(1,5)\}$ & 3 & $3 / 3080$ & 14 \\
\hline 12 & $\{9 \times(1,2),(4,9),(2,5), 2 \times(3,8), 4 \times(1,3), 2 \times(2,7),(1,5)\}$ & 3 & $1 / 252$ & 14 \\
\hline 12.1 & $\{9 \times(1,2),(4,9),(5,13),(3,8), 4 \times(1,3), 2 \times(2,7),(1,5)\}$ & 3 & $67 / 32760$ & 14 \\
\hline $12 \mathrm{a}$ & $\{9 \times(1,2),(4,9),(8,21), 4 \times(1,3), 2 \times(2,7),(1,5)\}$ & 3 & $1 / 630$ & 14 \\
\hline 14 & $\begin{array}{l}\{10 \times(1,2),(3,7), 2 \times(2,5), 2 \times(3,8), 6 \times(1,3), 2 \times(2,7), \\
(1,4),(1,5)\}\end{array}$ & 4 & $1 / 770$ & 14 \\
\hline 15 & $\begin{array}{l}\{11 \times(1,2),(4,9),(3,7), 2 \times(2,5),(3,8),(4,11), 5 \times(1,3), 2 \times(2,7), \\
(1,4),(1,5)\}\end{array}$ & 4 & $71 / 27720$ & 14 \\
\hline 15.1 & $\begin{array}{l}\{11 \times(1,2),(4,9),(3,7), 2 \times(2,5),(7,19), 5 \times(1,3), 2 \times(2,7), \\
(1,4),(1,5)\}\end{array}$ & 4 & $47 / 23940$ & 14 \\
\hline 15.2 & $\{11 \times(1,2),(7,16), 2 \times(2,5),(3,8),(4,11), 5 \times(1,3)$, & & \\
& $2 \times(2,7),(1,4),(1,5)\}$ & 4 & $29 / 18480$ & 14 \\
\hline 16 & $\{11 \times(1,2),(4,9),(3,7), 2 \times(2,5), 2 \times(3,8), 6 \times(1,3),(2,7)$, & 4 & $43 / 13860$ & 14 \\
\hline
\end{tabular}




\begin{tabular}{|c|c|c|c|c|}
\hline 16.1 & $\begin{array}{l}\{11 \times(1,2),(4,9),(3,7),(2,5),(5,13),(3,8), 6 \times(1,3),(2,7), \\
(3,11),(1,5)\}\end{array}$ & 4 & $85 / 72072$ & 14 \\
\hline 16.2 & $\begin{array}{l}\{11 \times(1,2),(7,16), 2 \times(2,5), 2 \times(3,8), 6 \times(1,3),(2,7), \\
(3,11),(1,5)\}\end{array}$ & 4 & $13 / 6160$ & 14 \\
\hline 16.4 & $\{11 \times(1,2),(7,16), 2 \times(2,5), 2 \times(3,8), 6 \times(1,3),(5,18),(1,5)\}$ & 4 & $1 / 720$ & 14 \\
\hline 16.5 & $\begin{array}{l}\{11 \times(1,2),(4,9),(3,7), 2 \times(2,5), 2 \times(3,8), 6 \times(1,3),(5,18), \\
(1,5)\}\end{array}$ & 4 & $1 / 420$ & 14 \\
\hline 17 & $\{9 \times(1,2), 2 \times(3,7), 2 \times(4,11), 3 \times(1,3),(2,7),(1,4),(1,5)\}$ & 3 & $3 / 1540$ & 14 \\
\hline 18 & $\{9 \times(1,2), 2 \times(3,7),(3,8),(4,11), 4 \times(1,3),(3,11),(1,5)\}$ & 3 & $23 / 9240$ & 14 \\
\hline $18 \mathrm{~b}$ & $\{9 \times(1,2), 2 \times(3,7),(7,19), 4 \times(1,3),(3,11),(1,5)\}$ & 3 & $83 / 43890$ & 14 \\
\hline 20 & $\{7 \times(1,2), 2 \times(4,9),(2,5),(3,8), 6 \times(1,3),(2,7),(1,4),(1,5)\}$ & 3 & $1 / 504$ & 14 \\
\hline 21 & $\{6 \times(1,2),(4,9),(3,8), 3 \times(1,3),(3,10),(1,5)\}$ & 2 & $1 / 360$ & 14 \\
\hline 23 & $\begin{array}{l}\{8 \times(1,2),(4,9),(3,7),(2,5),(4,11), 4 \times(1,3),(3,10),(1,4), \\
(1,5)\}\end{array}$ & 3 & $19 / 13860$ & 14 \\
\hline 25 & $\begin{array}{l}\{9 \times(1,2),(5,11),(4,9), 3 \times(2,5),(3,8), 7 \times(1,3), 2 \times(2,7), \\
(1,4),(1,5)\}\end{array}$ & 4 & $47 / 27720$ & 14 \\
\hline $25 \mathrm{a}$ & $\begin{array}{l}\{9 \times(1,2),(9,20), 3 \times(2,5),(3,8), 7 \times(1,3), 2 \times(2,7),(1,4), \\
(1,5)\}\end{array}$ & 4 & $1 / 840$ & 14 \\
\hline 26 & $\begin{array}{l}\{10 \times(1,2), 2 \times(4,9), 3 \times(2,5),(4,11), 6 \times(1,3), 2 \times(2,7), \\
(1,4),(1,5)\}\end{array}$ & 4 & $41 / 13860$ & 14 \\
\hline 27 & $\begin{array}{l}\{10 \times(1,2), 2 \times(4,9), 3 \times(2,5),(3,8), 7 \times(1,3),(2,7) \\
(3,11),(1,5)\}\end{array}$ & 4 & $97 / 27720$ & 14 \\
\hline 27.3 & $\{10 \times(1,2), 2 \times(4,9), 3 \times(2,5),(3,8), 7 \times(1,3),(5,18),(1,5)\}$ & 4 & $1 / 360$ & 14 \\
\hline 28 & $\{5 \times(1,2),(5,11),(3,8), 4 \times(1,3),(2,7),(1,5)\}$ & 2 & $23 / 9240$ & 14 \\
\hline 29 & $\{6 \times(1,2),(4,9),(4,11), 3 \times(1,3),(2,7),(1,5)\}$ & 2 & $13 / 3465$ & 14 \\
\hline 29.1 & $\{6 \times(1,2),(4,9),(5,14), 2 \times(1,3),(2,7),(1,5)\}$ & 2 & $1 / 630$ & 14 \\
\hline 30 & $\{7 \times(1,2),(5,11),(3,7),(2,5),(4,11), 5 \times(1,3),(2,7),(1,4),(1,5)\}$ & 3 & $1 / 924$ & 14 \\
\hline 31 & $\{7 \times(1,2),(5,11),(3,7),(2,5),(3,8), 6 \times(1,3),(3,11),(1,5)\}$ & 3 & $1 / 616$ & 14 \\
\hline 32 & $\{8 \times(1,2),(4,9),(3,7),(2,5),(4,11), 5 \times(1,3),(3,11),(1,5)\}$ & 3 & $2 / 693$ & 14 \\
\hline $32 \mathrm{a}$ & $\{8 \times(1,2),(7,16),(2,5),(4,11), 5 \times(1,3),(3,11),(1,5)\}$ & 3 & $1 / 528$ & 14 \\
\hline 33 & $5 \times(1,2), 2 \times(3,7),(3,8),(1,3),(3,10),(2,7)\}$ & 2 & $1 / 840$ & 14 \\
\hline 34 & $\{7 \times(1,2),(4,9),(3,7), 2 \times(2,5),(3,8), 3 \times(1,3), 3 \times(2,7)\}$ & 3 & $1 / 360$ & 14 \\
\hline $34 \mathrm{a}$ & $\{7 \times(1,2),(7,16), 2 \times(2,5),(3,8), 3 \times(1,3), 3 \times(2,7)\}$ & 3 & $1 / 560$ & 14 \\
\hline 35 & $\{5 \times(1,2), 2 \times(3,7),(4,11),(1,3), 2 \times(2,7)\}$ & 2 & $1 / 462$ & 14 \\
\hline 36 & $\{4 \times(1,2),(4,9),(3,7),(2,5), 2 \times(1,3),(3,10),(2,7)\}$ & 2 & $1 / 630$ & 14 \\
\hline $36 \mathrm{a}$ & $\{4 \times(1,2),(7,16),(2,5), 2 \times(1,3),(3,10),(2,7)\}$ & 2 & $1 / 1680$ & 14 \\
\hline $36 \mathrm{~b}$ & $\{4 \times(1,2),(4,9),(3,7),(2,5), 2 \times(1,3),(5,17)\}$ & 2 & $4 / 5355$ & 14 \\
\hline 37 & $6 \times(1,2), 2 \times(4,9), 3 \times(2,5), 4 \times(1,3), 3 \times(2,7)\}$ & 3 & $1 / 315$ & 14 \\
\hline 38 & $\{3 \times(1,2),(5,11),(3,7),(2,5), 3 \times(1,3), 2 \times(2,7)\}$ & 2 & $1 / 770$ & 14 \\
\hline 39 & $\{7 \times(1,2),(4,9),(3,7),(2,5), 2 \times(3,8), 2 \times(1,3),(3,10),(2,7),(1,4)\}$ & 3 & $1 / 630$ & 14 \\
\hline 40 & $\{9 \times(1,2), 2 \times(4,9), 3 \times(2,5), 2 \times(3,8), 4 \times(1,3), 3 \times(2,7),(1,4)\}$ & 4 & $1 / 315$ & 14 \\
\hline 42 & $\{6 \times(1,2),(5,11),(3,7),(2,5), 2 \times(3,8), 3 \times(1,3), 2 \times(2,7),(1,4)\}$ & 3 & $1 / 770$ & 14 \\
\hline 43 & $\{7 \times(1,2),(4,9),(3,7),(2,5),(3,8),(4,11), 2 \times(1,3), 2 \times(2,7),(1,4)\}$ & 3 & $71 / 27720$ & 14 \\
\hline 43.1 & $\{7 \times(1,2),(7,16),(2,5),(3,8),(4,11), 2 \times(1,3), 2 \times(2,7),(1,4)\}$ & 3 & $29 / 18480$ & 14 \\
\hline $43 \mathrm{c}$ & $\{7 \times(1,2),(7,16),(2,5),(7,19), 2 \times(1,3), 2 \times(2,7),(1,4)\}$ & 3 & $31 / 31920$ & 14 \\
\hline 43.2 & $\{7 \times(1,2),(4,9),(3,7),(2,5),(7,19), 2 \times(1,3), 2 \times(2,7),(1,4)\}$ & 3 & $47 / 23940$ & 14 \\
\hline 44 & $\{7 \times(1,2),(4,9),(3,7),(2,5), 2 \times(3,8), 3 \times(1,3),(2,7),(3,11)\}$ & 3 & $43 / 13860$ & 14 \\
\hline 44.1 & $\{7 \times(1,2),(4,9),(3,7),(5,13),(3,8), 3 \times(1,3),(2,7),(3,11)\}$ & 3 & $85 / 72072$ & 14 \\
\hline 44.2 & $\{7 \times(1,2),(4,9),(3,7),(2,5), 2 \times(3,8), 3 \times(1,3),(5,18)\}$ & 3 & $1 / 420$ & 14 \\
\hline 44.3 & $\{7 \times(1,2),(7,16),(2,5), 2 \times(3,8), 3 \times(1,3),(2,7),(3,11)\}$ & 3 & $13 / 6160$ & 14 \\
\hline $44 \mathrm{c}$ & $\{7 \times(1,2),(7,16),(2,5), 2 \times(3,8), 3 \times(1,3),(5,18)\}$ & 3 & $1 / 720$ & 14 \\
\hline 45 & $\{3 \times(1,2), 2 \times(4,9),(3,8), 3 \times(1,3),(2,7),(1,4)\}$ & 2 & $1 / 504$ & 14 \\
\hline 46 & $\{6 \times(1,2), 2 \times(4,9), 2 \times(2,5),(3,8), 3 \times(1,3),(3,10),(2,7),(1,4)\}$ & 3 & $1 / 504$ & 14 \\
\hline $46 \mathrm{~b}$ & $\{6 \times(1,2), 2 \times(4,9), 2 \times(2,5),(3,8), 3 \times(1,3),(5,17),(1,4)\}$ & 3 & $7 / 6120$ & 14 \\
\hline 48 & $\{4 \times(1,2),(4,9),(3,7),(4,11),(1,3),(3,10),(1,4)\}$ & 2 & $19 / 13860$ & 14 \\
\hline 49 & $\{5 \times(1,2),(5,11),(4,9), 2 \times(2,5),(3,8), 4 \times(1,3), 2 \times(2,7),(1,4)\}$ & 3 & $47 / 27720$ & 14 \\
\hline $49 \mathrm{a}$ & $\{(5 \times(1,2),(9,20), 2 \times(2,5),(3,8), 4 \times(1,3), 2 \times(2,7),(1,4)\}$ & 3 & $1 / 840$ & 14 \\
\hline 50 & $\{6 \times(1,2), 2 \times(2,9), 2 \times(2,5),(4,11), 3 \times(1,3), 2 \times(2,7),(1,4)\}$ & 3 & $41 / 13860$ & 14 \\
\hline 51 & $\{6 \times(1,2), 2 \times(4,9), 2 \times(2,5),(3,8), 4 \times(1,3),(2,7),(3,11)\}$ & 3 & $97 / 27720$ & 14 \\
\hline 51.1 & $\{6 \times(1,2), 2 \times(4,9),(2,5),(5,13), 4 \times(1,3),(2,7),(3,11)\}$ & 3 & $71 / 45045$ & 14 \\
\hline 52 & $\{4 \times(1,2),(3,7), 2 \times(2,5), 2 \times(3,8), 2 \times(1,3),(1,5)\}$ & 2 & $1 / 420$ & 14 \\
\hline 53 & $3 \times(1,2),(4,9), 3 \times(2,5),(3,8), 3 \times(1,3),(1,5)\}$ & 2 & $1 / 360$ & 14 \\
\hline 54 & $\{2 \times(1,2), 2 \times(3,7), 3 \times(2,5),(3,8),(1,3),(2,7)\}$ & 2 & $1 / 840$ & 14 \\
\hline
\end{tabular}




\begin{tabular}{|l|l|l|l|l|}
\hline 56 & $\{(1,2),(4,9),(3,7), 4 \times(2,5), 2 \times(1,3),(2,7)\}$ & 2 & $1 / 630$ & 14 \\
\hline 58 & $\{4 \times(1,2),(4,9),(3,7), 4 \times(2,5), 2 \times(3,8), 2 \times(1,3),(2,7),(1,4)\}$ & 3 & $1 / 630$ & 14 \\
\hline 59 & $\{2 \times(1,2), 2 \times(3,7), 2 \times(2,5),(3,8),(4,11),(1,4)\}$ & 2 & $3 / 3080$ & 14 \\
\hline 60 & $3 \times(1,2), 2 \times(4,9), 5(2,5),(3,8), 3 \times(1,3),(2,7),(1,4)\}$ & 3 & $1 / 504$ & 14 \\
\hline 62 & $\{(1,2),(4,9),(3,7), 3 \times(2,5),(4,11),(1,3),(1,4)\}$ & 2 & $19 / 13860$ & 14 \\
\hline
\end{tabular}

Table II-1

\begin{tabular}{|l|l|l|l|l|}
\hline No. & $B^{0}(X)$ & $K_{X}^{3}$ & $\chi$ & $\left(P_{3}, P_{4}, P_{5}, P_{6}\right)$ \\
\hline 1 & $\{5 *(1,2), 2 *(1,3)\}$ & $1 / 6$ & 0 & $(3,5,7,11)$ \\
\hline 2 & $\{5 *(1,2),(1,3),(1,4)\}$ & $1 / 12$ & 0 & $(3,5,6,9)$ \\
\hline 3 & $\{18 *(1,2),(1,3)\}$, & $1 / 3$ & 1 & $(1,5,6,13)$ \\
\hline 4 & $\{(18-4 t) *(1,2), 3 t *(1,3),(1,4)\}, t=0,1,2$ & $1 / 4$ & 1 & $(1+t, 5,5+t, 11+t)$ \\
\hline 5 & $\{(18-4 t) *(1,2), 3 t *(1,3),(1,5)\}, 5 \leq r \leq 12 ; t=0,1,2$ & $1 / \mathrm{r}$ & 1 & $(1+t, 5,5+t, 10+t)$ \\
\hline 6 & $\{(17-4 t) *(1,2),(2+3 t) *(1,3)\}, t=0,1,2$ & $1 / 6$ & 1 & $(1+t, 4,4+t, 9+t)$ \\
\hline 7 & $\{(14-4 t) *(1,2),(2+3 t) *(1,3), 2 *(1,4)\}, t=0,1$ & $1 / 6$ & 1 & $(2+t, 5,5+t, 10+t)$ \\
\hline 8 & $\{(14-4 t) *(1,2),(2+3 t) *(1,3),(1,4),(1,5)\}, t=0,1$ & $7 / 60$ & 1 & $(2+t, 5,5+t, 9+t)$ \\
\hline 9 & $\{(14-4 t) *(1,2),(2+3 t) *(1,3),(1,4),(1,6)\}, t=0,1$ & $1 / 12$ & 1 & $(2+t, 5,5+t, 9+t)$ \\
\hline 10 & $\{(14-4 t) *(1,2),(1+3 t) *(1,3), 3 *(1,4)\}, t=0,1$ & $1 / 12$ & 1 & $(2+t, 5,4+t, 8+t)$ \\
\hline 11 & $\{(17-4 t) *(1,2),(1+3 t) *(1,3),(1,4)\}, t=0,1,2$ & $1 / 12$ & 1 & $(1+t, 4,3+t, 7+t)$ \\
\hline
\end{tabular}

Table II-2

\begin{tabular}{|l|l|l|l|l|}
\hline No. & $B^{0}(X)$ & $K_{X}^{3}$ & $\chi$ & $\left(P_{3}, P_{4}, P_{5}, P_{6}\right)$ \\
\hline 1 & $\{27 *(1,2), 2 *(1,3),(1, r)\}$ & $\frac{1}{6}+\frac{1}{r}$ & 2 & $(0,5,5,13)$ \\
\hline 2 & $\begin{array}{l}\{(27-4 t) *(1,2),(1+3 t) *(1,3), \\
2 *(1,4)\}, t=0,1 .\end{array}$ & $1 / 3$ & 2 & $(t, 5,4+t, 12+t)$ \\
\hline 3 & $\begin{array}{l}\{(27-4 t) *(1,2),(1+3 t) *(1,3), \\
(1,4),(1, r)\}, 5 \leq r ; t=0,1,2 .\end{array}$ & $\frac{1}{12}+\frac{1}{r}$ & 2 & $(t, 5,4+t, 11+t)$ \\
\hline 4 & $\begin{array}{l}\{(27-4 t) *(1,2),(1+3 t) *(1,3), \\
\left.\left(1, r_{1}\right),\left(1, r_{2}\right)\right\},\left(r_{1}, r_{2}\right) \in I_{4} ; t=0,1,2,3 .\end{array}$ & $\frac{1}{r_{1}}+\frac{1}{r_{2}}-\frac{1}{6}$ & 2 & $(t, 5,4+t, 10+t)$ \\
\hline 5 & $\{(26-4 t) *(1,2),(4+3 t) *(1,3)\}, t=0,1$. & $1 / 3$ & 2 & $(t, 4,4+t, 12+t)$ \\
\hline 6 & $\begin{array}{l}\{(27-4 t) *(1,2), 3 t *(1,3), 3 *(1,4)\}, \\
t=0,1,2,3 .\end{array}$ & $1 / 4$ & 2 & $(t, 5,3+t, 10+t)$ \\
\hline 7 & $\begin{array}{l}\{(27-4 t) *(1,2), 3 t *(1,3), 2 *(1,4), \\
(1, r)\}, 5 \leq r \leq 12 ; t=0,1,2,3 .\end{array}$ & $1 / \mathrm{r}$ & 2 & $(t, 5,3+t, 9+t)$ \\
\hline 8 & $\begin{array}{l}\left\{(27-4 t) *(1,2), 3 t *(1,3),(1,4),\left(1, r_{1}\right),\right. \\
\left.\left(1, r_{2}\right)\right\},\left(r_{1}, r_{2}\right) \in I_{3} ; t=0,1,2,3 .\end{array}$ & $\frac{1}{r_{1}}+\frac{1}{r_{2}}-\frac{1}{4}$ & 2 & $(t, 5,3+t, 8+t)$ \\
\hline 9 & $\begin{array}{l}\{(27-4 t) *(1,2), 3 t *(1,3), 3 *(1,5)\}, \\
t=0,1,2,3 .\end{array}$ & $1 / 10$ & 2 & $(t, 5,3+t, 7+t)$ \\
\hline 10 & $\begin{array}{l}\{(26-4 t) *(1,2),(3+3 t) *(1,3),(1,4)\}, \\
t=0,1,2,3 .\end{array}$ & $1 / 4$ & 2 & $(0,4,3+t, 10+t)$ \\
\hline 11 & $\begin{array}{l}\{(26-4 t) *(1,2),(3+3 t) *(1,3),(1, r)\}, \\
5 \leq r \leq 12 ; t=0,1,2,3 .\end{array}$ & $1 / \mathrm{r}$ & 2 & $(0,4,3+t, 9+t)$ \\
\hline 12 & $\{(25-4 t) *(1,2),(5+3 t) *(1,3)\}, t=0,1,2,3$. & $1 / 6$ & 2 & $(t, 3,2+t, 8+t)$ \\
\hline 13 & $\begin{array}{l}\{(26-4 t) *(1,2),(2+3 t) *(1,3), 2 *(1,4)\}, \\
t=0,1,2,3 .\end{array}$ & $1 / 6$ & 2 & $(t, 4,2+t, 8+t)$ \\
\hline 14 & $\begin{array}{l}\{(26-4 t) *(1,2),(2+3 t) *(1,3),(1,4),(1,5)\}, \\
t=0,1,2,3 .\end{array}$ & $7 / 60$ & 2 & $(t, 4,2+t, 7+t)$ \\
\hline 15 & $\begin{array}{l}\{(26-4 t) *(1,2),(2+3 t) *(1,3),(1,4), \\
(1,6)\}, t=0,1,2,3 .\end{array}$ & $1 / 12$ & 2 & $(t, 4,2+t, 7+t)$ \\
\hline 16 & $\begin{array}{l}\{(25-4 t) *(1,2),(4+3 t) *(1,3),(1,4)\}, \\
t=0,1,2,3 .\end{array}$ & $1 / 12$ & 2 & $(t, 3,1+t, 6+t)$ \\
\hline 17 & $\begin{array}{l}\{(26-4 t) *(1,2),(1+3 t) *(1,3), 3 *(1,4)\}, \\
t=0,1,2,3 .\end{array}$ & $1 / 12$ & $2,4,1+t, 6+t)$ \\
\hline
\end{tabular}

where

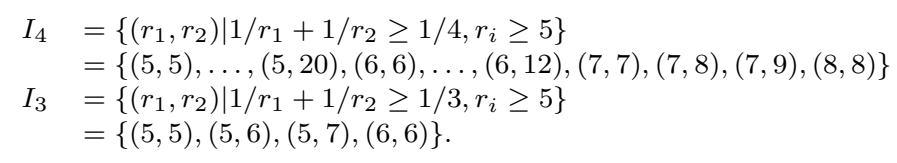


Table II-3

\begin{tabular}{|c|c|c|c|c|}
\hline & $B^{0}(X)$ & $K_{X}^{3}$ & $\bar{x}$ & $\left(P_{3}, P_{4}, P_{5}, P_{6}\right)$ \\
\hline 1 & $\{32 *(1,2), 5 *(1,3), 2 *(1,4),(1, r)\}, 5 \leq r$ & $\frac{1}{6}+\frac{1}{r}$ & 3 & $(0,5,4,13)$ \\
\hline 2 & $\begin{array}{l}\{(32-4 t) *(1,2),(5+3 t) *(1,3),(1,4) \\
\left.\left(1, r_{1}\right),\left(1, r_{2}\right)\right\},\left(r_{1}, r_{2}\right) \in I_{6}, t \leq 1\end{array}$ & $\frac{1}{r_{1}}+\frac{1}{r_{2}}-\frac{1}{12}$ & 3 & $(t, 5,4+t, 12+t)$ \\
\hline 3 & $\begin{array}{l}\left\{(32-4 t) *(1,2),(5+3 t) *(1,3),\left(1, r_{1}\right)\right. \\
\left.\left(1, r_{2}\right),\left(1, r_{3}\right)\right\},\left(r_{1}, r_{2}, r_{3}\right) \in J, t \leq 2\end{array}$ & $\frac{1}{r_{1}}+\frac{1}{r_{2}}+\frac{1}{r_{3}}-\frac{1}{3}$ & 3 & $(t, 5,4+t, 11+t)$ \\
\hline 4 & $\begin{array}{l}\{(31-4 t) *(1,2),(7+3 t) *(1,3) \\
2 *(1,4)\}, t \leq 1\end{array}$ & $1 / 3$ & 3 & $(t, 4,3+t, 12+t)$ \\
\hline 5 & $\begin{array}{l}\{(31-4 t) *(1,2),(7+3 t) *(1,3) \\
(1,4),(1, r)\}, 5 \leq r ; t \leq 2\end{array}$ & $\frac{1}{12}+\frac{1}{r}$ & 3 & $(t, 4,3+t, 11+t)$ \\
\hline 6 & $\begin{array}{l}\{(31-4 t) *(1,2),(7+3 t) *(1,3) \\
\left.\left(1, r_{1}\right),\left(1, r_{2}\right)\right\},\left(r_{1}, r_{2}\right) \in I_{4} ; t \leq 3\end{array}$ & $\frac{1}{r_{1}}+\frac{1}{r_{2}}-\frac{1}{6}$ & 3 & $(t, 4,3+t, 10+t)$ \\
\hline 7 & $\{(30-4 t) *(1,2),(10+3 t) *(1,3)\}, t=0,1$. & $1 / 3$ & 3 & $(t, 3,3+t, 12+t)$ \\
\hline 8 & $\begin{array}{l}\{(31-4 t) *(1,2),(6+3 t) *(1,3) \\
3 *(1,4)\}, t=0,1,2,3\end{array}$ & $1 / 4$ & 3 & $(t, 4,2+t, 10+t)$ \\
\hline 9 & $\begin{array}{l}\{(31-4 t) *(1,2),(6+3 t) *(1,3) \\
2 *(1,4),(1, r)\}, 5 \leq r \leq 12 ; t=0,1,2,3\end{array}$ & $1 / \mathrm{r}$ & 3 & $(t, 4,2+t, 9+t)$ \\
\hline 10 & $\begin{array}{l}\{(31-4 t) *(1,2),(6+3 t) *(1,3) \\
\left.(1,4),\left(1, r_{1}\right),\left(1, r_{2}\right)\right\},\left(r_{1}, r_{2}\right) \in I_{3} ; t \leq 3\end{array}$ & $\frac{1}{r_{1}}+\frac{1}{r_{2}}-\frac{1}{4}$ & 3 & $(t, 4,2+t, 8+t)$ \\
\hline 11 & $\begin{array}{l}\{(31-4 t) *(1,2),(6+3 t) *(1,3), \\
3 *(1,5)\}, t=0,1,2,3\end{array}$ & $1 / 10$ & 3 & $(t, 4,2+t, 7+t)$ \\
\hline 12 & $\begin{array}{l}\{(30-4 t) *(1,2),(9+3 t) *(1,3), \\
(1,4)\}, t=0,1,2,3\end{array}$ & $1 / 4$ & 3 & $(0,3,2+t, 10+t)$ \\
\hline 13 & $\begin{array}{l}\{(30-4 t) *(1,2),(9+3 t) *(1,3) \\
(1, r)\}, 5 \leq r \leq 12 ; t=0,1,2,3\end{array}$ & $1 / \mathrm{r}$ & 3 & $(0,3,2+t, 9+t)$ \\
\hline 14 & $\begin{array}{l}\{(30-4 t) *(1,2),(8+3 t) *(1,3), \\
2 *(1,4)\}, t=0,1,2,3\end{array}$ & $1 / 6$ & 3 & $(t, 3,1+t, 8+t)$ \\
\hline 15 & $\begin{array}{l}\{(30-4 t) *(1,2),(8+3 t) *(1,3), \\
(1,4),(1,5)\}, t=0,1,2,3\end{array}$ & $7 / 60$ & 3 & $(t, 3,1+t, 7+t)$ \\
\hline 16 & $\begin{array}{l}\{(30-4 t) *(1,2),(8+3 t) *(1,3), \\
(1,4),(1,6)\}, t=0,1,2,3\end{array}$ & $1 / 12$ & 3 & $(t, 3,1+t, 7+t)$ \\
\hline 17 & $\begin{array}{l}\{(30-4 t) *(1,2),(7+3 t) *(1,3), \\
3 *(1,4)\}, t=0,1,2,3\end{array}$ & $1 / 12$ & 3 & $(t, 3, t, 6+t)$ \\
\hline
\end{tabular}

where

$I_{4}=\left\{\left(r_{1}, r_{2}\right) \mid 1 / r_{1}+1 / r_{2} \geq 1 / 4, r_{i} \geq 5\right\}$

$=\{(5,5), \ldots,(5,20),(6,6), \ldots,(6,12),(7,7),(7,8),(7,9),(8,8)\}$

$I_{3}=\left\{\left(r_{1}, r_{2}\right) \mid 1 / r_{1}+1 / r_{2} \geq 1 / 3, r_{i} \geq 5\right\}$

$=\{(5,5),(5,6),(5,7),(6,6)\}$.

$I_{6}=\left\{\left(r_{1}, r_{2}\right) \mid 1 / r_{1}+1 / r_{2} \geq 1 / 6, r_{i} \geq 5\right\}$

$=\left\{\left(5, s_{5}\right),\left(6, s_{6}\right),\left(7, s_{7}\right),\left(8, s_{8}\right),\left(9, s_{9}\right),\left(10, s_{10}\right),(11,11),(11,12),(11,13),(12,12)\right\}$,

$5 \leq s_{1}, 6 \leq s_{2}, 7 \leq s_{7} \leq 42,8 \leq s_{8} \leq 24,9 \leq s_{9} \leq 18,10 \leq s_{10} \leq 15$.

$J=\left\{\left(r_{1}, r_{2}, r_{3}\right) \mid 1 / r_{1}+1 / r_{2}+1 / r_{3} \geq 5 / 12, r_{i} \geq 5\right\}$

$=\left\{\left(5,5, s_{1}\right),\left(5,6, s_{2}\right),\left(5,7, s_{3}\right),(5,8,8),(5,8,9),(5,8,10),(5,9,9),\left(6,6, s_{4}\right),(6,7,7),(6,7,8)\right.$,

$(6,7,9),(6,8,8),(7,7,7)\}, 5 \leq s_{1} \leq 60,6 \leq s_{2} \leq 20,7 \leq s_{3} \leq 13,6 \leq s_{4} \leq 12$.

\section{REFERENCES}

[1] W. Barth, C. Peters, A. Van de Ven, Compact complex surfaces, SpringerVerlag, 1984.

[2] C. Birkar, P. Cascini, C. D. Hacon, J. McKernan, Existence of minimal models for varieties of log general type, J. Amer. Math. Soc. 23 (2010), no. 2, 405-468.

[3] E. Bombieri, Canonical models of surfaces of general type, Inst. Hautes Études Sci. Publ. Math. 42 (1973), 171-219.

[4] F. Catanese, R. Pignatelli, Fibrations of low genus. I. Ann. Sci. École Norm. Sup. (4) 39 (2006), no. 6, 1011-1049. 
[5] J. A. Chen, M. Chen, The canonical volume of 3-folds of general type with $\chi \leq 0$, J. Lond. Math. Soc. (2) 78 (2008), no. 3, 693-706.

[6] J. A. Chen, M. Chen, Explicit birational geometry of threefolds of general type, I, Ann. Sci. Éc. Norm. Supér (43) 2010, 365-394.

[7] J. A. Chen, M. Chen, Explicit birational geometry of threefolds of general type, II. J. of Diff. Geom. 86 (2010), 237-271.

[8] J. A. Chen, M. Chen, Z. Jiang, On 6-canonical map of irregular threefolds of general type, Math. Res. Lett. 20 (2013), 33-39.

[9] J. A. Chen, C. D. Hacon, Pluricanonical systems on irregular 3-folds of general type. Math. Z. 255(2007), no. 2, 343-355.

[10] M. Chen, Canonical stability of 3-folds of general type with $p_{g} \geq 3$, Internat. J. Math. 14 (2003), no. 5, 515-528.

[11] M. Chen, A sharp lower bound for the canonical volume of 3-folds of general type, Math. Ann. 337 (2007), no. 4, 887-908.

[12] M. Chen, Some birationality criteria on 3-folds with $p_{g}>1$, arXiv: 1111.6513.

[13] M. Chen, K. Zuo, Complex projective 3-fold with non-negative canonical EulerPoincare characteristc, Commun. Anal. Geom. 16 (2008), 159-182.

[14] M. Chen, On pluricanonical systems of algebraic varieties of general type. Algebraic geometry in East Asia - Seoul 2008, 215-236, Adv. Stud. Pure Math., 60, Math. Soc. Japan, Tokyo, 2010.

[15] A. R. Iano-Fletcher, Working with weighted complete intersections. Explicit birational geometry of 3-folds, 101-173, London Math. Soc. Lecture Note Ser., 281, Cambridge Univ. Press, Cambridge, 2000.

[16] T. Fujita, On Kähler fiber spaces over curves, J. Math. Soc. Japan 30 (1978), no. $4,779-794$

[17] C. D. Hacon, J. McKernan, Boundedness of pluricanonical maps of varieties of general type, Invent. Math. 166 (2006), no. 1, 1-25.

[18] C. D. Hacon, J. McKernan, Boundedness of pluricanonical maps of varieties of general type, In Proceedings of the International Congress of Mathematicians, Volume II, pages 427-449, New Delhi, 2010. Hindustan Book Agency.

[19] Y. Kawamata, A generalization of Kodaira-Ramanujam's vanishing theorem, Math. Ann. 261 (1982), 43-46.

[20] Y. Kawamata, On the extension problem of pluricanonical forms. Algebraic geometry: Hirzebruch 70 (Warsaw, 1998), 193-207, Contemp. Math., 241, Amer. Math. Soc., Providence, RI, 1999.

[21] Y. Kawamata, Flops connect minimal models, Publ. Res. Inst. Math. Sci. 44 (2008), no. 2, 419-423.

[22] Y. Kawamata, K. Matsuda, K. Matsuki, Introduction to the minimal model problem, Adv. Stud. Pure Math. 10(1987), 283-360.

[23] J. Kollár, Flops, Nagoya Math. Jour. 113 (1989), 15-36.

[24] J. Kollár, S. Mori, Birational geometry of algebraic varieties, 1998, Cambridge Univ. Press.

[25] V. Maşek, Very ampleness of adjoint linear systems on smooth surfaces with boundary, Nagoya Math. J. 153 (1999), 1-29.

[26] Y. Miyaoka, Tricanonical maps of numerical Godeaux surfaces, Invent. Math. 34 (1976), no. 2, 99-111.

[27] M. Reid, Surfaces with $p_{g}=0, K^{2}=1$. J. Fac. Sci. Univ. Tokyo Sect. IA Math. 25 (1978), no. 1, 75-92.

[28] M. Reid, Chapters on algebraic surfaces. Complex algebraic geometry (Park City, UT, 1993), 3-159, IAS/Park City Math. Ser., 3, Amer. Math. Soc., Providence, RI, 1997. 
[29] M. Reid, Young person's guide to canonical singularities. Algebraic geometry, Bowdoin, 1985 (Brunswick, Maine, 1985), 345-414, Proc. Sympos. Pure Math., 46, Part 1, Amer. Math. Soc., Providence, RI, 1987.

[30] Y. T. Siu, Finite generation of canonical ring by analytic method, Sci. China Ser. A 51 (2008), no. 4, 481-502.

[31] S. Takayama, Pluricanonical systems on algebraic varieties of general type. Invent. Math. 165 (2006), no. 3, 551-587.

[32] H. Tsuji, Pluricanonical systems of projective varieties of general type. I. Osaka J. Math. 43 (2006), no. 4, 967-995.

[33] E. Viehweg, Vanishing theorems, J. reine angew. Math. 335 (1982), 1-8.

National Center for Theoretical Sciences, Taipei Office, and Department of Mathematics, National Taiwan University, Taipei, 106, Taiwan

E-mail address: jkchen@math.ntu.edu.tw

Institute of Mathematics \& LMNS, Fudan University, Shanghai, 200433, People's Republic of China

E-mail address: mchen@fudan.edu.cn 